FHWA/IN/JTRP-2002/19

Final Report

IMPROVING EFFICIENCY OF INDOT TRAFFIC

DATA COLLECTION USING GPS DEVICES

Shuo Li,

Karen Zhu

B. H. W. van Gelder

John Nagle

Carl Tuttle

June 2002 
FINAL REPORT

FHWA/IN/JTRP-2002/19

\title{
IMPROVING EFFICIENCY OF INDOT TRAFFIC DATA COLLECTION USING GPS DEVICES
}

\author{
By \\ Shuo $\mathrm{Li}$ \\ Traffic Safety Research Engineer \\ Research Division \\ Indiana Department of Transportation \\ Karen Zhu \\ Systems Analyst \\ Research Division \\ Indiana Department of Transportation \\ B. H. W. van Gelder \\ Professor \\ School of Civil Engineering \\ Purdue University \\ John Nagle \\ Safety/Congestion Management Engineer \\ Program Development Division \\ Indiana Department of Transportation \\ Carl Tuttle \\ Field Engineer \\ Operations Support Division \\ Indiana Department of Transportation \\ Joint Highway Research Program \\ Project No. C-36-17CCC \\ File No. 8-4-55 \\ SPR-2392 \\ Prepared in Cooperation with the \\ Indiana Department of Transportation \\ and the \\ U.S. Department of Transportation \\ Federal Highway Administration
}

The contents of this report reflect the views of the authors who are responsible for the facts and the accuracy of the data presented herein. The contents do not necessarily reflect the official views or policies of the Federal Highway Administration and the Indiana Department of Transportation. The report does not constitute a standard, specification, or regulation.

Purdue University

West Lafayette, IN 47907

June 2002 
TECHNICAL REPORT STANDARD TITLE PAGE

\begin{tabular}{|l|l|l|}
\hline 1. Report No. & 2. Government Accession No. & \\
FHWA/IN/JTRP-2002/19 & & . \\
\hline
\end{tabular}

\section{Title and Subtitle}

Improving Efficiency of INDOT Traffic Data Collection Using GPS Devices

\section{Author(s)}

Shuo Li, Karen Zhu, B. H. W. van Gelder, John Nagle, and Carl Tuttle

9. Performing Organization Name and Address

Joint Transportation Research Program

1284 Civil Engineering Building

Purdue University

West Lafayette, IN 47907-1284

12. Sponsoring Agency Name and Address

Indiana Department of Transportation

Division of Research

1205 Montgomery Street

West Lafayette, IN 47906

3. Recipient Catalog No.

5. Report Date

June 2002

6. Performing Organization Code

8. Performing Organization Report No.

FHWA/IN/JTRP-2002/19

10. Work Unit No.

11. Contract or Grant No.

SPR-2392

13. Type of Report and Period Covered

Final Report

\section{Sponsoring Agency Code}

\section{Supplementary Notes}

Prepared in cooperation with the Indiana Department of Transportation and Federal Highway Administration.

\section{Abstract}

INDOT has utilized many new technologies to reduce travel delay, mitigate traffic congestion, and enhance public and employees' safety. Many projects are underway, including re-timing of most existing traffic signals and pavement maintenance and rehabilitation. To evaluate signal system performance, prioritize alternatives of improvement, conduct before and after studies and investigate work zone safety, it requires a large amount of traffic data such as travel time, speed and delay. Issues, such as safety, efficiency and cost, may arise associated with the current methods. For the current manual or automatic data collection, it usually requires two operators, a driver and a recorder. The current methods also require much time for data processing and the resulting data files may not be reusable. With the global positioning system (GPS) technologies, it appears that traffic data collection can be performed more safely and efficiently.

The objective of this study is to investigate use of the GPS technologies to improve efficiency of INDOT traffic data collection, to enhance field operation safety, and to reduce potential human errors. Primary emphasis is given to utilization of GPS receivers to acquire traffic data so as to generate a reusable data file. This kind of data files is essential for providing INDOT traffic engineers with consistent information for assessing the performance of signal systems. Secondary emphasis is given to use of the GPS data in specific transportation studies, such as travel time and delay studies, work zone studies, and congestion management.

In this study, the GPS techniques were examined and the Trimble AgGPS 132 devices were tested using the precisely known geographic points. A computer program, GPS-Trek, was developed for data collection and data processing. A huge amount of data was collected over the selected routes. Based on the results of analysis and field tests, a summary of the major findings are presented below:

The proposed GPS data collection system is inexpensive and cost-effective. It can improve the efficiency of traffic data collection, save manpower and enhance field operation safety. The AgGPS 132 receiver can provide traffic data of high accuracy and consistency. The field data may exhibit gaps in "deep" urban canyons. However, through interpolation and proper data screening these disadvantages may be limited. The removal of Selective Availability from the GPS signals further improves the accuracy of GPS data. It was shown that the relative positional accuracy using a DGPS service is well below the 0.5 meter level.

The GPS-Trek consists of two components, one for data collection and the other for data processing. The program is free of personal interpretation during data collection and provides a consistent system of analysis. The resulting data files are reusable. The data file can be easily exported to Microsoft ${ }^{\circledR}$ Excel, allowing traffic engineers to utilize their own experience and judgment for data analysis and specific transportation studies.

A modified equation is presented to estimate the sample size requirements for field data collection using GPS devices. It was shown that in the filed tests, the modified equation produced a realistic estimate of the minimum sample size. Generally, a minimum of three initial test runs should be performed. If possible, five initial test runs for $90 \%$ confidence or six for $95 \%$ confidence are required to reduce the potential errors. The proposed system can be readily used for various transportation studies. This system bases its procedures on the existing INDOT highway digital map, resulting in a great saving in terms of manpower and time for creating a high resolution base map. The system also allows us to mark any critical points during data collection or data processing. As a result, it is possible for us to provide graphical reports, such as vehicle trajectory and speed profile that are required in the Manual of Transportation Engineering Studies and get a full picture of traveling situation on the test route. Also, this system can be used for measuring congestion on a system level.

\section{Key Words}

\section{Distribution Statement}

GPS, GPS-Trek, travel time, travel speed, travel delay, efficiency, safety, work zone, congestion

No Restrictions. This document is available to the public through the National Technical Information Service, Springfield, VA 22161

\begin{tabular}{|c|c|c|c|}
\hline 19. Security Classif. (of this report) & 20. Security Classif. (of this Page) & 21. No. of Pages & 22. Price \\
Unclassified & Unclassified & 92 & \\
\hline
\end{tabular}




\section{TECHNICAL Summary}

INDOT Research

Technology Transfer and Project Implementation Information

TRB Subject Code: 55-03 Traffic Flow Capacity and Measurements

June 2002

Publication No.: FHWA/IN/JTRP-2002/19

Final Report

\section{Improving Efficiency of INDOT Traffic Data Collection Using GPS Devices}

\section{Introduction}

INDOT has utilized many new technologies to reduce travel delay, mitigate traffic congestion, and enhance public and employees' safety. Many projects are underway, including re-timing of most existing traffic signals and pavement maintenance and rehabilitation. INDOT engineers are frequently required to collect traffic data such as travel time, travel speed, and delay in order to evaluate system performance, prioritize alternatives of improvement, and conduct before and after studies.

Issues, such as safety, efficiency and cost, may arise associated with the current data collection methods. For the current manual or automatic data collection, it usually requires two operators, a driver and a recorder. The current methods also require much time for data processing and the resulting data files may not be reusable. With the global positioning system
(GPS) technologies, it appears that traffic data collection can be performed more safely and efficiently.

The objective of this study is to investigate the use of the GPS technologies to improve efficiency of INDOT traffic data collection, to enhance field operation safety and to reduce potential human errors. Primary emphasis is given to utilization of GPS receivers to acquire the traffic data so as to generate a reusable data file. This kind of data files is essential for providing INDOT traffic engineers with consistent information for assessing the performance of signal systems. Secondary emphasis is given to use of the GPS data files in specific transportation studies, such as travel time and delay studies, work zone studies and congestion management.

\section{Findings}

In this study, the GPS technique was examined and a specific GPS device, $\operatorname{AgGPS}^{\mathrm{TM}} 132$ device was tested using the precisely known geographic points as references. A computer program, GPSTrek, was developed for data collection and data processing. A huge amount of data was collected over the selected routes. Based on the results of analysis and field tests, a summary of the major findings are presented below:

The proposed GPS data collection system is inexpensive and cost-effective. It can improve the efficiency of traffic data collection, save manpower and enhance field operation safety. The $\mathrm{AgGPS}^{\mathrm{TM}} 132$ receiver can provide traffic data of high accuracy and consistency. The field data may exhibit gaps in "deep" urban

canyons. However, through interpolation and proper data screening for spurious effects these disadvantages may be limited. The removal of Selective Availability from the GPS signals considerably lessened the need for DGPS services. It was shown that the relative positional accuracy using a DGPS service is well below the 0.5 meter level.

The GPS-Trek consists of two components, one for data collection and the other for data processing. The program is free of personal interpretation during data collection and provides a consistent system of analysis. The data saved in the resulting files are versatile and reusable. The format of the data file is specially designed so that the data can be handled 
efficiently. The data file can also be easily exported to Micrsoft $^{\circledR}$ Excel, allowing traffic engineers to utilize their own experience and judgment for data analysis and specific transportation studies.

A modified equation is presented to estimate the sample size requirements for field data collection. It is shown that in the filed tests, the modified equation can produce a realistic estimate of the minimum sample size. Generally, a minimum of three initial test runs should be performed. If possible, five initial test runs for $90 \%$ confidence or six for $95 \%$ confidence are required to reduce the potential errors.

\section{Implementation}

The SPR-2392 research group proposes that the system developed in this study be considered as a standard method for INDOT traffic data collection and analysis. Workshops will be conducted to provide instruction for initializing and operating the system, running the GPS-Trek program, creating worksheets and generating graphical reports. Brainstorming sessions will be scheduled to explore use of the system and the highly accurate and dense data in highway inventory system.

Trials conducted during this phase of the study reveal that the analysis will be greatly enhanced if a state-wide traffic signal data base is being integrated as a layer under the existing
The proposed system can be readily used for various transportation studies. It bases its procedures on the existing INDOT highway digital map, resulting in a great saving in terms of manpower and time by avoiding the need to create a high resolution base map. The system also allows us to mark land marks during data collection or data processing. As a result, it is possible for us to provide graphical reports, such as vehicle trajectory and speed profile that are required in the Manual of Transportation Engineering Studies and get a full picture of traffic performance on the test route.

\section{Contacts}

For more information:

Dr. Shou Li

Principal Investigator

Indiana Department of Transportation

Division of Research

P.O. Box 2279

West Lafayette, IN 47906

Phone: (765) 463-1521

Fax: (765) 497-1665

\section{Dr. Karen Zhu}

Co-Principal Investigator

Indiana Department of Transportation

Division of Research

P.O. Box 2279

West Lafayette, IN 47906

Phone: (765) 463-1521

Fax: (765) 497-1665 base map. The by-product of this research is an improved state route database. Further studies are needed so as to reveal how to imbed the traffic positional data into the state route data base on a regular basis and address issues at the management level.

It is proposed that this feasibility study lead to possible implementation and new test trials to be conducted in one or more INDOT districts. Experience gathered during these trials will lead to improved and more detailed manuals of operation of the GPS data collection system.
Prof. B.H.W. van Gelder

Co-Principal Investigator

School of Civil Engineering

Purdue University

West Lafayette, IN 47907-1284

Phone: (765) 494-2165

Fax: (765) 496-1105

Purdue University

Joint Transportation Research Program

School of Civil Engineering

West Lafayette, IN 47907-1284

Phone: (765) 494-9310

Fax: (765) 496-1105 


\section{TABLE OF CONTENTS}

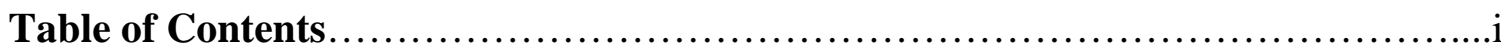

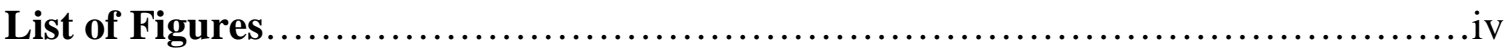

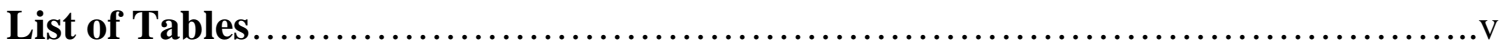

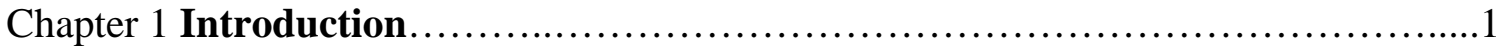

1.1 Problem Statement and Research Objective ............................. .

1.2 Research Scope and Approach........................................... 3

1.3 Proposed Hardware and Software for the System.........................

GPS Devices.............................................................

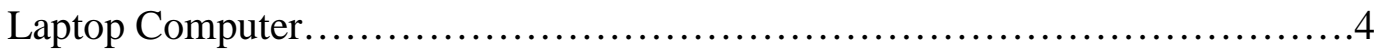

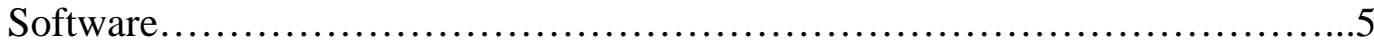

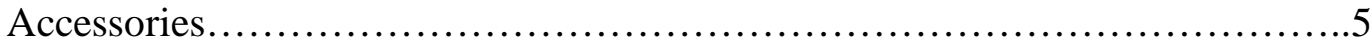

Chapter 2 Selection of GPS Devices for Traffic Data Collection......................6

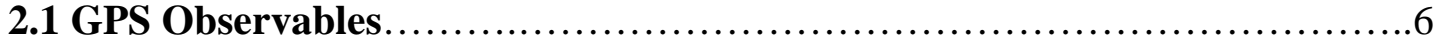

Coded Signals on a Carrier Wave ......................................... 6

Phase of the Carrier Wave Signal................................................ 7

2.2 Differential GPS or Single Point Positioning ............................. 8

Differential Techniques....................................................... 8

Single Point Positioning ...........................................................

2.3 Selection GPS Receivers and DGPS Service.............................10

Chapter 3 AgGPS ${ }^{\mathrm{TM}} 132$ Validation Tests...........................................12

3.1 Validation Tests before May 1, 2002 (SA on, OmniStar off).................12

First Data: April 2000................................................. 12

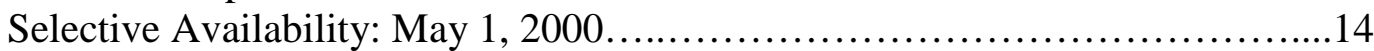

Smoothing Filter: May 1, 2000..........................................15

3.2 Validation Tests on May 1, 2002 (SA on, OmniStar on) .....................16

Data while OmniSTAR Service Being Enabled: May 1, 2000..................16

Data after Enabling OmniSTAR Service: May 1, 2000.........................17

3.3 Validation Tests on May 2, 2002 (SA off, OmniStar on/off) ..................19

SA off, while OmniSTAR Service Being Enabled: May 2, 2000.................19

SA off, while OmniSTAR Service Being Disabled: May 2, 2000...................20

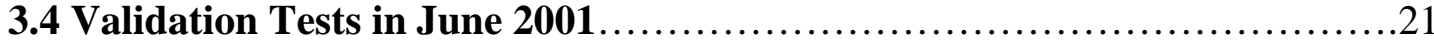

Static Positioning in Differential Mode....................................22

Static Positioning in Undifferential Mode .................................24

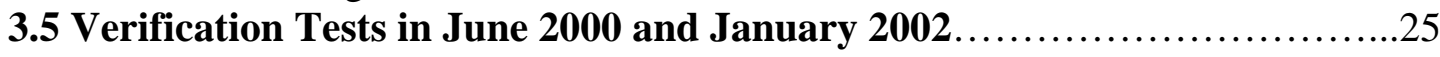

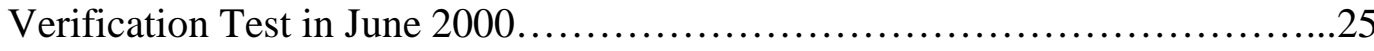

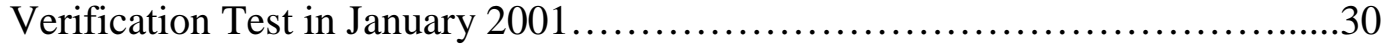

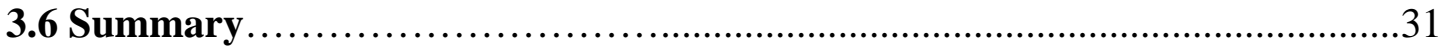


Chapter 4 Development of a Computer Program for Data Collection and Data

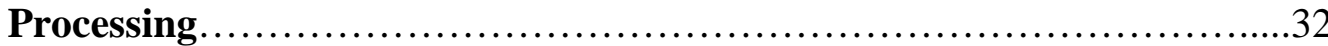

4.1 Overview of the GPS-Trek Computer Program........................... 32

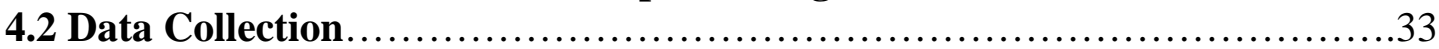

4.3 Data Conversion........................................................... 37

Conversion of UTC Time to Local Time....................................37

Calculation of Travel Time.................................................... 38

Calculation of Travel Distance............................................ 38

Conversion of Longitude and Latitude to UTM Coordinate..................... 38

Calculation of Travel Speed.............................................41

File Conversion.......................................................41

4.4 Data Processing and Data management................................ 42

Map Component......................................................43

GPS Data Display.......................................................43

Insertion of Intersection Marks...........................................44

Selection and Exporting of the GPS Data..................................44

Chapter 5 Sample Size Requirements and Field Procedure for GPS Traffic Data

Collection ........................................................... 46

5.1 Sample Size Requirements..........................................46

Current Methods for Estimating Minimum Sample Size......................46

Estimates of Dispersion................................................50

Modified Equation for Determining Minimum Sample Size...................53

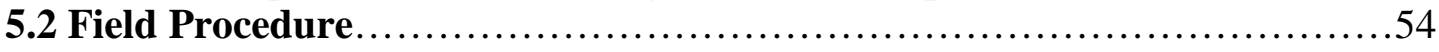

Installation of GPS Data Collection System...............................54

Field Data Collection..................................................... 58

Chapter 6 Use of GPS-Trek in Transportation Engineering Studies................60

6.1 Format of Fundamental Data File and Creation of Data Worksheet.........60

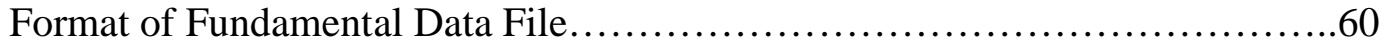

Creation of Data Worksheets...............................................61

6.2 Selection of Study Routes...............................................64

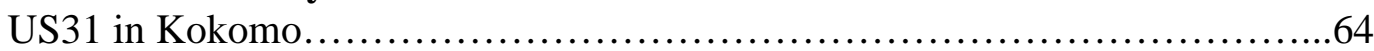

SR26 in Downtown Lafayette.........................................65

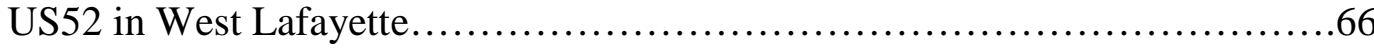

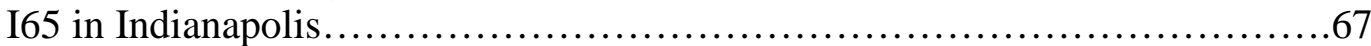

6.3 Application to Travel Time Studies....................................67

Measurements of Travel Time and Distance.................................67

Case Studies..............................................................68

6.4 Application to Travel Speed Studies.................................. 71

Measurement of Travel Speed............................................ 71

Case Studies.............................................................73

6.5 Application to Travel Delay and Congestion Studies....................... 77

Measurement of Travel Delay...........................................77

Measurement of Congestion.............................................. 81

Assessing Congestion at Management Level................................83 
Chapter 7 Conclusions and Implementation Recommendations...................86

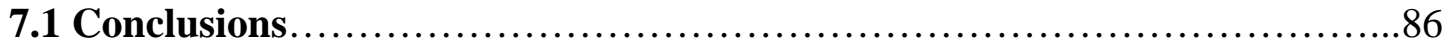

7.2 Implementation Recommendations ................................... 89

References.................................................................. 91 


\section{LIST OF FIGURES}

Figure

Page

3.1 GPS Data without Fully Employed SA................................. 13

3.2 Time Histories without Fully Employed SA............................... 13

3.3 GPS Data with Fully Employed SA............................................

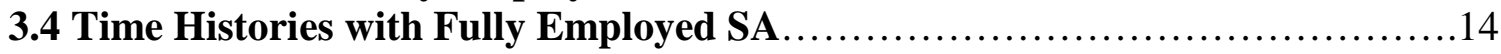

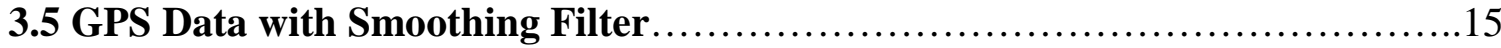

3.6 Time Histories with Smoothing Filter......................................

3.7 GPS Data with OmniSTAR Service Being Enabled......................... 17

3.8 Time Histories with OmniSTAR Service Being Enabled ...................... 17

3.9 GPS Data with OmniSTAR Service Enabled................................ 18

3.10 Time Histories with OmniSTAR Service Enabled .......................... 18

3.11 GPS Data with SA off and OmniSTAR Service Being Enabled.................19

3.12 Time Histories with SA off and OmniSTAR Service Being Enabled............20

3.13 GPS Data with SA off and OmniSTAR Service Disabled...................21

3.14 Time Histories with SA off and OmniSTAR Service Disabled................21

3.15 GPS Data at Q094 in Differencing Mode..................................23

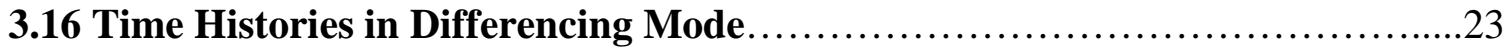

3.17 GPS Data at Q094 in Undifferencing Mode................................25

3.18 Time Histories in Undifferencing Mode................................25

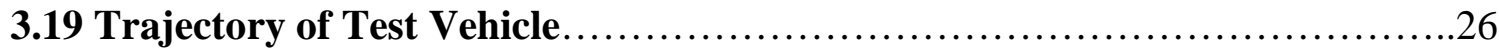

3.20 Trajectory of Test Vehicle on Ohio Street (Eastbound) ......................27

3.21 Trajectory of Test Vehicle on Ohio Street (Westbound) ......................28

3.22 Trajectory of Test Vehicle on Illinois Street (Northbound) .....................29

3.23 Four Corners at the Ohio/Illinois Intersection.............................. 31

5.1 Variations of $t$-Value and $Z$-value with Sample Size.......................50

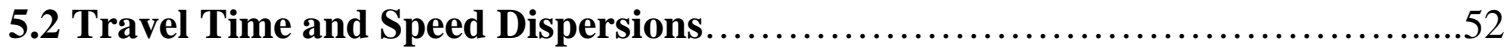

5.3 Minimum Sample Size Computed Using Various Method ....................53

5.4 Minimum Sample Size Computed Using t-Value and Z-Value ................54

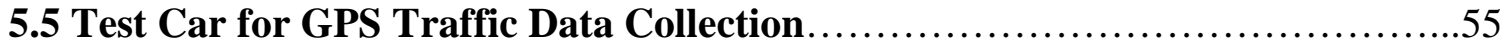

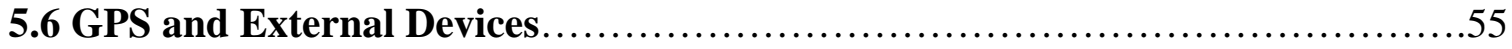

5.7 Installation of GPS and External Devices................................... 57

6.1 Graphical Illustration of Test Segment on US31 in Kokomo...................65

6.2 Graphical Illustration of Test Segment on SR26 in Lafayette.................66

6.3 Graphical Illustration of Test Segment on SR26 in Lafayette.................66

6.4 Graphical Illustration of Test Segment on I65 in Indianapolis................67

6.5 Trajectories Measured Using GPS-Trek on US31 in Kokomo...................69

6.6 Trajectory Measured on SR26 in Lafayette.............................. 70

6.7 Trajectory Measured on US52 in West Lafayette.......................... 71

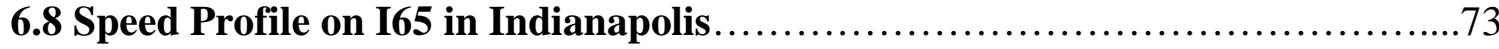

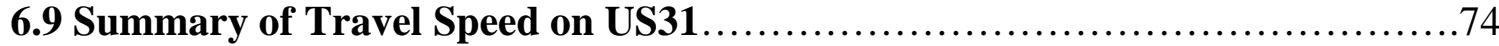

6.10 Summary of Travel Speed on SR26.................................... 75 


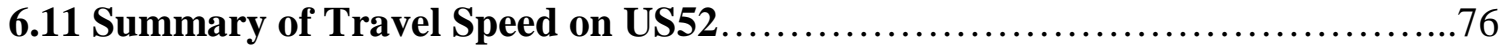

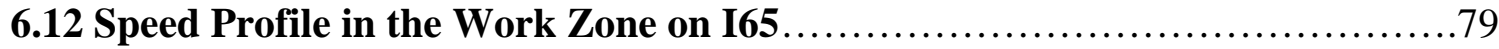

6.13 Speed Profile on US31 ................................................. 80

6.14 Illustrations of Travel and Delay Rates on US31 in Kokomo.................83

6.15 Speed Diagram in a Street System.................................. 85 


\section{LIST OF TABLES}

Table

Page

3.1 Validation Test w.r.t. Q094 with OMNISTAR Differencing Enabled............22

3.2 Validation Test w.r.t. Q094 with OmniSTAR Differencing Disabled............24

3.3 UTM Coordinates of the Four Corners at the Intersection of Ohio with Illinois

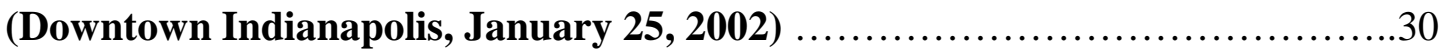

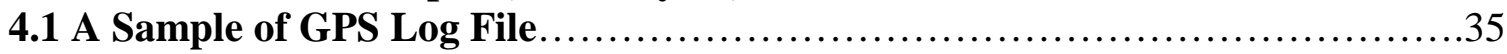

4.2 A Sample of the.$g p s$ File.............................................42

5.1 Minimum Sample-Size Requirements for Travel-Time and Delay Studies......47

5.2 Values of $\varepsilon_{N}$ and $Z_{\alpha / 2}$ for Determining Sample Size..........................54

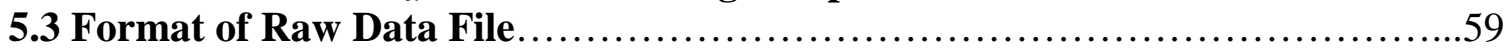

6.1 Fundamental Data Worksheet.............................................62

6.2 Example of A New Column with Additional Special Data...................63

6.3 Travel Rate and Delay Rate for US31 in Kokomo ..........................82 


\section{Chapter 1}

\section{INTRODUCTION}

There are about 11,000 miles highways and hundreds of traffic signal systems that are currently maintained by the Indiana Department of Transportation (INDOT). Due to the rapid growth of economic activities, traffic volumes increase over the years and the performance of the signal systems at some intersections is worsening. In addition, pavements and bridges are deteriorating and many maintenance and rehabilitation projects are underway. As more work zones have been established, the public seem to become more sensitive to the congestion and safety within the work zones. Consequently, it becomes more and more challenging for transportation engineers to maintain the level of service of signalized intersections, reduce possible travel delay, mitigate traffic congestion during peak hours, and enhance safety. In an attempt to provide an efficient and safe highway system, INDOT has made great effort to utilize new technologies to address those potential problems. As part of the Agencies Strategic Plan, INDOT intends to re-time most of existing traffic signal systems and coordinate signal systems over main road segments with heavy traffic in order to allow vehicles, especially vehicle platoons to clear all intersections without significant delay. Also, many measures have been made to address the congestion and safety issues arising due to work zones. Therefore, INDOT traffic engineers have to evaluate signal system performance, prioritize alternatives of improvement, and conduct before and after studies frequently. To accomplish these challenging jobs, it requires a large amount of basic traffic data, such as travel time, travel speed and travel delay.

\subsection{Problem Statement and Research Objective}

Currently, the collection of basic traffic data is mainly conducted by driving on a selected highway section and recording both the total travel time and the time with a speed of lower than the critical speed, such as $5 \mathrm{mph}(8 \mathrm{~km} / \mathrm{h})$. These practices provide a 
direct estimation of travel time and delay, but some problems, such as safety, cost, efficiency and labor, may arise in the course of field data collection. For manual data collection, it usually requires two operators, a driver and a recorder. The manual data collection also requires much time for post process and the whole procedure is time consuming and labor intensive. The automatic data collection currently available uses a laptop computer connected to a distance measuring instrument (DMI) and can record travel distance and time automatically. While it has been thought that one operator can perform automatic data collection, in fact, the field operation is safer with two operators, especially under severe weather and traffic conditions. In addition, the data files may not be reusable because of the limits of the software.

With the emergence of Global Positioning System (GPS) technologies, it appears that traffic data collection can be performed more efficiently and safely. Combining geographic information system (GIS) or other map displaying technologies, GPS provides a promising tool for data collection and for addressing certain traffic issues, such as traffic impact, delay measurement and congestion management. Although effort has already been made to investigate use of GPS technologies in transportation studies by New York, Louisiana and North Carolina, more research work is required to address the application issues due to the following reasons. First, GPS technologies have advanced greatly in the past two years and the capabilities of the GPS hardware and software have been enhanced tremendously. It is claimed that the latest GPS receivers, such as $\operatorname{AgGPS}^{\mathrm{TM}}$ 132, provide sub-meter Differential GPS (DGPS) position accuracy and allow us to utilize GPS technologies much more efficiently. Secondly, traffic delay and congestion are usually addressed at two levels: management and project levels. While those two users share the same fundamental data, they require different resolution, accuracy and information output. It is a great challenge for us to explore an efficient algorithm to collect, process, store and manage data that may satisfy the specific needs at these two different levels. 
The objective of this study is to investigate use of the GPS technologies to improve efficiency of INDOT traffic data collection, enhance field operation safety and

reduce potential human errors. Primary emphasis is given to use of Trimble $\operatorname{AgGPS}^{\mathrm{TM}} 132$ receiver to acquire the above-mentioned basic traffic data so as to generate a reusable data file for transportation studies. This type of data file is essential for providing INDOT traffic engineers with consistent information to assess the performance of signal systems and conduct before and after studies. Secondary emphasis is given to use of the created data files in specific transportation studies, such as travel time and delay studies, work zone studies and congestion studies. It should be pointed out that INDOT has already created base map for state highway system. While this base map may not satisfy the resolution requirement for certain traffic studies, it is difficult to create a new base map because of the cost in terms of money, time and manpower. Therefore, it is logical for us to investigate a procedure to create general and reusable data files that can be utilized for various transportation studies on the basis of the existing highway base map.

\subsection{Research Scope and Approach}

This study focused on four areas. The first area was to examine GPS techniques, including selection and testing of GPS equipment and DGPS data service. The second area was to develop a computer program for field GPS data collection, processing and management. The third area was to conduct extensive field data collection so as to test the program, identify data variations, evaluate sample size requirements and establish a field operation procedure. The final area was to investigate use of the procedures and programs developed in this study for transportation studies. In order to accomplish the research objective, the following research approach was employed in the course of performing this study.

First, an extensive review of literature was performed to examine the progress of GPS techniques and its application to field data collection. Emphasis was given to GPS equipment and service, procedures for GPS traffic data collection and use of those data in transportation studies, such as travel delay studies and congestion management. 
Secondly, a GPS receiver with differential correction function, $\operatorname{AgGPS}^{\mathrm{TM}} 132$, was purchased, the OmniStar DGPS data service was ordered, and the external devices, such as an IBM Thinkpad laptop computer, power inverter and power supply, were secured. All devices were tested and sources of errors were verified. Meanwhile, a procedure was derived and computerized for GPS data collection and processing. The computer program was named GPS-Trek, consisting of two components that were developed for data collection, data storing, file conversion, data processing, data management and mapping. Following the aforementioned work, a working system was established for traffic data collection. This system, consisting mainly of the $\operatorname{AgGPS}^{\mathrm{TM}} 132$ devices and an IBM Thinkpad laptop computer installed with the GPS-Trek, was tested at selected test sites that included highway corridors with uncoordinated or coordinated signal systems and freeway work zones. Next, extensive data collection was conducted. During field data collection, the initial GPS-Trek was modified and the format of data file was optimized. Data variations were also examined in order to establish sample size requirements for field data collection. Finally, this study investigated application of the data files and worksheets created using the GPS-Trek to various transportation studies and discussed use of those data for evaluating travel delay and congestion.

\subsection{Proposed Hardware and Software for the System}

\section{GPS Devices}

- $\mathrm{AgGPS}^{\mathrm{TM}} 132$ receiver: fast rate and DGPS capabilities

- $\mathrm{AgGPS}^{\mathrm{TM}} 132$ antenna

- OmniStar subscription of satellite data service

- Magnetic mount for antenna

- 5-meter ruggedized antenna cable

- Data/power cable

\section{Laptop Computer}

- Process speed: $300 \mathrm{MHz}$ or higher

- Memory: $64 \mathrm{MB}$ or more 
- Hard drive space: $100 \mathrm{MB}$ or more

- Operating system: Windows 95, 98, NT or 2000

- Communication port: one serial port

\section{Software}

The GPS-Trek installation CD

\section{$\underline{\text { Accessories }}$}

- DC-to-AC power inverter: DC $13.8 \mathrm{~V}$ and AC $120 \mathrm{~V}$

- DC power supply: AC $120 \mathrm{~V}$ and DC $13.8 \mathrm{~V}$ 
Chapter 2

\section{SELECTION OF GPS DEVICES FOR TRAFFIC DATA COLLECTION}

GPS position technology provides a fast, flexible, and relatively inexpensive technique to determine one's position and velocity in real time or after the fact. GPS positioning is based loosely on three-dimensional positioning using trilateration related techniques (1). The space segment consists of 24 GPS satellites orbiting the Earth in half sidereal day orbits (about 12 hours). The length of the orbital period guarantees that the GPS satellites will repeat their orbits daily for an Earth bound observer. However, every day the GPS satellite will trace out the same sub-orbital track four minutes earlier. This causes a daily repeatability in the satellite geometry with respect to an Earth bound observer. The quality of the available satellite geometry directly impacts the quality of the positioning obtained. A parameter that expresses the time varying quality of the position determination is the so-called DOP factor, or Dilution of Precision. The DOP factor is the ratio between the standard deviation of one or more positional parameters (longitude, and/or latitude, and/or height). In order to have successful position determination, the satellites (time varying) positions re assumed to be known.

\subsection{GPS Observables}

Two major types of observables are available to GPS users: travel-time measurements through coded signals on a carrier wave, and phase measurements on the carrier wave itself.

\section{Coded Signals on a Carrier Wave}

The first one is based on a coded signal transmitted on a carrier wave. The coded signal consists of a random sequence of zeros and ones (Pseudo Random Noise (PRN) code). Each satellite broadcasts its own code, enabling satellite identification. Cross correlation in time with an identical coded signal generated by a GPS receiver, and the 
evaluation of time tagged information of the signal leaving the satellite and arriving at the receiver, results a measured range between the satellite and the receiver. Since two clocks are involved, keeping time in principally different time systems, the technique is called pseudo-ranging because the clock offsets between the two clocks is unknown. Since the receiver does not relay the signal back to the satellite, pseudo-ranging can be best described as one-way ranging with two independent clocks.

Positioning becomes possible if a receiver receives coded signals from at least four GPS satellites. Four range equations can be formed to solve for four unknown parameters: the three dimensional position of the receiver and the clock offset between the two clocks. Since each satellite carries its own independent clock, the GOPS user through its receiver has access to the time corrections for each individual satellite clock to a common (GPS) time system. That means that each receiver has to solve for only one single clock offset parameter between the receiver clock and the common to all GPS satellites common time system. Since in this trilateration model it needs to be assumed that the satellite positions are known, the receiver receives and decodes as part of the coded message information about the position of each individual GPS satellite.

At the start of this research project the Department of Defense deliberately degraded the satellite position and clock information for strategic purposes: the Selective Availability, or SA. This and the nature of the measured pseudo-ranging led to an overall accuracy of positioning anywhere between 5 and 50 meters (15-150 feet). The (known) code broadcast by the GPS satellites, is known as Coarse Acquisition (C/A) code. This code is modulated only on one of the two frequencies, and is accessible to civilian users. The available Precise ( $\mathrm{P}-$ ) code available generally only to military users has not been considered for this research. Receivers capable of deciphering C/A code range in price between $\$ 50$ and several thousands of dollars.

\section{Phase of the Carrier Wave Signal}

The second observable, generally used for high precise positioning applications, involves the measurement of the phase on one or both carrier waves. Having a 
wavelength of the carrier waves in the order of two decimeters, and phase easily being measured with $1 \%$ or better, this measurement leads to a range type of observation with an accuracy of several millimeters. These types of receivers that exploit the phase observable(s) to the fullest extent cost generally from a few thousand, to tens of thousands of dollars. Therefore this observable has not been considered in this research project.

\subsection{Differential GPS or Single Point Positioning}

Knowledge of the satellite positions is mandatory to be able to resolve one's position from pseudo-range observations. Also, these satellite positions are not errorless, either because of the fact that the predicted positions are computed with partially known forces, or because of the deliberate SA errors. Consequently, one has to resort to special data collection and analysis techniques to bring the crude PR positions $(5-50 \mathrm{~m})$ to acceptable accuracy levels for the user (2-5m).

\section{Differencing Techniques}

The geometry of GPS trilateration is such that GPS receivers are relatively close to each other (few kilometers), whereas the satellites are far removed from the receiver network. Granted that this is a very unfavorable geometry, the advantage is that the effect of (deliberate) satellite errors almost equally effect GPS receivers in the same neighborhood.

Differencing pseudo-ranges collected at the same time by different receivers, the first order effect of systematic errors largely cancel. This leads to two items for serious consideration: the timeliness of the GPS data collection and analysis, and the organization of GPS differencing operations. The differentially analyzed GPS

observations, especially in real time operations are known under the acronym DGPS (Differential GPS). 
Regarding the timeliness of the GPS data one may differentiate between two issues:

1. Perform the differencing after the fact in a batch processing. This can be done semi automatically, or automatically, However, this option involves the storage (in the field), relaying, analyzing, and archiving (in the office) of GPS data.

2. Perform the differencing in real time. It may well be that real time availability of GPS positions is not necessary, the large advantage of this option is that organizational problems concerning the storage, relay, and archiving of GPS data is circumvented.

Two major solutions regarding the GPS differencing options have been looked at in this project:

a. The organization, i.e. INDOT, is itself responsible for collecting data from other GPS receiver than the roving receiver in the field. The organization itself sets up an in-house differencing facility. Many years ago, the Texas Department of Transportation has opted for such an approach. Since the State of Indiana would have to build up such a venture from the ground up, it was decided against such solution.

b. INDOT buys correction information from an organization that monitors the time varying systematic errors through a network of GPS receivers, the socalled the Wide Area Differential GPS network (WADGPS). Generally these correction data are available through radio links, FM radio stations, geostationary satellites, or recently through cell phones. Since INDOT did not need to build a new infrastructure for such a facility, this solution was considered to be the optimal one.

\section{Single Point Positioning}

With the research well underway, and decisions made for investments in the purchase of GPS equipment and the subscription to a DGPS service, a new possibility opened up on May 1, 2000: then President Clinton removed SA from the coded signals 
for an indefinite period of time. This lead to a series of additional tests reported upon in Chapter 3.

\subsection{Selection of GPS Receivers and DGPS Service.}

Initially a wide variety of GPS receiver manufacturers have been looked at:

- Ashtech/Magellan,

- Garmin,

- Javad/Topcon,

- Marconi,

- Novatel,

- Rockwell Collins, and

- Trimble.

Also a series of DGPS services have been looked at:

- ACCQPOINT Communications Corporation by CUE,

- Continuously Operating Reference Stations (CORS) by the National Geodetic Survey (NGS),

- OmniSTAR by John E. Chance \& Associates (JECA),

- VISION GPS/GIS by Rockwell Collins, FLYING FLAGMAN by Del Norte,

- $\quad$ NovAtel (AG-20), SatLoc (TerraStar),

- $\quad$ STARLINK, DCI Services, TDSWAY, TDS SOLO, etc.

During the selection process in the first half year of the two-year research period it became clear that the selection of receiver and the selection of the DGPS service should not probably be separated. User friendly implementation tasks during the start of a DGPS service while having specific DGPS receivers at hand became more important parameters than cost, etc. Another issue that became a deciding issue was the data rate with which GPS data could be collected and differenced. Despite the fact that the GPS data collected by NGS' CORS stations is available free of charge to the user, the coverage of CORS stations in the State of Indiana is poor. Moreover, the CORS data rate available for users 
were at best at the 5 seconds interval, but more likely at 30 seconds interval. This would be insufficient to difference GPS field data collected in vehicles traveling at speeds up to $65 \mathrm{mph}$.

It was decided to select the $\operatorname{AgGPS}^{\mathrm{TM}} 132$ receiver (2) from Trimble, coupled with the OmniSTAR service. The cost of the receiver, including $10 \mathrm{~Hz}$ data rate capability, and external antenna with 5 meter cable, was about \$5000, whereas OmniSTAR's DGPS service ran at \$800/year. Investment into a Laptop PC (\$2000) for data collection would complete the system. The project used an existing INDOT laptop during its experiments.

The AgGPS ${ }^{\mathrm{TM}} 132$ receiver is a 12 -channel C/A code receiver. The update rate is $1 \mathrm{~Hz}$, with $10 \mathrm{~Hz}$ capability. Expected differential position RMS accuracy is 1 meter or better horizontally while tracking at least 5 satellites with a PDOP better than 4 . The expected speed accuracy is about $0.1 \mathrm{mph}(0.16 \mathrm{kph})$. 
Chapter 3

\section{AgGPS $^{\mathrm{TM}} 132$ VALIDATION TESTS}

The Trimble AgGPS ${ }^{\mathrm{TM}} 132$ receiver has been submitted to a series of validation tests. The unit arrived at Purdue University in March 2000. That means that some tests have been performed before the Selective Availability (SA) was turned off at midnight on May 1/2, 2000. As a matter of fact, the OmniSTAR Differential Service was started in the morning of May 1, at exactly the same time President Clinton gave a press conference announcing that SA would be turned off at that very midnight. In section 3.1 the tests are described before May 1, 2000 with Selective Availability turned on, and in sections 3.2 and further, the tests after May 1, 2000 with Selective Availability turned off.

\subsection{Validation Tests before May 1, 2000 (SA on, OmniSTAR off)}

\section{First Data: April 2000}

The very first data with the newly acquired $\operatorname{AgGPS}^{\mathrm{TM}} 132$ was collected on Saturday, April 15, through Sunday, April 16, 2000. The GPS data, gathered during one hour in stationary mode, was directly streamed to a hard disk using the HyperTerminal software. The data seemed to be remarkably free of the effects of Selective Availability (SA), although these deliberately induced errors were still present: the longitude (east) component does not seem to wander more than 2 meters from the "true value". The latitude shows a more normal 'SA-behavior": $-11 \mathrm{~m}<$ dLatitude $<6 \mathrm{~m}$. Figure 3.1 illustrates the large excursions, particularly in latitude.

Figure 3.2 shows the time histories in longitude, latitude, and recovered velocity. Along the abscissa axis it is shown that the dataset was collected around midnight (Saturday April 15 to Sunday April 16), between 23:20:00 EST and 00:20:00 EST (local time). From the relatively small variation in latitude (about 17 meters) it can be deduced that SA was not fully employed at the time of the experiment. The velocity plot (the 
speed-receiver time plot at the bottom in Figure 3.2), confirms that the (static) receiver correctly determines that it is not in motion.

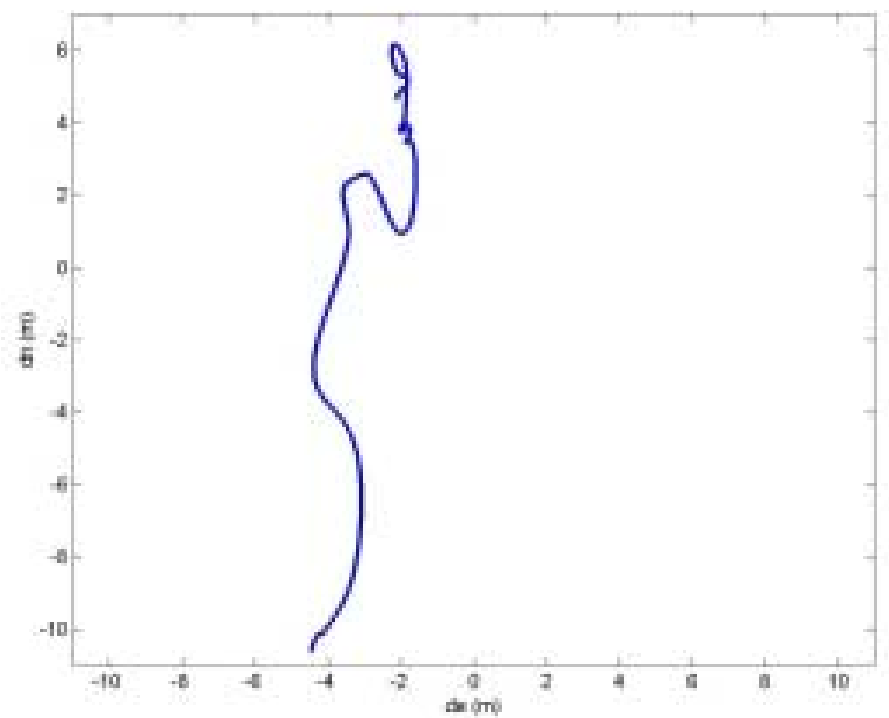

Figure 3.1 GPS Data without Fully Employed SA
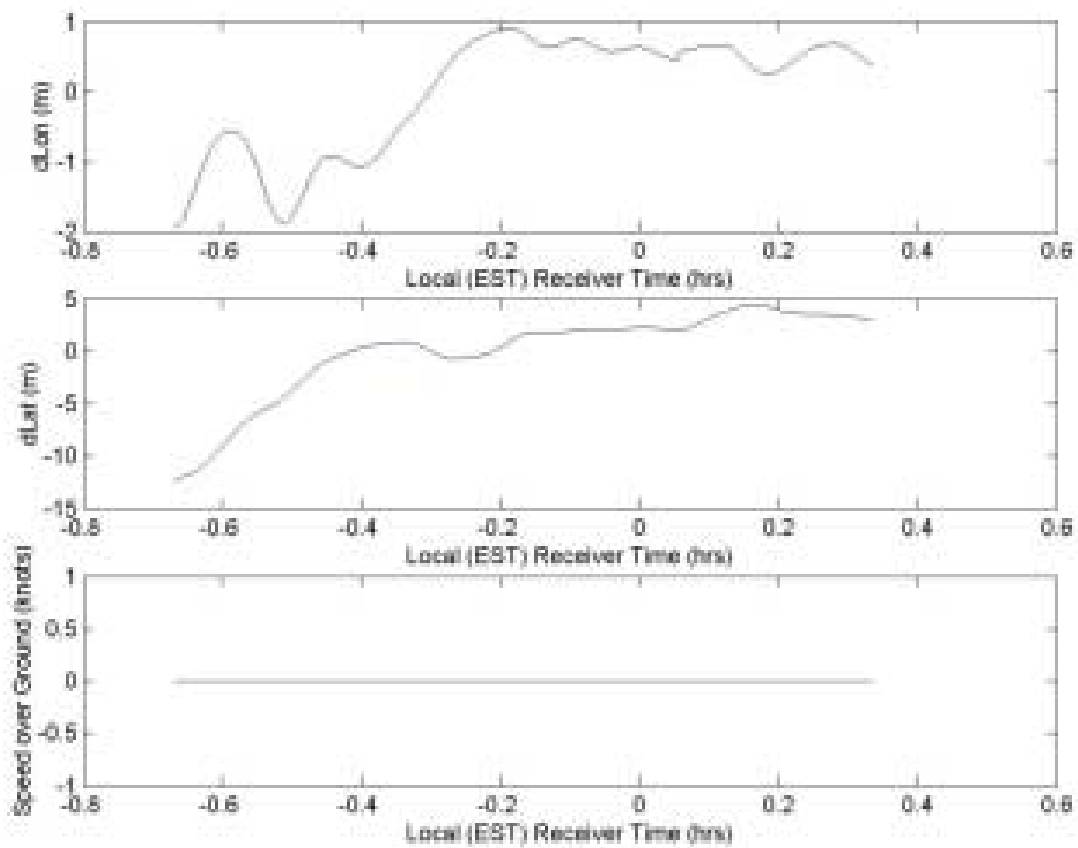

Figure 3.2 Time Histories without Fully Employed SA 


\section{Selective Availability: May 1,2000}

Two weeks later, a second test was performed on Monday, May 01, 2000, between 09:42:50 EST and 09:53:10 EST. Unknowingly, this test happened to be on the last day that Selective Availability was switched on. During this test one could clearly see "SA-at-Work". In a mere 10 minutes the position wanders off by more than 200 meters, see Figure 3.3. The time history plots confirm the sometimes wild position swings. These wild and rapid variations show as "phantom" velocities in the time history velocity graph, see Figure 3.4. The plotted velocities are phantom velocities since the $\operatorname{AgGPS}^{\mathrm{TM}} 132$ receiver was NOT in motion but stationary. The data rate was $1 \mathrm{~Hz}$.

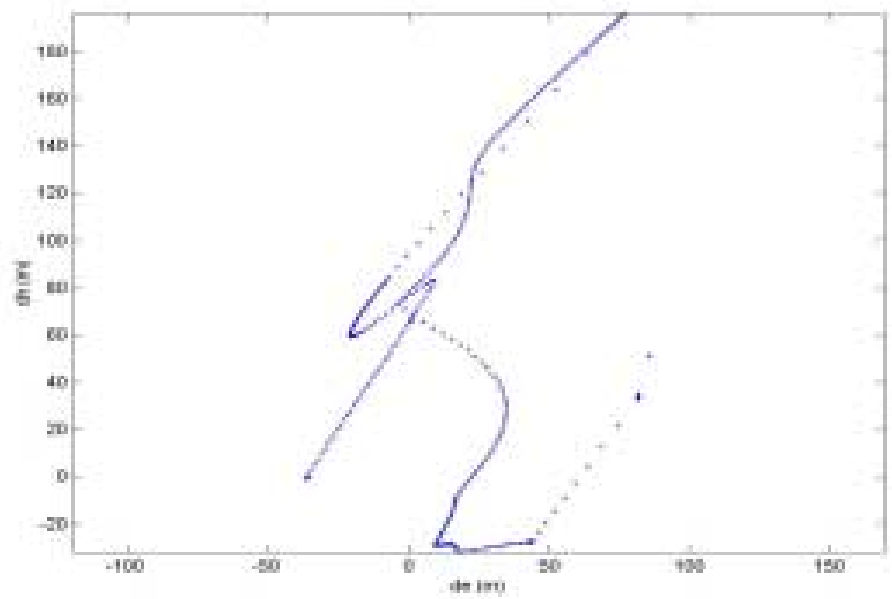

Figure 3.3 GPS Data with Fully Employed SA
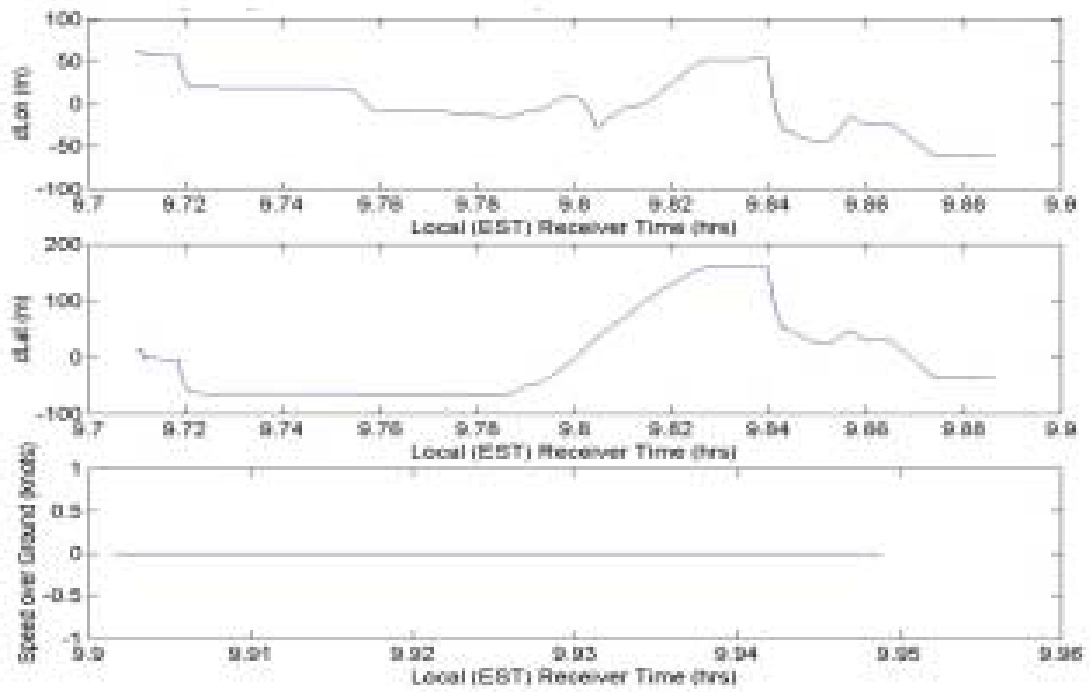

Figure 3.4 Time Histories with Fully Employed SA 


\section{Smoothing Filter: May 1, 2000}

On Monday, May 01, 2000, the effect of the built-in smoothing filter was investigated. The test was taken minutes after the SA test, between 09:54:00 EST and 09:59:10 EST, see previous paragraph. Unknowingly, this test happened to be on the last day before Selective Availability was to be switched off. During this test one could clearly see "SA-at-work". In a mere 10 minutes the position wanders off by about 100 meters, see Figure 3.5.

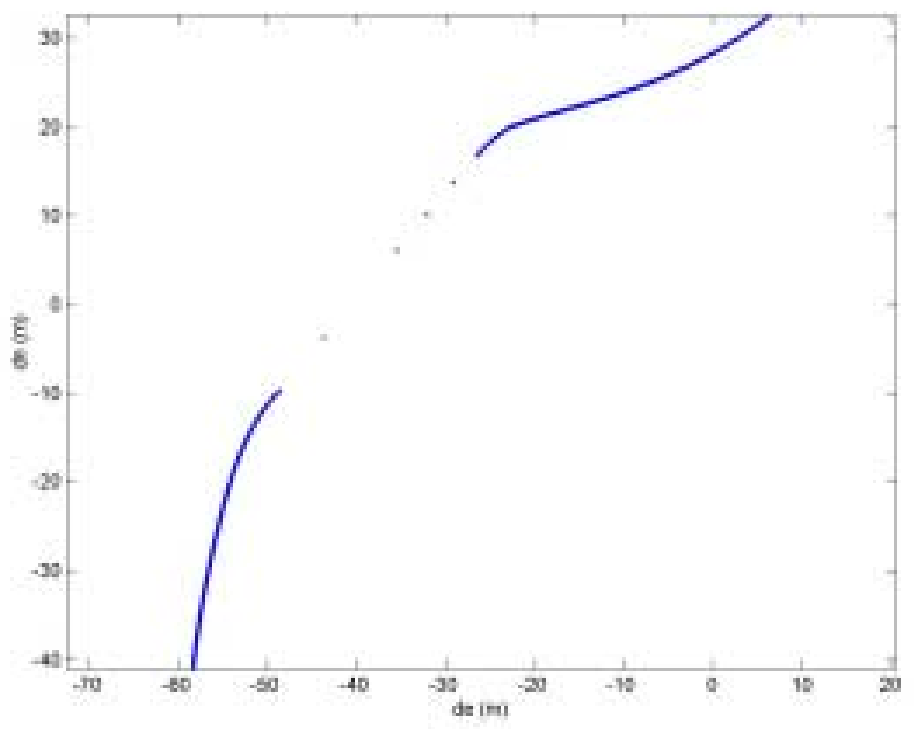

Figure 3.5 GPS Data with Smoothing Filter

The time history plots confirm that the smoothing filter "looses its grip" of the (static) position of the $\mathrm{AgGPS}^{\mathrm{TM}} 132$ GPS receiver: the filter allows small and smooth variations of the continuously updated position (remember the GPS receiver is stationary during this test). However, the discrepancy between the filter allowable position and the actual determined position becomes so large that the filter has to give up the small stepwise updates of position, and a large change, or jump in the position occurs. These phenomena show up as a large discontinuity in the time history plots. During this test the sudden shift occurs at 9.9425 =09:56:33 EST (local time), see Figure 3.6. The data rate was $10 \mathrm{~Hz}$. 

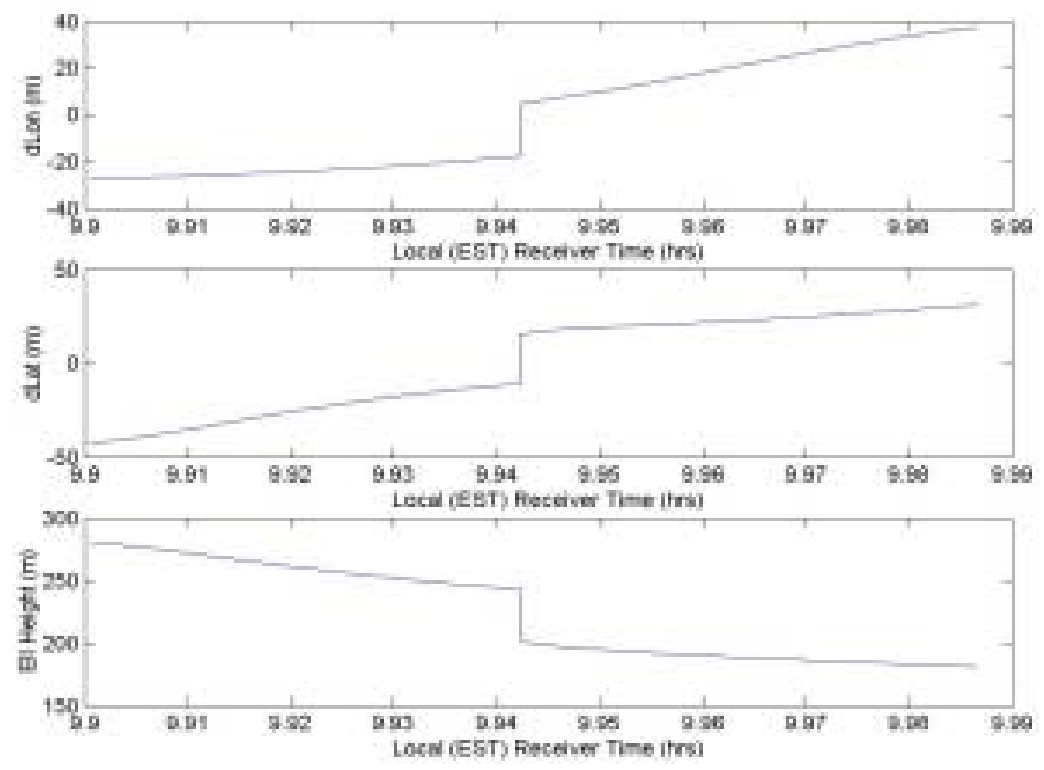

Figure 3.6 Time Histories with Smoothing Filter

\subsection{Validation Tests on May 1, 2000 (SA on, OmniSTAR on)}

\section{Data while OmniSTAR Service Being Enabled: May 1, 2000}

Figures 3.7 and 3.8 show plots of a 40 minute data set, collected in the morning of May 1, 2000, between 10:08:15 EST and 10:48:00 EST. During this time one of the authors of this report (van Gelder) was in contact by phone with a representative of the OmniSTAR organization. By phone call a code is transferred that is needed to be brought into the $\mathrm{AgGPS}^{\mathrm{TM}} 132$ GPS receiver for activation of the OmniSTAR differential service (3). Before about $10.55 \mathrm{hr}=10: 33$ EST the position of the GPS receiver was plus or minus 30 meters off its reference position. The reference position is represented by the origin $\{0,0\}$ in Figure 3.7, and the zero reference time line dLon $=\mathrm{dLat}=0$ meters in the top and middle plots in Figure 3.8. The data rate was $1 \mathrm{~Hz}$.

Figures 3.7 and 3.8 show clearly that the OmniSTAR had been activated at 15:33 UTC ( $=10: 33$ EST). In Figure 3.7 this is apparent by the jump of the trajectory towards the center of the graph, which is the origin $\{0,0\}$ or the reference position of the receiver. In the top and middle plots in Figure 3.8, the time line jumps the zero reference line. 
The irony is, that while we were in telephone contact with personnel to activate the OmniSTAR service (Figures 3.7 and 3.8), President Clinton held simultaneously a press conference announcing that that very night SA would be switched off (4).

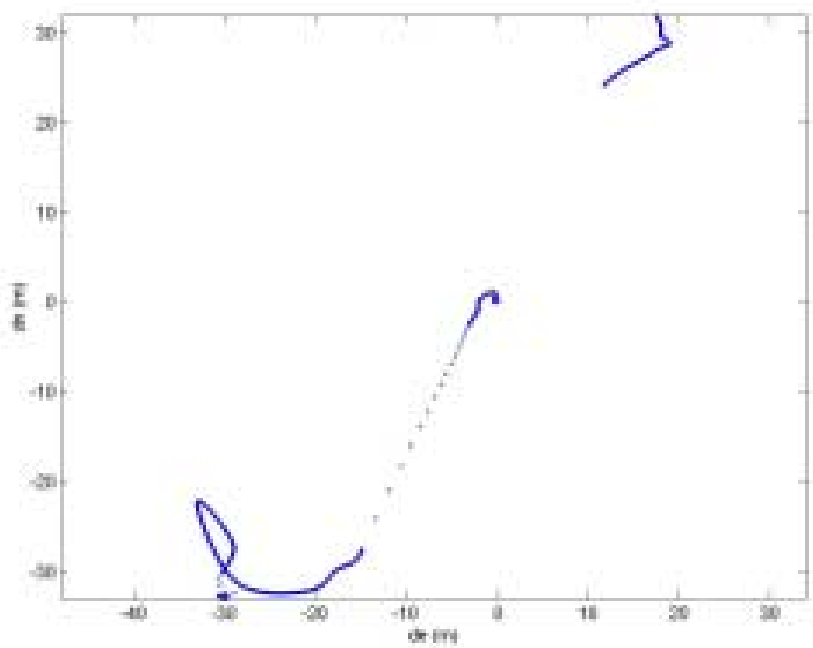

Figure 3.7 GPS Data with OmniSTAR Service Being Enabled
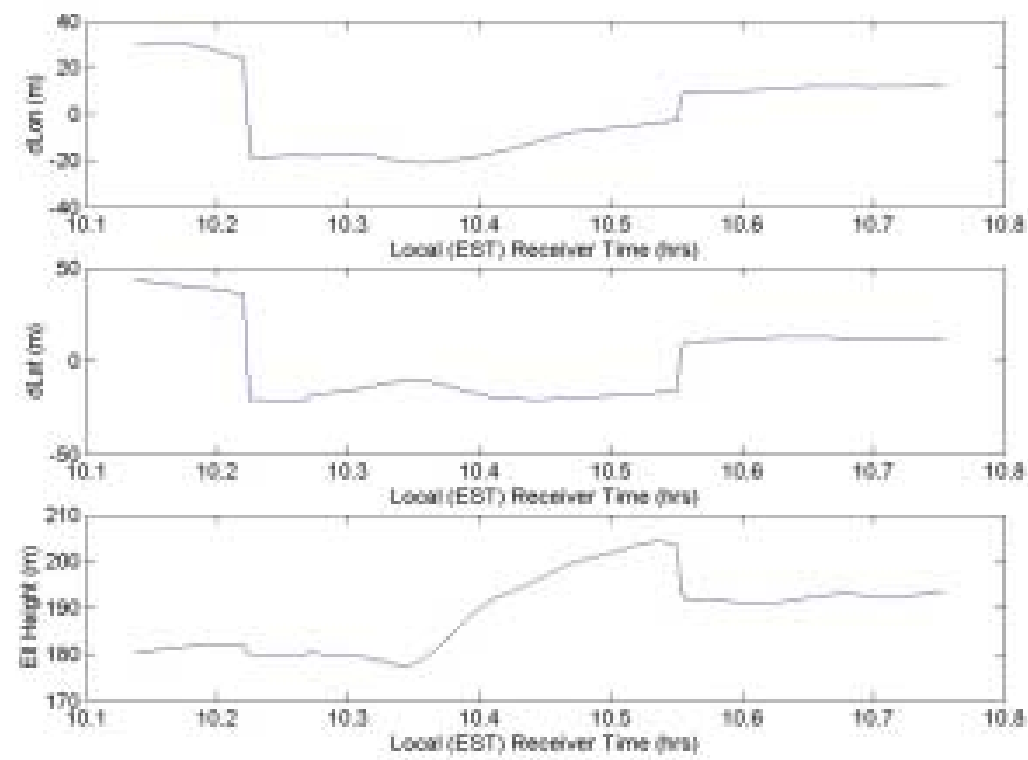

Figure 3.8 Time Histories with OmniSTAR Service Being Enabled

\section{Data after Enabling OMNISTAR Service: May 1, 2000}

Figures 3.9 and 3.10 show the dramatic improvement, created by OmniSTAR's capability to greatly reduce the effects of SA. The 15 minute dataset, collected between 
11:14:50 EST and 11:30:10 EST, came within one hour after the OmniSTAR differential had been activated. It was the morning of May 1, 2000, while SA was still activated by the Department of Defense. The deviations from the reference position are less than 1.5 meters ( 5 feet). An hour before, right before the differential service was activated, these deviations were still in the 30 meter range, see Figures 3.7 and 3.8.

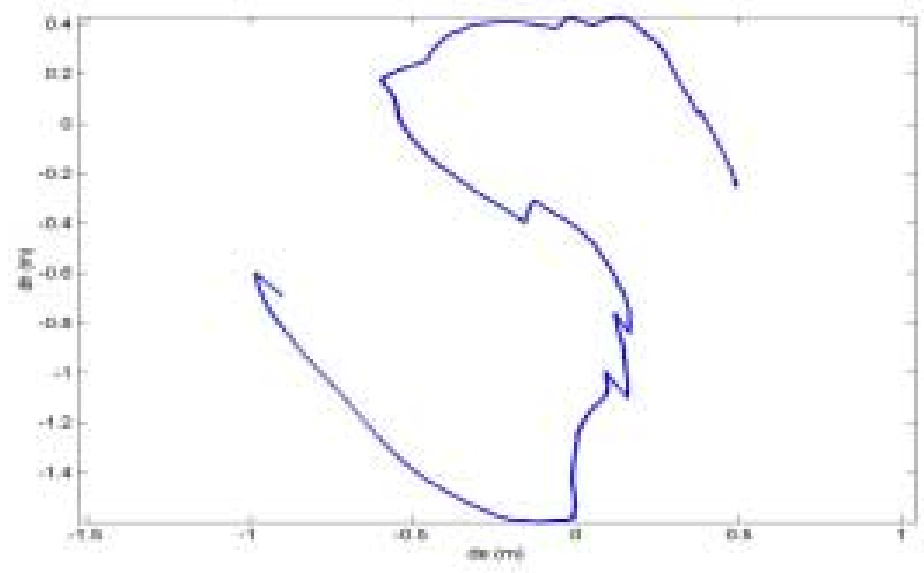

Figure 3.9 GPS Data with OmniSTAR Service Enabled
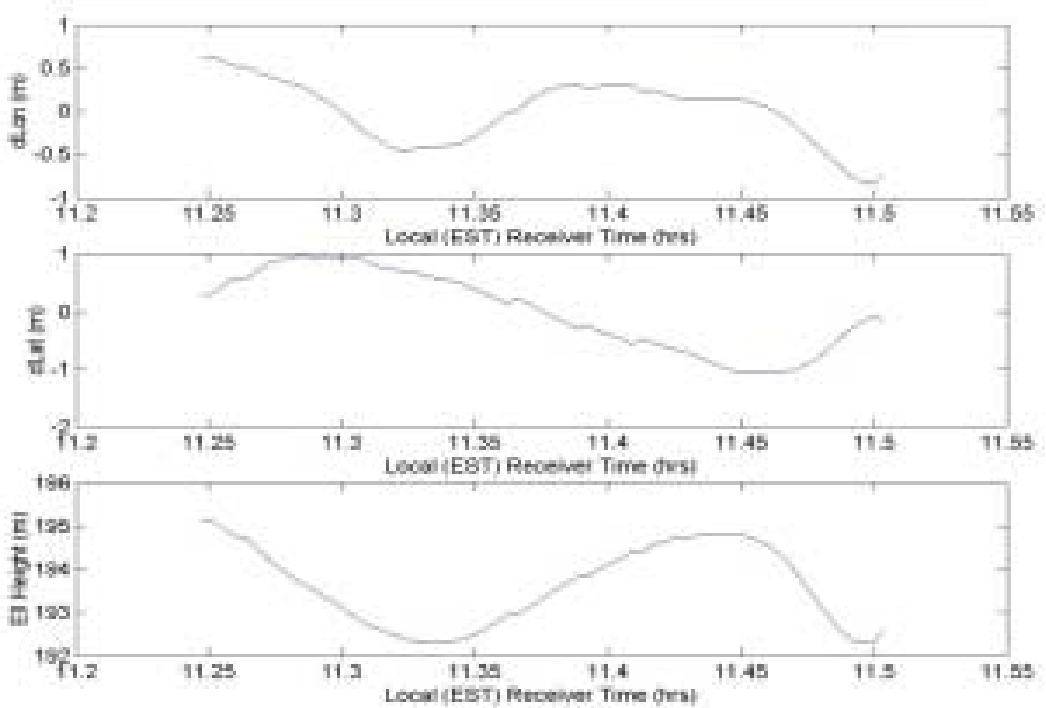

Figure 3.10 Time Histories with OmniSTAR Service Enabled

The somewhat jagged trajectory in Figure 3.9 or Figure 3.10, is caused by the fact that different subsets of the 24 GPS satellite constellation were used in real time. The 
small discontinuities are caused by shielding of the satellites, or by the fact that a subset of the GPS satellite constellation sets below, or rises above the horizon. The data rate was $1 \mathrm{~Hz}$.

\subsection{Validation Tests on May 2, 2000 (SA off, OMNISTAR on/off)}

\section{SA off, while OmniSTAR Service Being Enabled: May 2, 2000}

A 20 minute dataset was collected on Tuesday May 2, 2000, between 06:39:50 EST and 07:00:10 EST, while the receiver was in a stationary position. Since the dataset is collected after midnight between May 1 and 2, 2000, the selective availability has been switched off by the Department of Defense.

Figures 3.11 and 3.12 show that the deviations with "SA off", and "OmniSTAR on" are even better than the day before, see Fig. 9. The deviations of the position of the receiver are well within one meter from the reference position. It was one and a half meter on the day before (May 01, 2000), when we had "SA on" and "OmniSTAR on". The data rate was $1 \mathrm{~Hz}$.

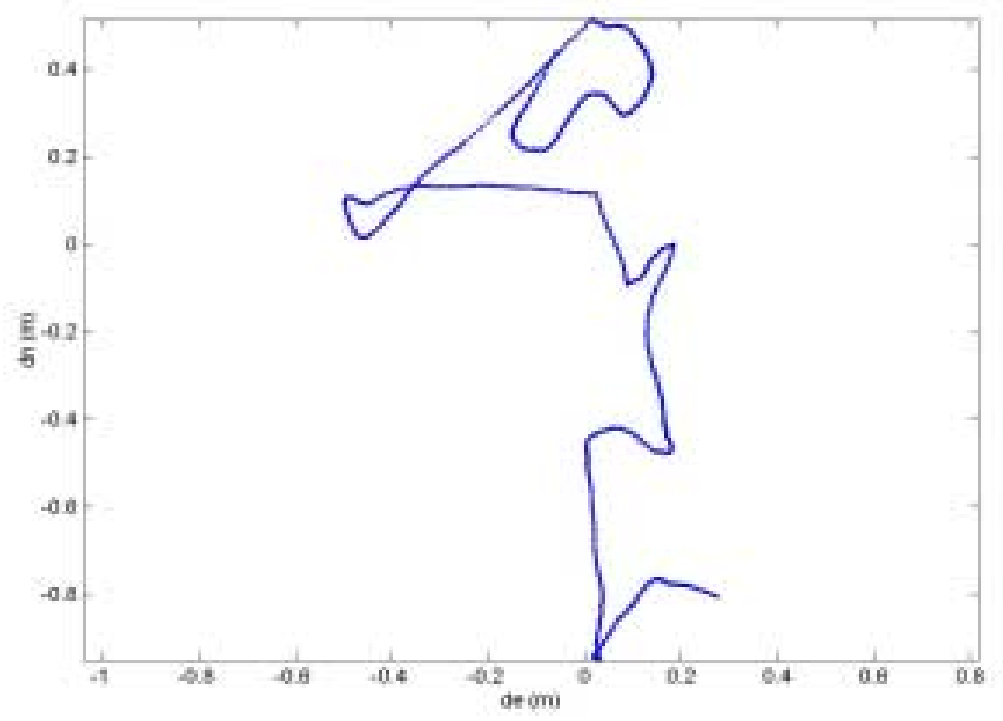

Figure 3.11 GPS Data with SA off and OmniSTAR Service Being Enabled 

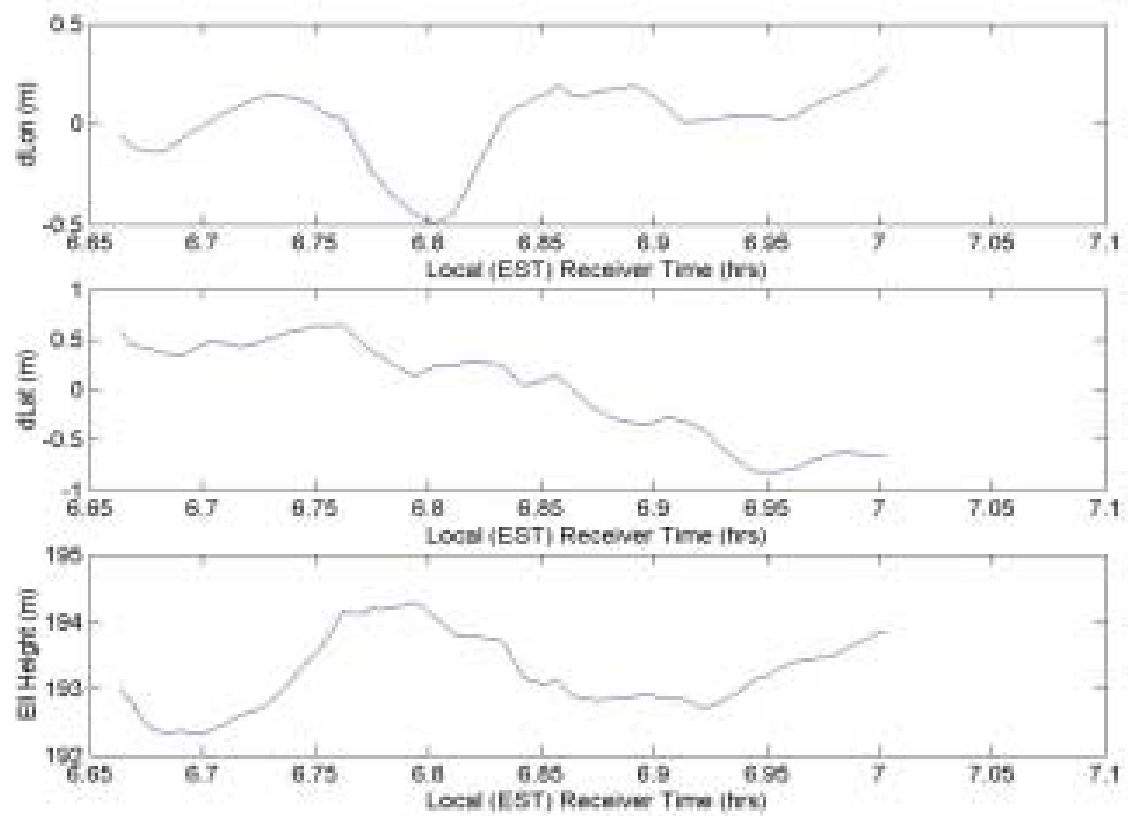

Figure 3.12 Time Histories with SA off and OmniSTAR Service Being Enabled

\section{SA off, while OmniSTAR Service Being Disabled: May 2, 2000}

The GPS AgGPS ${ }^{\mathrm{TM}} 132$ receiver has the capability to collect positions while suppressing any differential service. On May 2, 2000, despite the fact that SA switched off, a 20 minute dataset was collected while the OmniSTAR corrections were being ignored. The dataset was collected between 07:04:50 EST and 07:25:10 EST, minutes after the previous test when the differential collections were applied. The stationary position does not wander more than 1.2 meters from the reference position, see Figures 3.13 and 3.14 .

Figure 3.13 and the top and middle plots in Figure 3.14 show the typical almost linear behavior of the deviation between the GPS recovered position and its reference position. The 20 minute dataset forms, as it were, a snapshot of a trajectory which has a much larger (circular) type of motion. Later this phenomenon would be confirmed again and again, e.g. in Figure 3.17. 


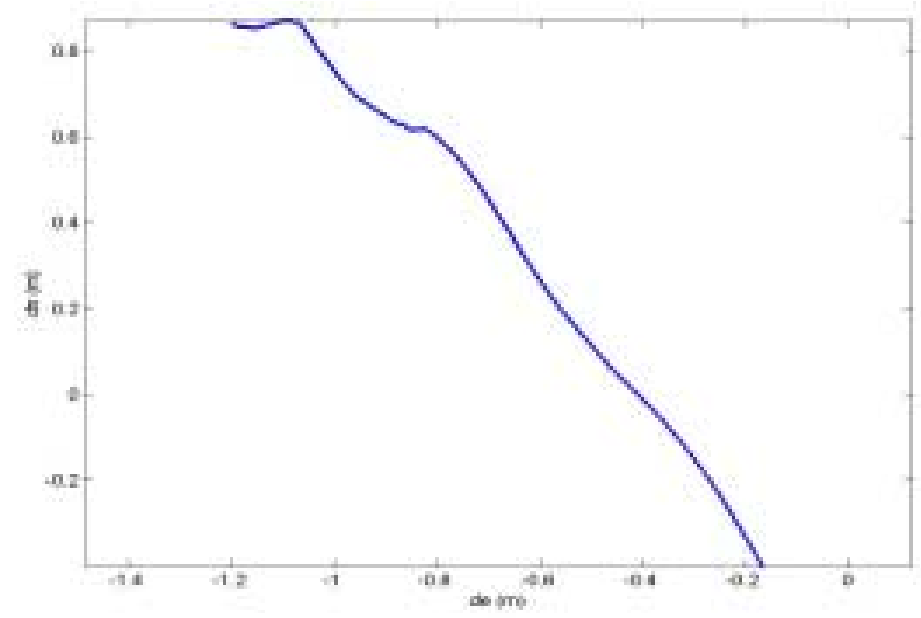

Figure 3.13 GPS Data with SA off and OmniSTAR Service Disabled
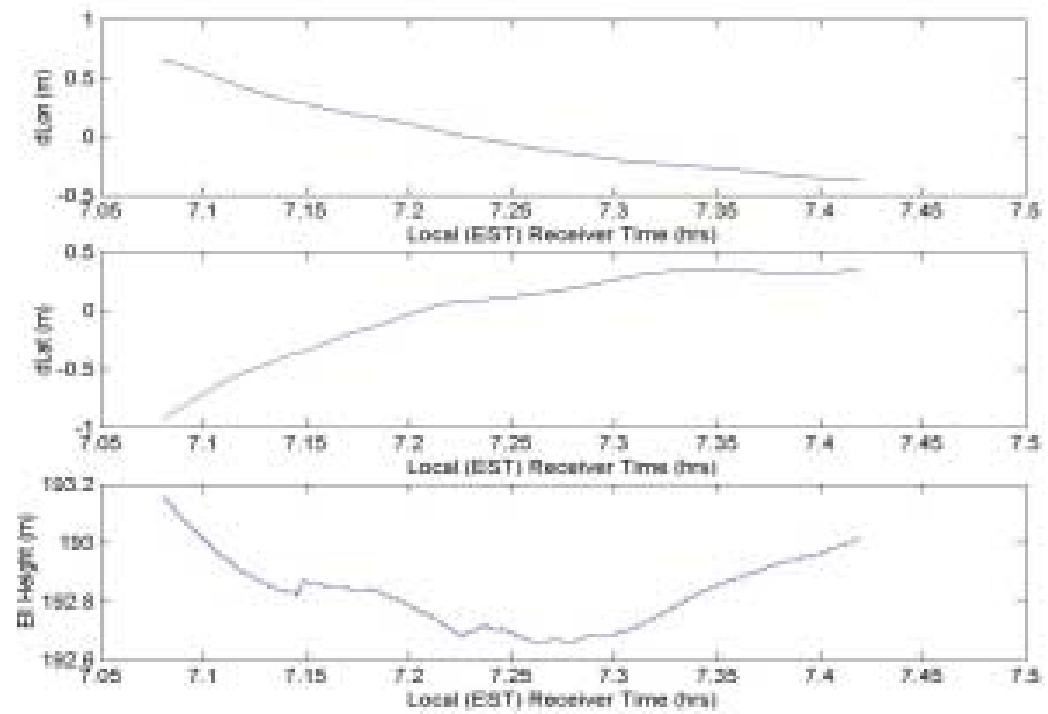

Figure 3.14 Time Histories with SA off and OmniSTAR Service Disabled

\subsection{Validation Tests in June 2001}

Since it is harder to judge the absolute and relative positional accuracy in differenced and undifferenced mode while the receiver is in motion, it was decided to test the $\mathrm{AgGPS}^{\mathrm{TM}} 132$ receiver against a point of the Indiana High Accuracy Reference Network (HARN). The validation tests as reported in the previous sections were with respect to a stationary, but arbitrary position. The test that took place on June 22, 2001, was performed with the same settings as if the receiver was used in motion. For instance, 
the velocity of the (static) receiver was continuously monitored. The HARN point chosen was Q094, in front of the Purdue Airport Terminal.

\section{Static Positioning in Differencing Mode}

The first set of data was collected for about 15 minutes, between 16:01:50 UTC and 16:17:08 UTC (in local time: between 11:01:50 EST and 11:17:08 EST), with the OmniSTAR differencing capability enabled. The data revealed that the precision (internal consistency), measured by the standard deviation around the mean was much higher than the r.m.s., computed with respect to the given coordinates of the HARN station. This was especially true in the north direction, see Table 3.1 .

Table 3.1 Validation Test w.r.t. Q094 with OMNISTAR Differencing Enabled

\begin{tabular}{|c|c|c|c|c|}
\hline & Average (m) & Standard Deviation (m) & Average (m) & r.m.s (m) \\
\hline Easting & +0.14 & 0.15 & 0.00 & 0.20 \\
\hline Northing & -0.78 & 0.12 & 0.00 & 0.79 \\
\hline
\end{tabular}

Figure 3.15 clearly shows that the OmniSTAR Differencing technique renders a positional accuracy, well below one meter: half a foot in Easting $(14 \mathrm{~cm})$ and two and a half feet in Northing $(78 \mathrm{~cm})$. The standard deviation in this case reflects the repeatability of the experiment, i.e., the spread around the mean: $15 \mathrm{~cm}$ and $12 \mathrm{~cm}$ in Easting and Northing. The Root Mean Square (r.m.s.) gives a better indication of the accuracy of the recovered position: the spread with respect to the known position of the Q094, part of the Indiana High Accuracy Reference Network (HARN): $20 \mathrm{~cm}$ and $79 \mathrm{~cm}$ for the Easting and Northing, respectively. Figures 3.15 and 3.16 illustrate this feature even better. The $\operatorname{AgGPS}^{\mathrm{TM}} 132$ determined position dwells to the south of the absolute coordinates of Q094 in a small circle. The circle that contains $50 \%$ of the data points is called the Circular Error Probable (CEP). Figure 3.15 reveals that the CEP for this experiment is smaller than $10 \mathrm{~cm}$, a remarkable precise result indeed. The CEP of the absolute position would still be better than 1 meter. The origin of the plot $\{0.00,0.00\}$ in Figure 3.15 is the position of the given absolute reference coordinates of HARN station Q094. 


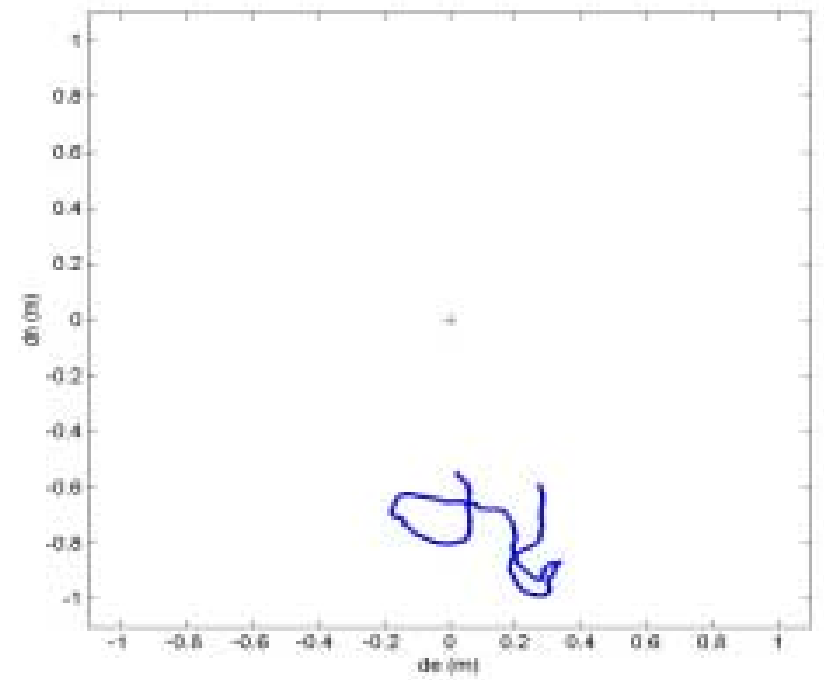

Figure 3.15 GPS Data at Q094 in Differencing Mode
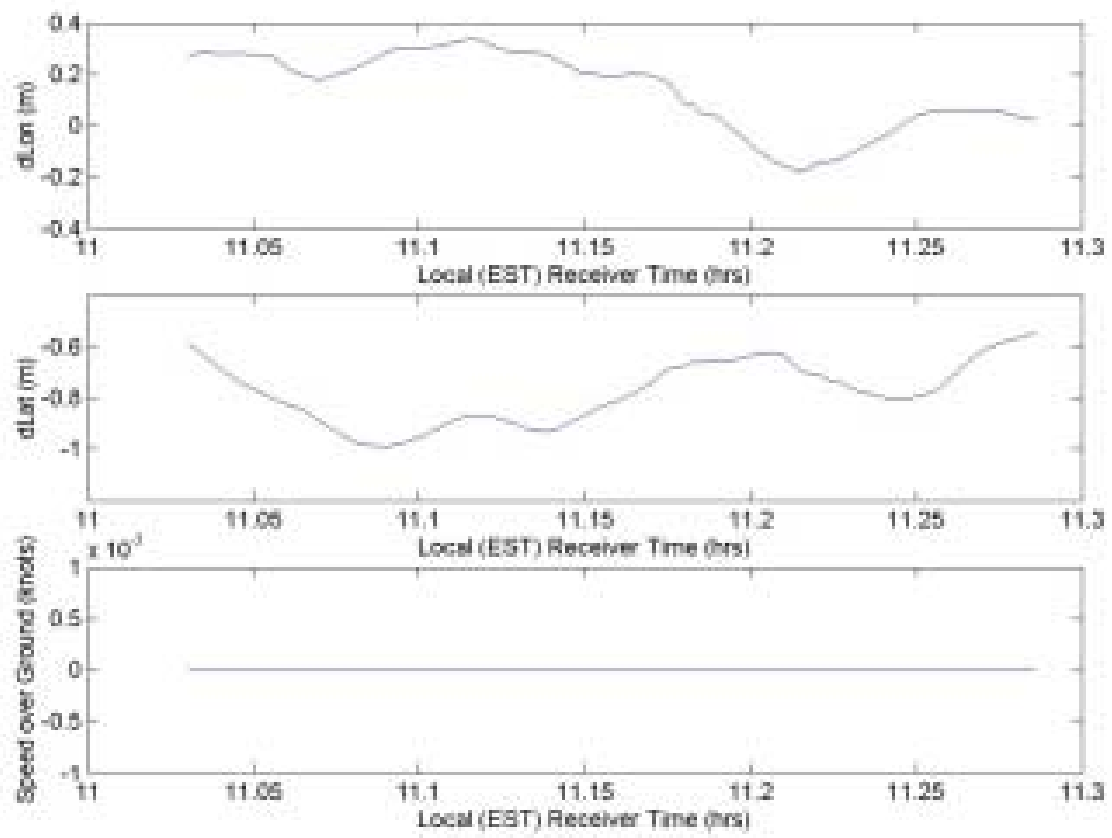

Figure 3.16 Time Histories in Differencing Mode

Figure 3.16 shows the time histories of the longitudinal (EW) (top plot), latitudinal (NS) (middle plot), and velocity (bottom plot) variations. The longitudinal and latitudinal variations stay within half a meter ( $\min$ to $\max$ ). The not-moving receiver 
indeed shows zero velocity with a high degree of accuracy (note that the ordinate axis comprises a $2 * 10^{-3}$ knots interval).

\section{Static Positioning in Undifferenced Mode}

The experiment was repeated minutes later with the OmniSTAR differential capability disabled: between 16:25:06 UTC and 16:40:12 UTC (in local time: between 11:25:06 EST and 11:40:12 EST). The absolute positions of the AgGPS ${ }^{\mathrm{TM}} 132$ over Q094 did not deteriorate to an extent one may expect from a differential service being switched off. Typical parameters as standard deviation and r.m.s. tell only part of the story. The undifferenced location varied over a much larger area (except in longitude) than in the differenced mode. The CEP would give a better description: the CEP of the differenced positions would be around $40 \mathrm{~cm}$, four times worse than the differenced positions, see Figures 3.17 and 3.18. The only variable that agrees with this somewhat increased error is the standard deviation of the point in latitude: $43 \mathrm{~cm}$. See Table 3.2.

Table 3.2 Validation Test w.r.t. Q094. with OmniSTAR Differencing Disabled

\begin{tabular}{|c|c|c|c|c|}
\hline & Average (m) & Standard Deviation (m) & Average (m) & r.m.s. (m) \\
\hline Easting & -0.16 & 0.04 & 0.00 & 0.16 \\
\hline Northing & +0.47 & 0.43 & 0.00 & 0.64 \\
\hline
\end{tabular}

Figure 3.17 clearly reveals the influence of the OMNISTAR Differencing service being switched off. One may suggest that a series of 15 minute experiments need to be conducted, to reach reliable conclusions. However, it should be kept in mind that the GPS accuracy standard is to be assessed for one position fix in a moving vehicle. That means that any one fix of the $\operatorname{AgGPS}^{\mathrm{TM}} 132$-on-the-move may be represented by any one point on the graphs of Figures 3.15 or 3.17. If one introduces the notion of CEP95, i.e., a circle of a certain radius that will obtain all possible position fixes with $95 \%$ probability, than one has to conclude that OmniSTAR differencing technique tends to yield more consistent position fixes than in the undifferenced mode. 


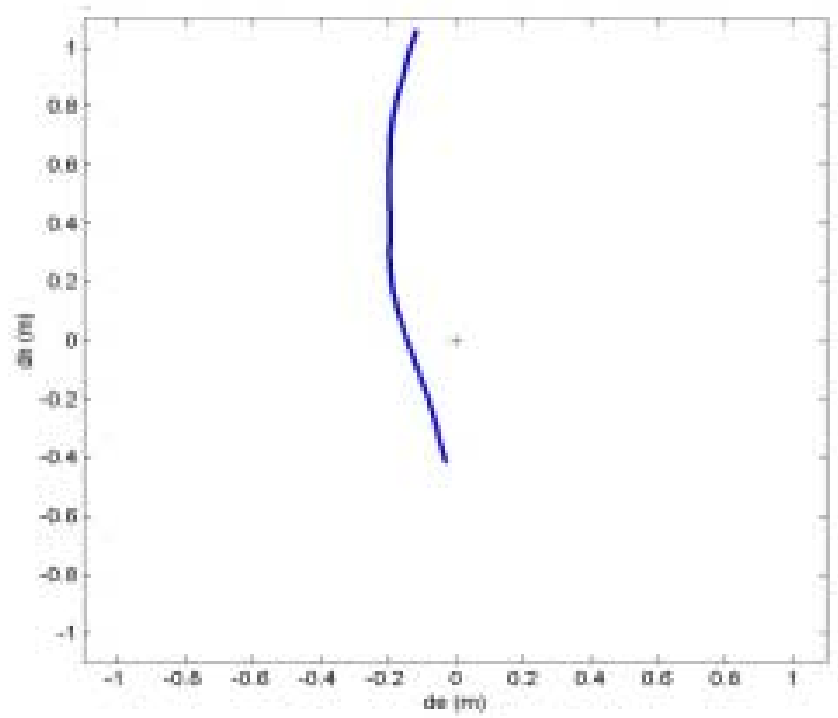

Figure 3.17 GPS Data at Q094 in Undifferencing Mode
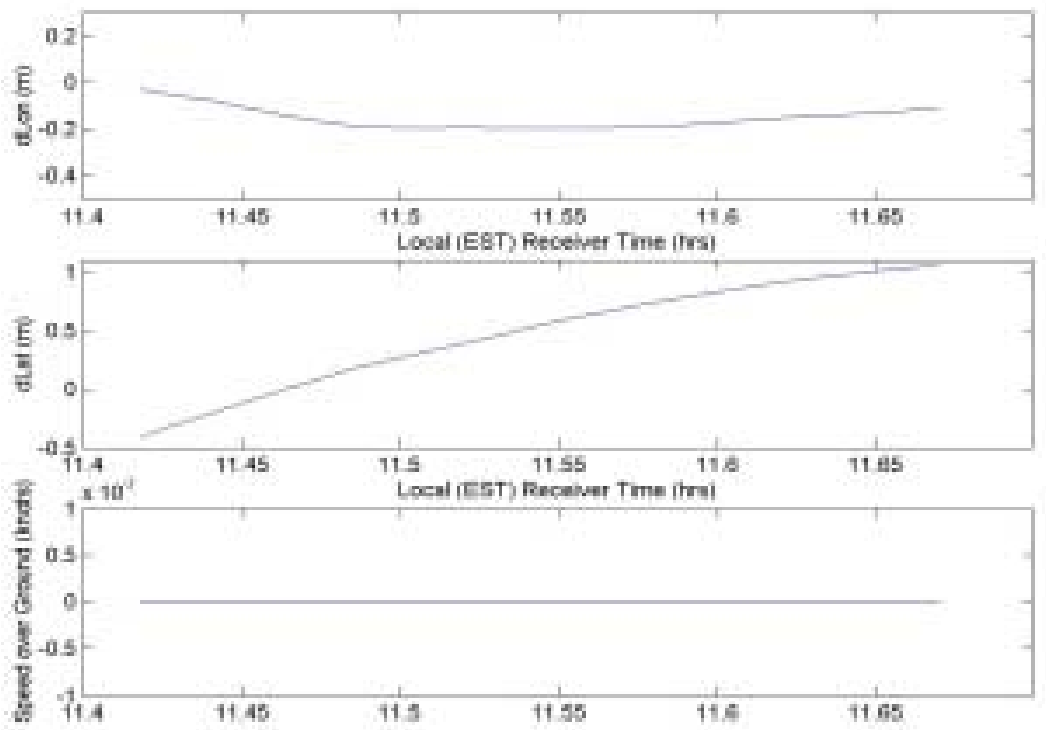

Figure 3.18 Time Histories in Undifferencing Mode

3.5 Verification Tests in June 2000 and January 2002

\section{Verification Test in June 2000}

In June 2000 a test was performed downtown Indianapolis to investigate the effect of high-rise buildings on the antenna signal in an urban canyon. 
Figure 3.19 shows the total trajectory of the test vehicle as intermittent position fixes along its path, Figure 3.19(a), and as a continuous path, Figure 3.19(b), as obtained from GPS. Because of the sometimes erratic behavior of the plot of intermittent fixes, the continuous plot of the car's path is needed to reveal the time sequence between position fixes. However, plotting mere the continuous path would give a too optimistic view of the receiver's capabilities in urban environments. In this case, the intermittent fixes plot gives a good and reliable impression of the "loss-of-lock" circumstances.

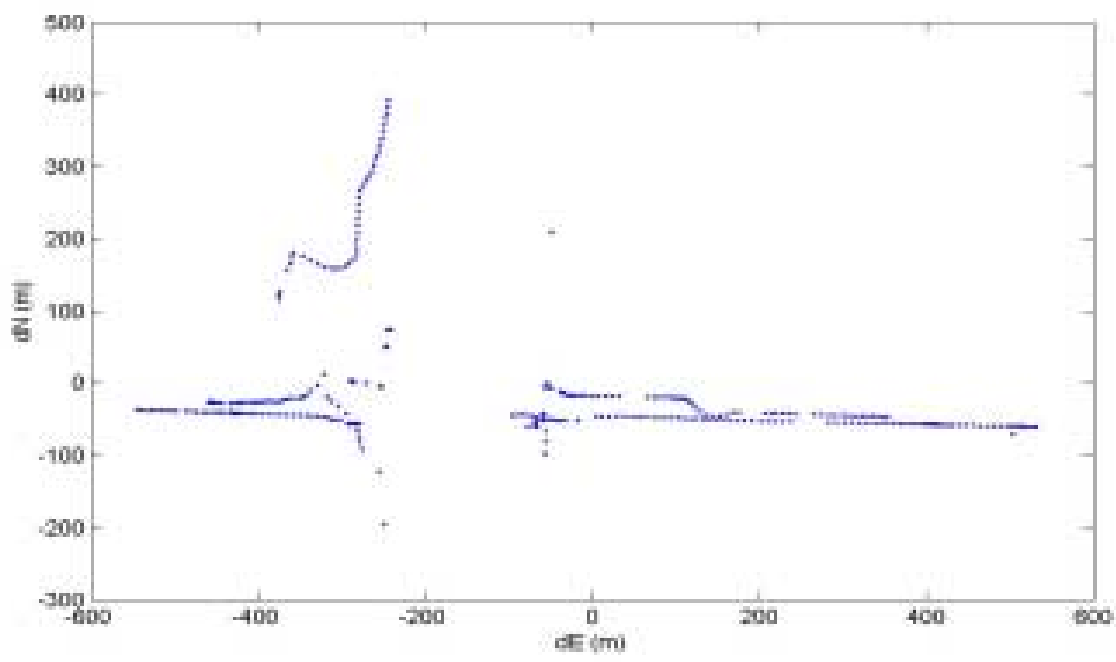

(a) Trajectory as Intermittent Position Fixes

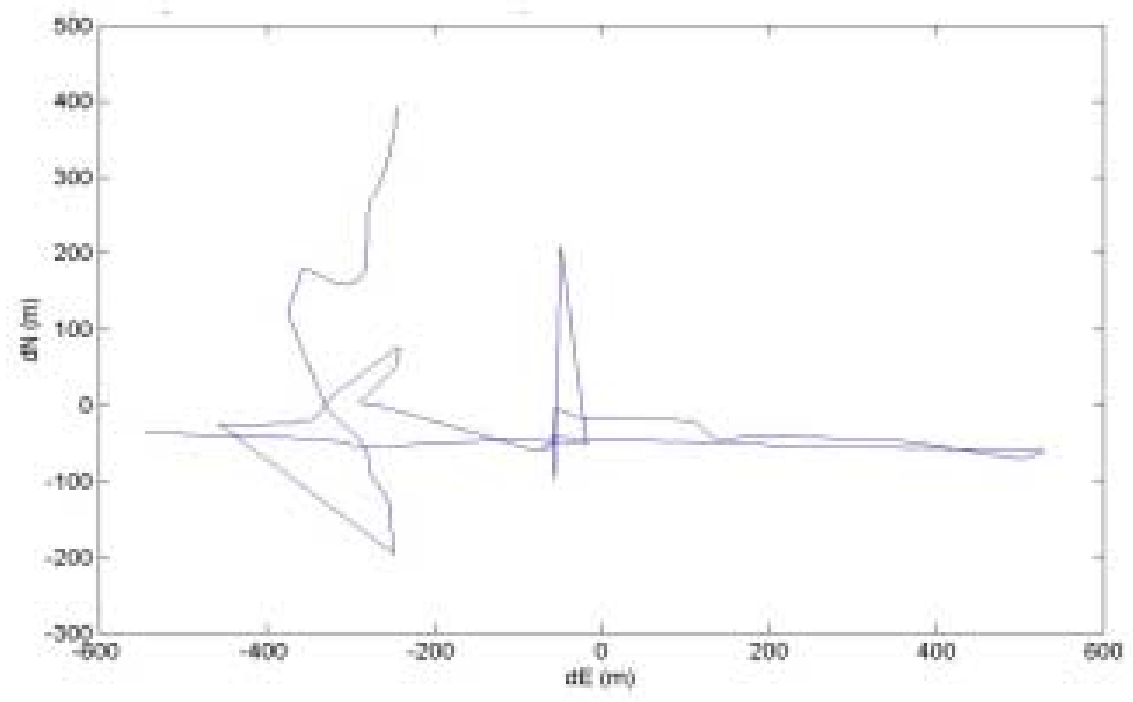

(b) Trajectory as a Continuous Path

Figure 3.19 Trajectory of Test Vehicle 
The next three sets of figures (Figures 3.20, 3.21, and 3.22) show the vehicle trajectories broken up in three distinct segments: first the vehicle is going eastbound on Ohio, as depicted in Figure 3.20. In Figure 3.21, the car has turned around and drives westbound on Ohio (in the opposition direction), and passes the intersection with Illinois. In Figure 3.22, the car after a short distance beyond Illinois has turned around, drives a short distance eastbound on Ohio, and takes a left on Illinois going north. The driven street segments, Ohio and Illinois may be characterized as an "urban canyon", with on the NW and NE corner of the intersection two giant skyscrapers. It is clear from these figures, that along some stretches the position and velocity of the car could be recovered with some acceptable quality. Ohio Street runs east/west, and Illinois street north/south about 250 meters west of the arbitrarily chosen reference position on Ohio Street $\{0,0\}$.
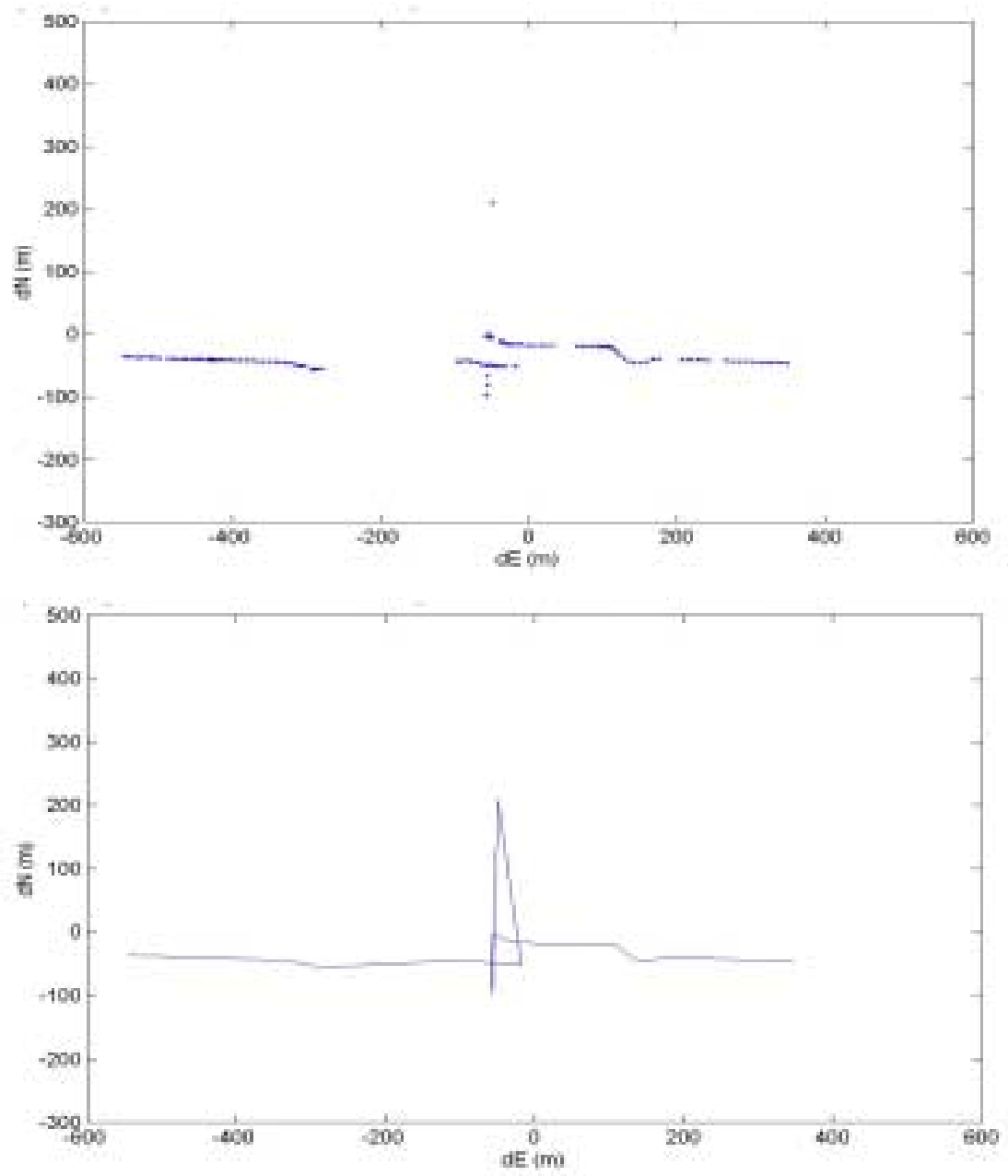

Figure 3.20 Trajectory of Test Vehicle on Ohio Street (Eastbound) 
In Figure 3.20, while the vehicle is going eastbound on Ohio, a large artifact, caused by multipath is clearly visible in the continuous plot: the car appears to go temporarily northbound and southbound around the $\mathrm{dE}=0$ marker. This was not the case in real life.

In Figure 3.21, two things are prominent: data gaps right before the vehicle reaches the intersection with Illinois. This data gap showed also up in Figure 3.20 (intermittent fixes) a while the car was still going eastbound on Ohio. Figure 3.19(a), showing all data, confirms the repeatability of the data gaps in the position fixes around this area. The second item to notice in Figure 3.21 is the apparent erratic behavior of the car, as it just has passed the Illinois intersection with the two large skyscrapers. Around $\mathrm{dE}=-300 \mathrm{~m}$ the car appears to have temporarily driven NE, and subsequently $\mathrm{SW}$, which did not happen in real life.
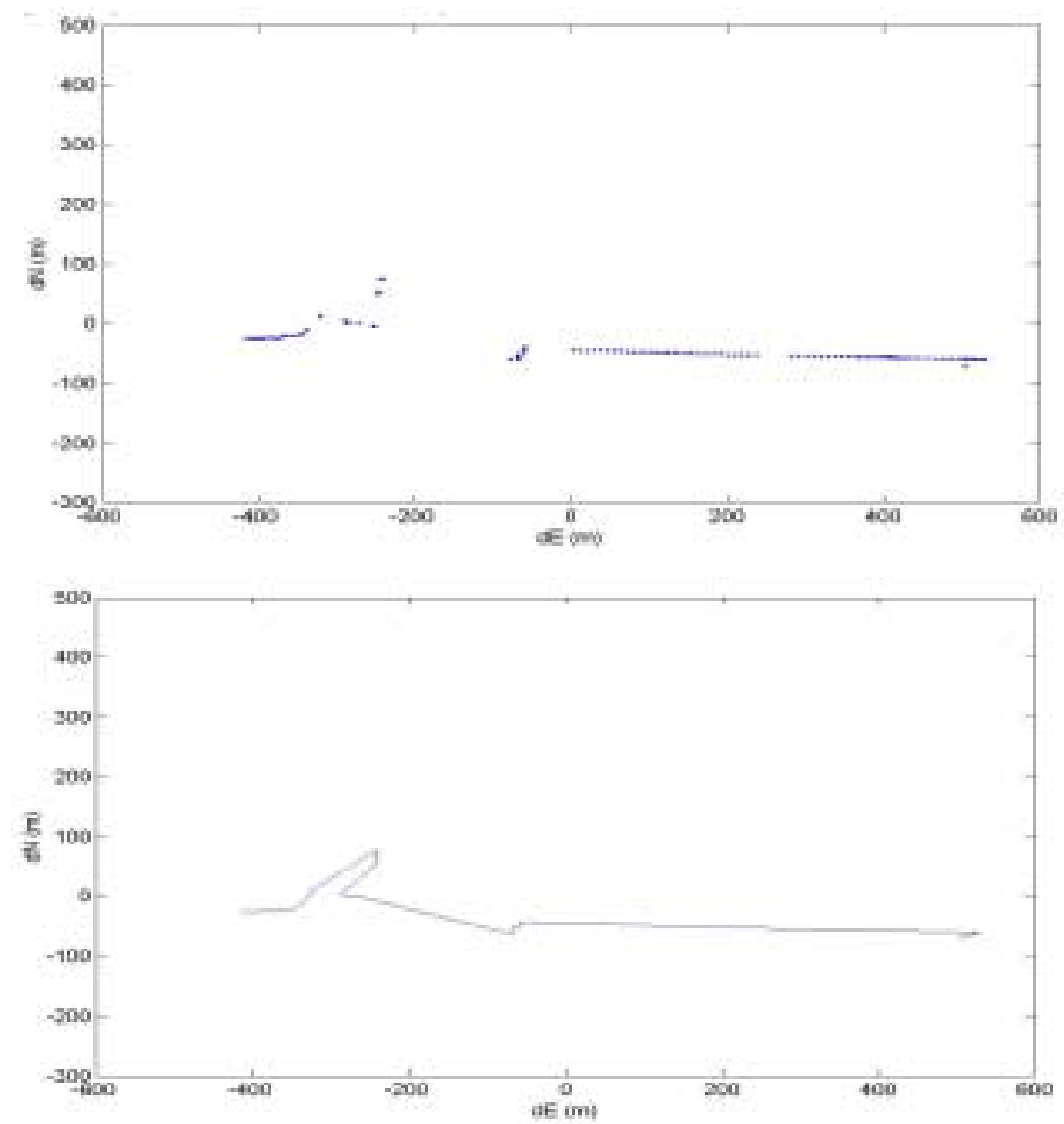

Figure 3.21 Trajectory of Test Vehicle on Ohio Street (Westbound) 
In Figure 3.22, apparent erratic behavior is clearly shown right before the car turns left/north onto Illinois, and right after the car has passed between the two skyscrapers while going north.
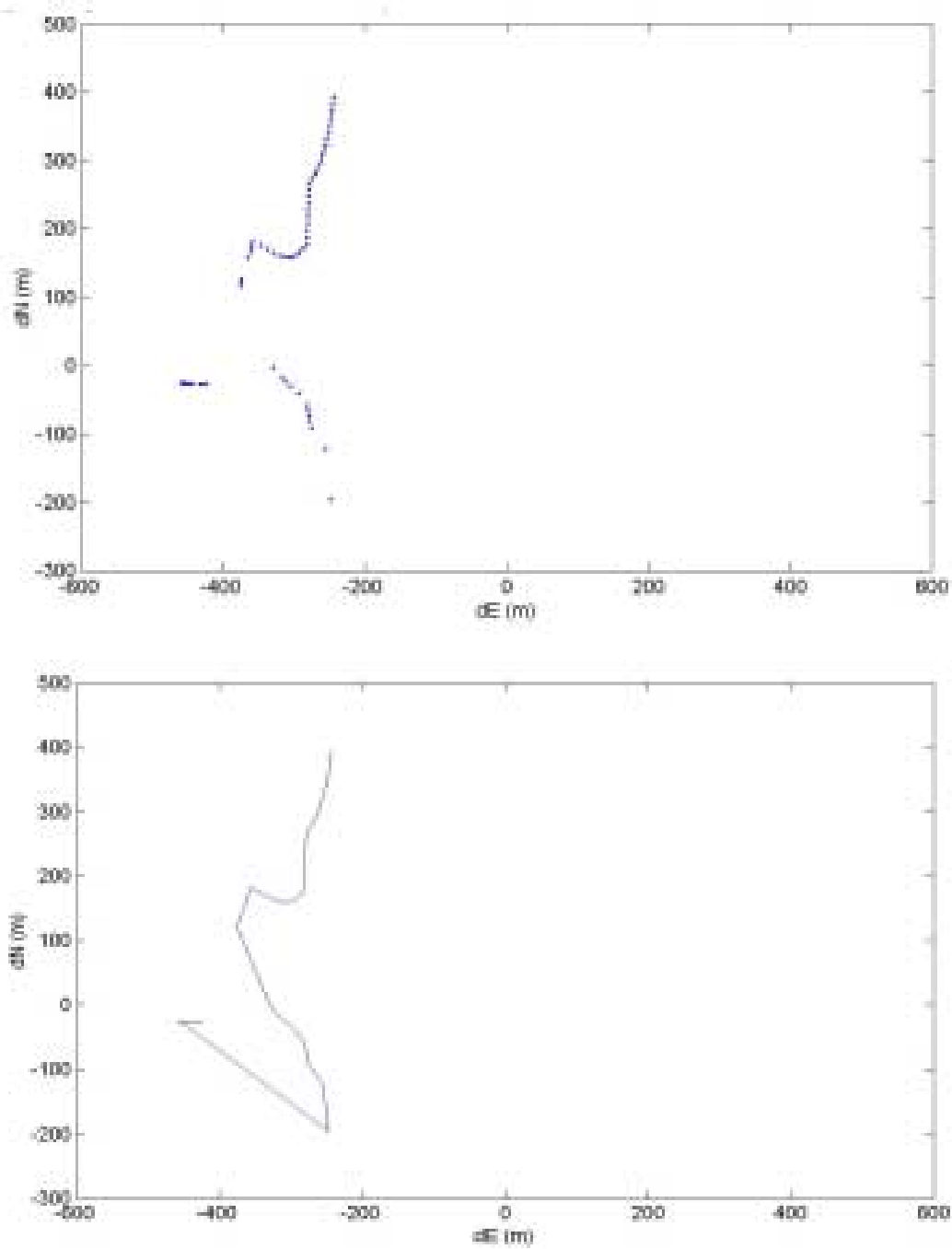

\section{Figure 3.22 Trajectory of Test Vehicle on Illinois Street (Northbound)}

In summary, one may conclude that the majority of the urban route in Indianapolis shows erratic and sporadic behavior. Although we have chosen probably the worst urban scenario in Indiana, the intersection between Ohio and Illinois in downtown Indianapolis, the June 2000 test results lead to the conclusion that car positioning and velocity monitoring is not very feasible in an urban canyon as downtown Indianapolis. 


\section{Verification Test in January 2002}

To confirm these results of June 2000 handheld pseudo-range receivers were brought downtown to see whether pseudo-range solutions could be obtained on the four corners of the intersection of Ohio and Illinois. The NE and the NW corner of the intersection feature each a giant skyscraper. All corners were subsequently visited, and a pseudo-range fix was established. The pseudo-range solution was averaged over 50 position fixes at all four corners, in the following sequence: SE, NE, NW, and SW. The pseudo-range survey was performed in the morning around $10 \mathrm{AM}$, EST, and was repeated in the afternoon around 3:40 PM. Table 3.3 summarizes the positional results in UTM coordinates (zone $16 \mathrm{~S}$ ).

Table 3.3 UTM Coordinates of the Four Corners at the Intersection of Ohio with Illinois (Downtown Indianapolis, January 25, 2002)

\begin{tabular}{|c|c|c|c|}
\hline OHIO-ILLINOIS & Local time EST & East (meters) & North (meters) \\
\hline SE-am & $09: 56$ & 0571983 & 4402541 \\
\hline SE-pm & $15: 38$ & 936 & 577 \\
\hline NE-am & $09: 58$ & 956 & 550 \\
\hline NE-pm & $15: 40$ & 967 & 578 \\
\hline NW-am & $10: 00$ & 948 & 589 \\
\hline NW-pm & $15: 42$ & 988 & 579 \\
\hline SW-am & $10: 04$ & 951 & 558 \\
\hline SW-pm & $15: 45$ & 935 & 580 \\
\hline
\end{tabular}

The coordinates, but more clearly when plotted (see Figure 3.23), show that this intersection is severely handicapped by shielding and multi-path problems. Single-point positioning from a car in motion will most likely fail as well. Notably, see Figure 3.23, the NE-am corner is virtually to the west of the SE-am corner, and to south of the NWam corner, instead of to the north and to the east, respectively. All pm-corners seem to be positioned on a east-west line, with the NW-pm corner to the east of the NE-pm corner. These are all signs of severe signal shielding by buildings, and multi-path problems. 


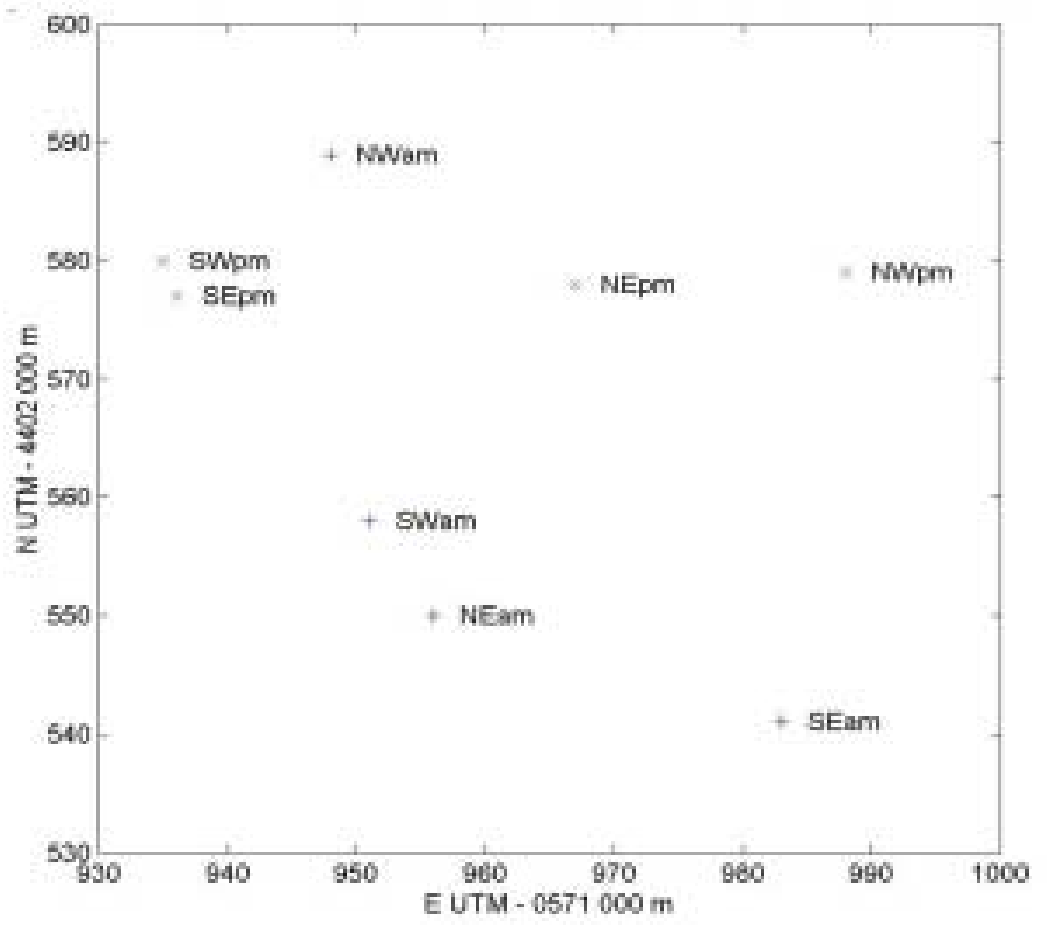

Figure 3.23 Four Corners at the Ohio/Illinois Intersection

\subsection{Summary}

Based on the various validation and verification tests, it may be summarized that

- the AgGPS ${ }^{\mathrm{TM}} 132$ receiver with OmniSTAR differential capability removes errors introduced by Selective Availability to a large extent.

- the $\mathrm{AgGPS}^{\mathrm{TM}} 132$ receiver is capable of position fixes at the level of 1-2 meters in undifferenced mode, once Selective Availability was terminated at midnight between May 1 and 2, 2000.

- The differential service, OmniSTAR, seems still to improve the "SA off" accuracy to the one meter level, or even somewhat better.

- Urban canyons are detrimental to determine reliably a vehicle's position and velocity. 
Chapter 4

\section{DEVELOPMENT OF A COMPUTER PROGRAM FOR DATA COLLECTION AND DATA PROCESSING}

\subsection{Overview of the GPS-Trek Computer Program}

GPS-Trek is a computer program developed for the current study. The GPSTrek program is developed using Microsoft Visual Basic 6.0 and ESRI's MapObjects 2.0 software. The program is designed for Microsoft Windows based platforms. GPS-Trek consists of two separate components: GPS-Trek Data Collection and GPS-Trek Data Processing. The two components can be run independent of each other. Each component has an interactive graphic user interface (GUI). The GUI is designed to be intuitive and user friendly so that minimal training is required for users to run the program. Each GUI has an embedded map component which provides map display and GIS functionality to the program. The GPS-Trek Data Collection component allows users to initiate or stop a data collection session using designated keys on the computer keyboard. Users can monitor the data collection process while the location measured by GPS is displayed on the embedded map in real time. Users can press designated keys during data collection to mark special events. Users also have the option to enter comments at the beginning and/or at the end of a data collection session. The GPS-Trek program automatically records GPS data into a log file during a data collection session. Special marks and comments entered by users are also included in the log file. The GPS-Trek Data Processing component converts data recorded in the GPS log file into a format that allows travel time, distance and speed to be derived and referenced according to map locations. The GPS-Trek program allows locations recorded by GPS to be displayed as points over a base map dynamically in time series. Data recorded in a GPS $\log$ file are represented as a highlighted linear route over the base map. Tools are provided so that users can edit the GPS route interactively on the map. Users can mark special points, such as intersections on the route. Users can also select a section of the GPS route by picking a start and an end point on the route. Users have the option to export the selected 
route as map file or to export the data represented by the selected route to an excel format file for further analysis.

\subsection{Data Collection}

The in-vehicle GPS data collection unit used in the current study consists of two main hardware components, namely, an IBM Thinkpad laptop and an AgGPS ${ }^{\mathrm{TM}} 132$ GPS receiver made by Trimble Navigation. The hardware interfacing between the GPS receiver and the laptop computer is achieved by using the power/data cable included with $\operatorname{AgGPS}^{\mathrm{TM}} 132$ unit. Subscription to OmniSTAR's satellite service enables measurement of position data with real time differential correction. With DGPS, the $\operatorname{AgGPS}^{\mathrm{TM}} 132$ receiver can output sub-meter positions and 0.1 mile-per-hour ( $\mathrm{mph}$ ) velocity accuracy. $\operatorname{AgGPS}^{\mathrm{TM}} 132$ outputs information through NMEA-0183 sentences. NMEA-0183 sentences are strings of comma-delimited text that conform to the standard set by the National Marine Electronics Association. NMEA-0183 sentences always begin with a dollar sign character (\$) followed by a talker ID code and a sentence ID code. Talker ID identifies the source of the data. Sentence ID is used to distinguish the sentence from other NMEA sentences in the data stream. The actual data included in NMEA-0183 sentences is placed in fields, and each field is preceded by a comma character. Different NMEA-0183 sentences have different data fields. The following is an example of an NMEA sentence with a sentence ID RMC:

$\$ \mathrm{GPRMC}, 193901, \mathrm{~A}, 4027.459499, \mathrm{~N}, 08655.562494, \mathrm{~W}, 000.00,0.0,020301,1.9, \mathrm{~W}, \mathrm{D} * 2 \mathrm{E}$

The RMC sentence starts with \$GPRMC. GP is the talker ID which indicates that the data source is GPS. RMC is the sentence ID. Therefore \$GPRMC denotes the start of an RMC sentence transmitted from the GPS device. The string of comma delimited text immediately following \$GPRMC is composed of fields containing the actual data. The first field of an RMC sentence represents the UTC (Universal Time Coordinated) time and are in hhmmss.ss format, where $h \mathrm{~h}$ is hours (from 00-23), $\mathrm{mm}$ is minutes, and ss.ss is seconds and fractions of a second. The second field of an RMC sentence indicates GPS status. 
The value of the second field is either $\mathrm{A}$ or $\mathrm{v}$, where $\mathrm{A}$ means data is valid and $\mathrm{v}$ means the receiver suspects something is wrong. The third and fourth fields of an RMC sentence provide the coordinate of latitude and the direction of the latitude, respectively. Latitude coordinate is given in the format of DDMM.MMMM (with 0-7 decimal places) where $D D$ is degrees, and MM.MMMM is minutes and fractions of a minute. Latitude direction is given as N for North or S for South. Similarly, the fifth and the sixth fields of an RMC sentence provide longitude coordinate and longitude direction, respectively. Longitude coordinate is given in the format of DDDMM.MMMM (with 0-7 decimal places) where $D D D$ is degrees, and MM.MMMM is minutes and fractions of a minute. Longitude direction is given as w for West and E for East. The seventh field of an RMC sentence represents the speed over ground. The unit of the speed is in knots. The eighth field provides track mode in degrees. The ninth field gives the date in ddmmyy format. The tenth field is magnetic variation in degrees. The eleventh field gives the direction of magnetic variation. The twelfth field gives mode indication where A represents Autonomous, D represents Differential, and N indicates that Data is not valid.

The AgGPS ${ }^{\mathrm{TM}} 132$ receiver can generate a selection of NMEA-0183 sentences. The receiver can be configured to output any combination of these NMEA sentences according to user's needs. In traffic studies, traffic engineers are essentially interested in travel time, travel speed, travel distance and traffic delay. All of the above information can be obtained directly or derived from data provided by the NMEA-0183 RMC sentence. Therefore, RMC sentence provides sufficient information for the purpose of current study. To optimize data transmission speed and minimize hard disk usage, the AgGPS receiver is configured to output only one NMEA-0183 sentence, i.e. the RMC sentence.

The GPS-Trek program Data Collection component is installed on the IBM Thinkpad laptop which has hardware interface with the $\mathrm{AgGPS}^{\mathrm{TM}} 132$ receiver. The key function of the data collection component is to receive data transmitted from AgGPS $^{\mathrm{TM}} 132$ via the computer serial port and log the data in a text file. In order for data to be transmitted properly, it is critical that the settings of the serial port match that of the 
GPS receiver output port. In the current study, the input/output parameters are set as following:

$$
\begin{aligned}
& \text { Baud Rate }=9600 \\
& \text { Parity }=0 \\
& \text { Data Bits }=8 \\
& \text { Stop Bits }=1
\end{aligned}
$$

The GPS-Trek program has a graphic user interface that allows users to initiate or stop data collection by pressing specific keys on the computer keyboard. Before data collection starts, user has an option to input some testing information such as road name, direction, starting point or any other comment. The above input will then become headers of the data $\log$ file. A data log file is automatically created by the GPS-Trek program when data collection is initiated. The GPS-Trek program generates the name of log files in the following format: mmddhhmm.raw, where $\mathrm{mm}, \mathrm{dd}$, $h \mathrm{~h}$, and $\mathrm{mm}$ denote the mouth, day, hour and minute of the time when data collection starts, respectively. All log files bear the extension .raw. When a data collection session is initiated, the GPS-Trek program opens the serial communication port and records the incoming NMEA-0183 RMC sentence into the log file. A sample of the log file can be found in Table 4.1.

Table 4.1 A Sample of GPS Log File

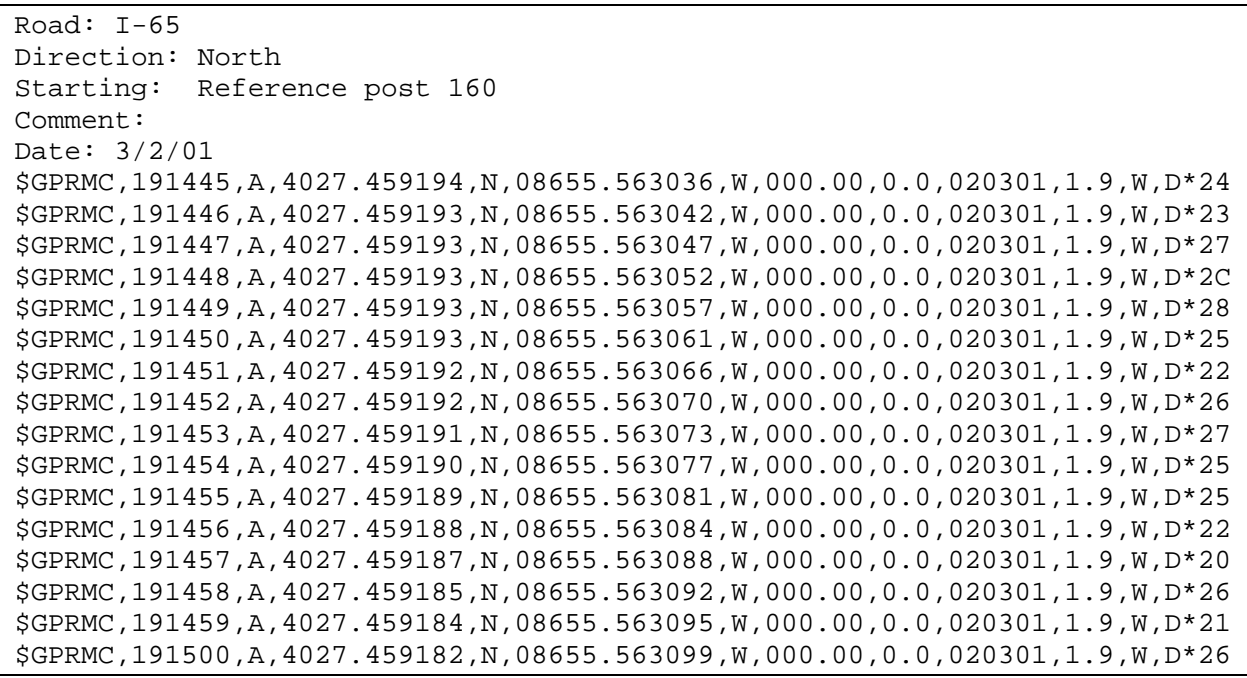


During the data collection, users can press designated keys on the computer keyboard to mark special events. The GPS-Trek program allocates a total number of ten keys on the keyboard for special marking purpose therefore up to 10 types of special events can be marked. When user presses a special marker key, the program generates a "marker" line and appends it in the log file. The following is a sample of a "marker" line:

Marker: 11:25:16 AM 4

The last digit of a "marker" line identifies the marker type.

When a data collection session is being terminated, user has an option to input additional comment if necessary. The comment will then be appended to the log file and the log file will be saved to the hard disk. All log files are placed in the $<$ Data $>$ subfolder under the main program folder.

The user interface of the GPS-Trek Data Collection program contains an embedded map component. A real-time tracking option is available in the program that allows the position obtained by the GPS receiver to be displayed as map points and is updated in real time. This allows the user to monitor the data that are being collected. Real time tracking options can be disabled or enabled through a menu in the user interface.

By default, the AgGPS ${ }^{\mathrm{TM}} 132$ receiver outputs data at a rate of $1 \mathrm{~Hz}$. The receiver can be configured to run at higher data rate such as $5 \mathrm{~Hz}$ or $10 \mathrm{~Hz}$. We have found that the $1 \mathrm{~Hz}$ data rate is sufficient for the purpose of the current study.

Although the default settings of the GPS receiver for the current study is to output only the NMEA-0183 RMC sentence at $1 \mathrm{~Hz}$ data rate, the GPS-Trek program can handle GPS output with any combinations of NMEA-0183 sentences. The program can also handle data collection at different data rate. Due to the use of an event driven mechanism, data reception at different rate and with different sentence content is 
automatically handled by the program without any requirement for user intervention. However, we have found that with an IBM Thinkpad laptop running at $300 \mathrm{MHz}$ processing speed and at GPS data output rate of $5 \mathrm{~Hz}$ or $10 \mathrm{~Hz}$, the performance of real time map tracking feature are deteriorated. Therefore, the real time tracking option should be disabled during data collection at higher than $1 \mathrm{~Hz}$ rate.

The size of the GPS log file is an important parameter for determining the data storage requirement. We have estimated that, at a data rate of $1 \mathrm{~Hz}$ and with $\mathrm{RMC}$ sentence output, the size of a log file containing one hour's GPS data is about 270 kilo bytes. In another word, it takes less than $3 \mathrm{Mb}$ of hard disk space to save all the information obtained during ten hours of continuous data collection. Therefore, with computer hard drive space running at Gigbytes level nowadays, data storage is not a major concern for the current study.

\subsection{Data Conversion}

As mentioned above, the GPS log file generated during data collection process contains the NMEA RMC sentences. RMC sentences contain information of longitude, latitude, time and speed. A data conversion process is necessary to derive information such as location, travel time, distance and speed from the elements in RMC sentences.

\section{Conversion of UTC Time to Local Time}

GPS time is recorded as Coordinated Universal Time (UTC), which is conventionally referred to as the Greenwich Meridian Time (GMT). Zero (0) hours UTC is midnight in Greenwich England, which lies on the zero longitudinal meridian. Universal time is based on a 24 hour clock. The world is split into 15 degree wide longitudinal bands (360 degree / 24 hours). Each band represents one hour. In the Indiana time zone (East), the local time lags the UTC time by five hours. Therefore in current study, local time is obtained using the following equation:

Local time (in hhmmss) $=$ UTC time (in hhmmss) -50000 . 


\section{Calculation of Travel Time}

Travel time at any point is calculated as the difference between the recorded time at the target point and the starting point. Recorded time is first converted to seconds from the hhmmss format using the following equation:

$\mathrm{t}_{\mathrm{s}}=3600 h h+60 m m+s s$

where $t_{s}$ represents time in seconds.

\section{Calculation of Travel Distance}

Distance traveled between two consecutive recorded GPS points are calculated using the following equation:

$D_{i, i-1}=\sqrt{\left(X_{u t m}^{i}-X_{u t m}^{i-1}\right)^{2}+\left(Y_{u t m}^{i}-Y_{u t m}^{i-1}\right)^{2}}$

where $\mathrm{D}_{\mathrm{i}, \mathrm{i}-1}$ is the distance between point $\mathrm{i}$ and point $\mathrm{i}-1$, and $\left(X_{u t m}^{i}, Y_{u t m}^{i}\right)$ and $\left(X_{u t m}^{i-1}, Y_{u t m}^{i-1}\right)$ are the UTM coordinates of points and point $\mathrm{i}-1$, respectively.

Travel distance between any two points are calculated by summation of distances of all consecutive GPS points in between the two target points, as shown in the following equation:

$\mathrm{D}_{\mathrm{i}, \mathrm{j}}=\mathrm{D}_{\mathrm{i}, \mathrm{i}-1}+\mathrm{D}_{\mathrm{i}-1, \mathrm{i}-2}+\ldots \ldots+\mathrm{D}_{\mathrm{j}+1, j}$

where $D_{i, j}$ is the distance between point $i$ and point $j$, and $i>j$

\section{Conversion of Longitude and Latitude to UTM Coordinate}

The GPS receiver outputs the location of a point using longitude and latitude. The value of longitude and latitude is based on a standard global reference system called 
World Geodetic System 1984 (WGS 84) (1). WGS 84 was adopted as a world standard from a datum called the North American Datum of 1983 (NAD 83). For all practical purposes there is no difference between WGS 84 and NAD 83. In the WGS 84 system, longitude and latitude provide coordinates of a point on an ellipsoid surface. When the location of the point needs to be represented on a map, a process called map projection is necessary to relate the coordinates of longitude and latitude to coordinates on a plane surface. A map projection is a systematic representation of a part of the earth's surface on a plane. Different types of map projections have been developed to minimize map distortions, depending on the intended use of the map. The most widely used map projection is Universal Transverse Mercator (UTM), which is also used in the current study. The procedures involved in transformation of WGS 84 geodetic coordinates (latitude, longitude) to UTM coordinates (X, Y) can be found in the Handbook for Transformation of Datums, Projections, Grids and Common Coordinates Systems published by the Topographic Engineering Center of U.S. Army Corps of Engineers (5). Given a point with geodetic coordinates $(\phi, \lambda)$ in radians, where $\phi$ is the latitude and $\lambda$ is longitude, the Transverse Mercator coordinates $\left(\mathrm{x}_{\mathrm{TM}}, \mathrm{y}_{\mathrm{TM}}\right)$ can be obtained from the following equation:

$$
\begin{aligned}
& \mathrm{x}_{\mathrm{TM}}=\mathrm{N} \Lambda \cos \phi+\frac{\mathrm{N} \Lambda^{3} \cos ^{3} \phi}{6}\left(1-\mathrm{t}^{2}+\eta^{2}\right) \\
& +\frac{\mathrm{N} \Lambda^{5} \cos ^{5} \phi}{120}\left(5-18 \mathrm{t}^{2}+\mathrm{t}^{4}+14 \eta^{2}-58 \mathrm{t}^{2} \eta^{2}\right) \\
& \mathrm{y}_{\mathrm{TM}}=\mathrm{S}_{\phi}+\frac{\mathrm{N} \Lambda^{2}}{2} \sin \phi \cos \phi \\
& +\frac{\mathrm{N} \Lambda^{4}}{24} \sin \phi \cos ^{3} \phi\left(5-\mathrm{t}^{2}+9 \eta^{2}+4 \eta^{4}\right) \\
& +\frac{\mathrm{N} \Lambda^{6}}{720} \sin \phi \cos ^{5} \phi\left(61-58 \mathrm{t}^{2}+\mathrm{t}^{4}+270 \eta^{2}-330 \mathrm{t}^{2} \eta^{2}\right)
\end{aligned}
$$

where $\mathrm{N}=\frac{\mathrm{a}}{\sqrt{1-\varepsilon^{2} \sin ^{2} \phi}}$ 


$$
\begin{aligned}
& \mathrm{t}=\tan \phi \\
& \eta=\varepsilon^{\prime} \cos \phi \\
& \varepsilon^{\prime 2}=\frac{\varepsilon^{2}}{\left(1-\varepsilon^{2}\right)} \\
& \varepsilon^{2}=\frac{\mathrm{a}^{2}-\mathrm{b}^{2}}{\mathrm{a}^{2}} \\
& \mathrm{~S}_{\phi}=\mathrm{a}\left[\mathrm{A}_{0} \phi-\mathrm{A}_{2} \sin 2 \phi+\mathrm{A}_{4} \sin 4 \phi-\mathrm{A}_{6} \sin 6 \phi+\mathrm{A}_{8} \sin 8 \phi\right] \\
& \mathrm{A}_{0}=1-\frac{1}{4} \varepsilon^{2}-\frac{3}{64} \varepsilon^{4}-\frac{5}{256} \varepsilon^{6}-\frac{175}{16384} \varepsilon^{8} \\
& \mathrm{~A}_{2}=\frac{3}{8}\left(\varepsilon^{2}+\frac{1}{4} \varepsilon^{4}+\frac{15}{128} \varepsilon^{6}-\frac{455}{4096} \varepsilon^{8}\right) \\
& \mathrm{A}_{4}=\frac{15}{256}\left(\varepsilon^{4}+\frac{3}{4} \varepsilon^{6}-\frac{77}{128} \varepsilon^{8}\right) \\
& \mathrm{A}_{6}=\frac{35}{3072}\left(\varepsilon^{6}-\frac{41}{32} \varepsilon^{8}\right) \\
& \mathrm{A}_{8}=-\frac{315}{131072} \varepsilon^{8} \\
& \Lambda=\lambda-\lambda \text { in radians }
\end{aligned}
$$

$\lambda_{0}$ is central meridian in radians

$$
\lambda_{0}= \begin{cases}\frac{(6 Z-183) \pi}{180} & (\mathrm{Z} \geq 31) \\ \frac{(6 \mathrm{Z}+177) \pi}{180} & (\mathrm{Z} \leq 30)\end{cases}
$$

$\mathrm{Z}$ is UTM zone

$$
Z= \begin{cases}\text { Greatest Integer } \leq\left(31+\frac{\lambda}{6}\right) & (0 \leq \lambda<\pi) \\ \text { Greatest Integer }<=\left(\frac{\lambda}{6}-29\right) & (\pi \leq \lambda<2 \pi)\end{cases}
$$


$\mathrm{a}$ and $\mathrm{b}$ are the semi-major axis and semi-minor axis for WGS 84 reference ellipsoid, respectively.

$$
\begin{aligned}
& a=6378137 m \\
& b=6356752.3142 m
\end{aligned}
$$

The UTM coordinates ( $\mathrm{X}_{\mathrm{UTM}}, \mathrm{Y}_{\mathrm{UTM}}$ ) can be obtained from the following equation:

$\mathrm{X}_{\mathrm{UTM}}=0.9996 \mathrm{x}_{\mathrm{TM}}+500,000$

$\mathrm{Y}_{\mathrm{UTM}}=0.9996 \mathrm{y}_{\mathrm{TM}}$

\section{Calculation of Travel Speed}

The speed output from the RMC sentence is in the unit of knots. To convert the speed from knots to mile per hour (mph), the following equation is used:

\section{$1 \mathrm{knot}=1.15 \mathrm{mph}$}

\section{$\underline{\text { File Conversion }}$}

Data conversion is handled by File Converter tool in the GPS-Trek Data Processing program. The input file is the GPS log file (.raw file) generated during the data collection process. The user needs to specify an output file name. The default output file name extension is .gps. A sample of the .gps file can be found in Table 4.2. The .gps files are text files in ASCII format. The .gps files inherit the headers from the corresponding .raw files. Following the headers are the converted GPS data, with each line representing a GPS data point originated from an RMC sentence. Ten data fields are reported for each data point, which include date, time, UTM coordinate X, Y in meters, UTM zone number, travel speed in $\mathrm{mph}$, travel distance in meter, and travel time in seconds. The Marker field reports markers made during data collection. As described in 
3.2, the value of markers ranges from 1 to 10 , with different values representing different user designated events. A space in the marker field means no markers are made for the specific data point. The last data field is intersection. This field can be used to mark the intersection points on the test road, which is of great interest in traffic analysis. The GPSTrek Data Processing program provides tools to mark intersection points after data is collected. A detailed description of this utility can be found in the following section. A marked intersection point is represented by a value of $\mathbf{0}$ in the Intersection field.

Table 4.2 A Sample of the .gps File

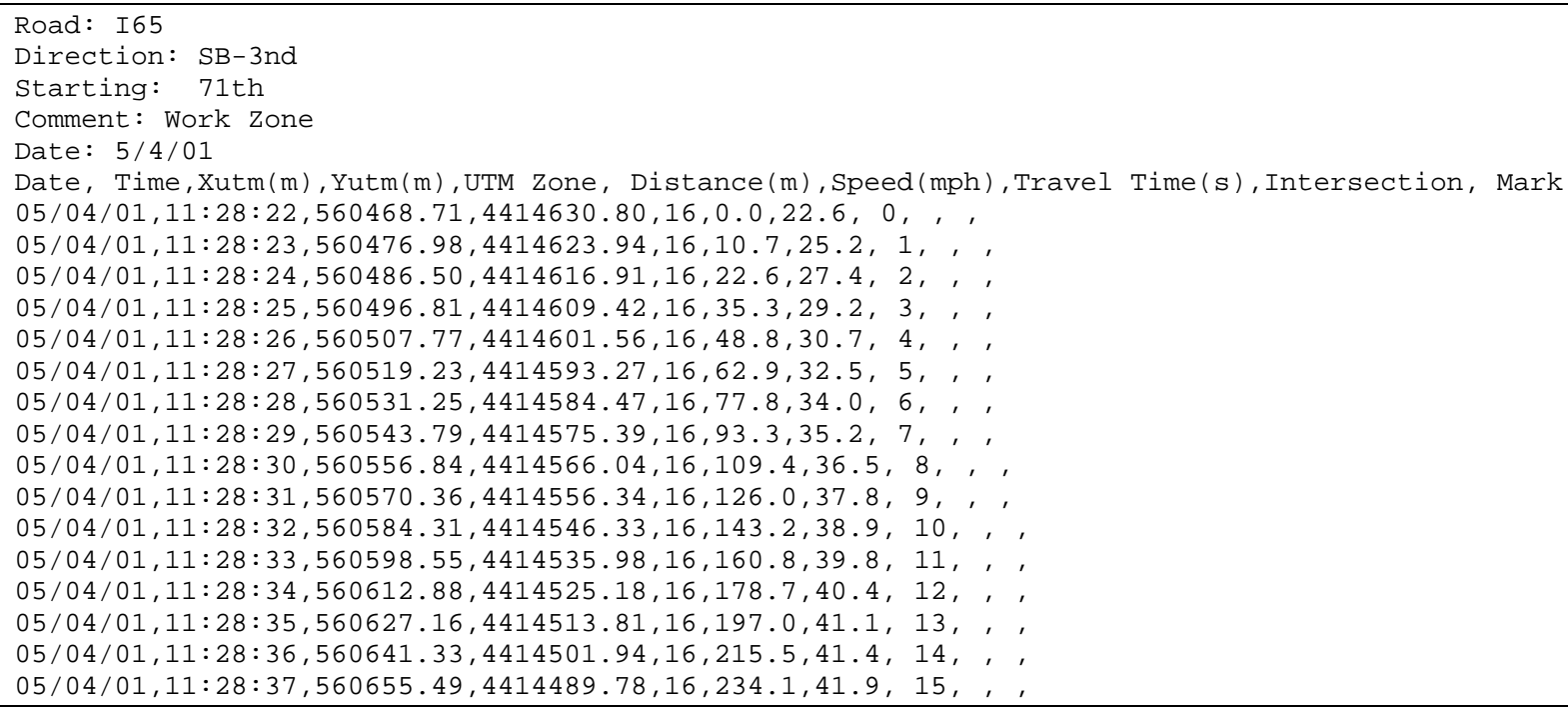

\subsection{Data Processing and Data Management}

After file conversion, each GPS data point contained in a gps file can be displayed as a location on a map according to its UTM coordinates. The GPS-Trek Data Processing component allows the GPS data to be displayed and manipulated in the format of map points. In general, the GPS-Trek Data Processing component provides the following functions:

- Allow GPS data to be presented dynamically on a base map in time sequence.

- Allow selective viewing of any segment of GPS data.

- Allow insertion of intersection marks to the GPS data. 
- Allow selection of any segment of GPS data and export the data in Microsoft ${ }^{\circledR}$ Excel format worksheet.

- Export a sequence of GPS location into a map file.

Details of these key features are discussed in the following sections.

\section{$\underline{\text { Map Component }}$}

A base map is needed in order to properly reference the location represented by GPS data points. The default base map in GPS-Trek Data Analysis program is an Indiana highway map which contains the Interstate, US and State routes within the Indiana boundary. Additional map layers can be added to the base map using the Add Map Content tool provided by the GPS-Trek program. In order for the map content to be added successfully, the map files need to be in the right format. The GPS-Trek program recognizes maps in ESRI's shapefile format. Map projection is critical for data referencing. All map files used in the GPS-Trek program should use UTM and the NAD 83 datum.

\section{GPS Data Display}

Individual GPS data points are displayed as points on the base map. A sequence of GPS data points are represented as route in the format of lines. The GPS-Trek program can read in a .gps file and display the GPS data points contained in the file on the map sequentially in time series. This allows the user to view the GPS data as if the data is being collected in real time. Users can view the change of location on the map while time and speed associated with the corresponding location are being displayed in the program window. After the entire file content is played, the trace of the GPS location is highlighted as a linear route. Tools are provided by the GPS-Trek program to allow users to select any data point using a selection cursor, and view the location, speed and time associate with the specific point. Tools are also provided for users to select any segment of the route and replay the corresponding data sequence. Users can pause and resume the dynamic display at any point. 


\section{Insertion of Intersection Marks}

Identification of intersection location is of great importance in traffic flow analysis. Intersection locations can be marked in the field by using the tool provided in the data collection program of GPS-Trek program. However, this process requires constant operator intervention and is error prone. Safety concern is another issue if data collection is performed by one operator who is also driving the vehicle. Due to the aforementioned problems, we recommend against marking intersections during field data collection. Instead, we have designed a tool that allows the intersection location to be identified and marked during the data analysis process in the GPS-Trek program. The intersection marking tool works in the following steps:

- A base map that includes the intersection(s) of interest should be present.

- The GPS data segments of interest have been played and the GPS route has been highlighted on the map.

- The user should drag the selection cursor near an intersection point as referenced by the base map. The selection cursor can be moved one GPS point at a time with the speed associated with each point being displayed. The user can then try to identify an intersection point by moving the cursor while observing the speed change associated with the GPS points.

- Once an intersection point is identified, the user can use the Mark as Intersection tool to mark the point on the base map.

\section{Selection and Exporting of the GPS Data}

It is conceivable that sometimes a user is only interested in certain portion of the GPS data contained in a .gps file. Therefore, we provide a tool in the GPS-Trek Data Processing Program to allow users to select only the data that are of interest to them. To select a segment of GPS data, the user can use a mouse to drag the selection cursor to the starting point of the GPS segment, and mark the point using the Mark as Start tool. Then the user can drag the selection cursor to the end point of the GPS segment, and mark the

point using the Mark as End tool. Once the starting and end points of the GPS segments are defined, the user can export the selection to an external file. The user can choose to 
export the file in a comma delimited text format or an excel worksheet format. The Excel file contains data of the selected segments. Data fields of the Excel files are the same as that of the .gps files, as shown in Table 4.2. The Excel files contain essential information for traffic studies such as travel time, travel speed and travel distance and intersection location. These data can be readily analyzed and charted using the built-in Excel functions. 
Chapter 5

\section{SAMPLE SIZE REQUIREMENTS AND FIELD PROCEDURE FOR GPS TRAFFIC DATA COLLECTION}

As described in the preceding chapters, the GPS devices provide the users with the fundamental data in terms of latitude, longitude, elevation and UTC time. Based on these spatial and temporal data, traffic engineers can determine the most useful traffic information, including travel time, travel speed, travel distance and delay. Therefore, the GPS data collection is one of the most important tasks for traffic engineers to perform transportation studies utilizing the GPS devices. Similar to the existing methods like the average vehicle and moving vehicle methods, the GPS traffic data can be collected in peak hours or off-peak hours, depending on the purpose of the study. Because the fundamental GPS data may vary with traffic volume, traffic composition, traffic control devices and weather, the resulting traffic data vary from day to day and time to time. To produce reliable traffic information from the GPS data, it is of significance to meet the sample size requirements and follow an appropriate field procedure.

\subsection{Sample Size Requirements}

\section{Current Methods for Estimating Minimum Sample Size}

A large sample is always anticipated to provide us with more information about the population. As the sample size increases, the data will become more representative of the real world traffic conditions and the traffic engineers will be more confident for their analysis and results. On the other hand, the cost for data collection will increase in terms of time and manpower. For example, this study has collected GPS data on SR26 in downtown Lafayette, Indiana. The road segment is about 2.0 miles $(3,276 \mathrm{~m})$ long with eleven signals. It took about three and half-hours to complete 24 test runs (12 for each direction), and a one-way trip approximately took 9 minutes. Therefore, determination of sample size is also a trade-off between the required accuracy and the cost. 
The Institute of Transportation Engineers (ITE) has provided a method for estimating the minimum sample size for travel time and delay studies along a roadway segment below (6):

$\mathrm{R}_{\mathrm{ITE}}=\frac{\sum \mathrm{A}}{\mathrm{N}-1}$

where $R_{\text {ITE }}$ is the average range in running speed, $\Sigma A$ is the sum of calculated speed difference, and $\mathrm{N}$ is the number of completed test runs.

To estimate the minimum sample size, it is required to perform 2 to 4 initial test runs so as to calculate the average range in running speed, $\mathrm{R}_{\mathrm{ITE}}$. After $\mathrm{R}_{\mathrm{ITE}}$ is determined, the minimum sample size with $95 \%$ confidence can be found in Table 5.1, according to the permitted error. Quiroga et al. have examined the ITE method (7). They concluded that this method contains systematic numerical errors and may result in lower than expected sample sizes. To correct the ITE formulation, Quiroga et al. provided a socalled hybrid method for estimating the minimum sample size as follows:

Table 5.1 Minimum Sample-Size Requirements for Travel-Time and Delay Studies

\begin{tabular}{|c|c|c|c|c|c|}
\hline \multirow{2}{*}{$\begin{array}{c}\text { Average Range in } \\
\text { Running Speed, km/h }\end{array}$} & \multicolumn{5}{|c|}{ Minimum Number of Runs for a permitted Error of: } \\
\cline { 2 - 6 } & $2.0 \mathrm{~km} / \mathrm{h}$ & $3.5 \mathrm{~km} / \mathrm{h}$ & $5.0 \mathrm{~km} / \mathrm{h}$ & $6.5 \mathrm{~km} / \mathrm{h}$ & $8.0 \mathrm{~km} / \mathrm{h}$ \\
\hline 5.0 & 4 & 3 & 2 & 2 & 2 \\
\hline 10.0 & 8 & 4 & 3 & 3 & 2 \\
\hline 15.0 & 14 & 7 & 5 & 3 & 2 \\
\hline 20.0 & 21 & 9 & 6 & 5 & 4 \\
\hline 25.0 & 28 & 13 & 8 & 6 & 5 \\
\hline 30.0 & 38 & 16 & 10 & 7 & 6 \\
\hline
\end{tabular}

(Source: ITE Manual of Transportation Engineering Studies)

$\mathrm{n}=\left[\frac{\mathrm{t}_{\alpha} \overline{\mathrm{R}}}{\mathrm{d} \varepsilon}\right]^{2}$ 
where $\alpha$ is the significance level, $\mathrm{t}_{\alpha}$ is the $\mathrm{t}$-value for two-tailed distribution statistics with n-1 degrees of freedom for a confidence level of 1- $\alpha, \varepsilon$ is the user-selected allowable error in the estimate of the mean speed, $d$ is the ratio of $|\bar{R}|$ to $\sigma$ (standard deviation of the population), and $\bar{R}$ is the sample range based on available data, which is computed using the equation below

$$
\overline{\mathrm{R}}=\max _{i=1}^{m} v_{i}-\min _{i=1}^{m} v_{i}
$$

where $\mathrm{m}$ is the sample size available, and $\mathrm{v}_{\mathrm{i}}$ is the ith segment speed observation of the initial study.

To estimate a parameter of the population under investigation, such as the population mean, the sample statistics, like the sample mean, should satisfy the following relationship (8)

$$
\overline{\mathrm{X}}=\frac{\mu}{1-\frac{\mathrm{CV}}{\sqrt{\mathrm{N}}} \mathrm{t}_{\alpha / 2}}
$$

where $\bar{X}$ is the sample mean (estimator), $\mu$ is the population mean (true value), $N$ is the sample size, $\mathrm{CV}$ is the coefficient of variation in the sample, $\alpha$ is the significance level, and $t_{\alpha / 2}$ is the $t$-value of the two-tailed t-distribution with a confidence level of 1- $\alpha$.

It is demonstrated that in Eq. 5.4, estimation of a parameter of the population depends on the selected significance, $\alpha$, sample variation and sample size. As $\alpha$ decreases, the justification of rejecting hypothesis $\mathrm{H}_{0}$ (Type I error) becomes stronger and the probability of committing Type I error decreases. However, for many real life problems, the consequence of favoring alternative hypothesis $\mathrm{H}_{\mathrm{a}}$ (Type II error) is more serious and it is advisable to pick a large value of $\alpha$. For most general engineering problems, the widely used $\alpha$ value is 0.05 . Also, it is noted that with a large sample size, 
the probabilities of committing both types of errors can be reduced. The minimum sample size can be obtained by rewriting Eq. 5.4 as

$\mathrm{N}=\left(\frac{\overline{\mathrm{X}} \times \mathrm{CV} \times \mathrm{t}_{\alpha / 2}}{\overline{\mathrm{X}}-\mu}\right)^{2}$

Letting $\sigma=\overline{\mathrm{X}} \times C V$ and $\varepsilon=\overline{\mathrm{X}}-\mu$, the above equation can be reduced into

$\mathrm{N}=\left(\frac{\sigma_{\alpha / 2}}{\varepsilon}\right)^{2}$

where $\sigma$ is the population standard deviation, and $\varepsilon=$ permitted error.

To the authors' knowledge, both the ITE method and the method formulated by Quiroga et al. are derived on the basis of Eq. 5.6. As an illustration, Eq. 5.2 will become Eq. 5.6 when substituting $d=|\bar{R}| / \sigma$ into Eq. 5.2. Because determination of the $t_{\alpha / 2}$ values involves estimating the number of the degrees of freedom, it is a common practice to use the $\mathrm{Z}$-value of the standard normal distribution, $Z_{\alpha / 2}$, to approximate $t_{\alpha / 2}$. Such an approximation will generate some errors. However, this is the most common practice in the area of civil engineering and the resulting errors should be tolerable. Figure 5.1 shows the discrepancies between t-value and Z-value for $90 \%$ and $95 \%$ confidences, respectively. The $\mathrm{Z}$-value is independent of sample size, and nevertheless, the t-value varies with the sample size. It is demonstrated that the t-value is always greater than the corresponding Z-value. The largest discrepancy occurs when the number of the degrees of freedom, $(\mathrm{N}-1)$, equals one, i.e. the sample size, $\mathrm{N}$, equals two. If the sample size grows to three, the discrepancy drops significantly. After the sample size is greater than five, the discrepancy becomes negligible. This implies that the sample size should not be less than three. It is recommended that if possible, a minimum sample size of five for $90 \%$ confidence or six for $95 \%$ confidence should be considered so as to reduce the potential errors in estimation of the t-value. 


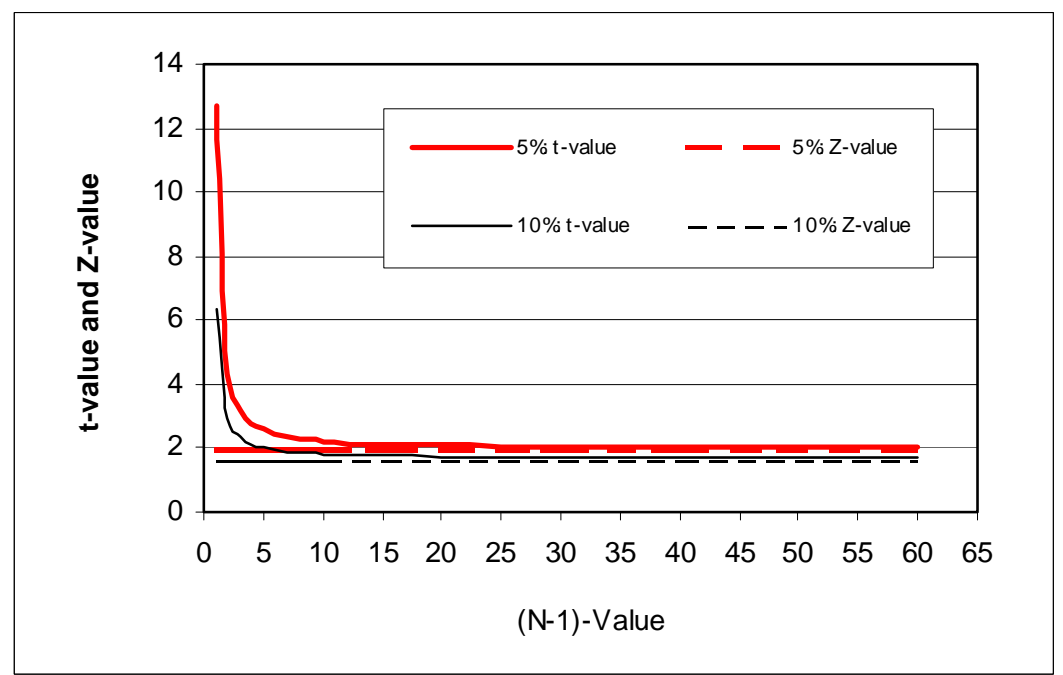

Figure 5.1 Variations of t-Value and Z-value with Sample Size

\section{Estimates of Dispersion}

Some other potential errors may also arise in the estimate of the population standard deviation, $\sigma$. The standard deviation is a measure of dispersion or variability. Likewise, the range and the mean deviation can also be used to measure the dispersion. In statistics, the range and the mean deviation are defined below, respectively

$\mathrm{R}=\operatorname{Max}(\mathrm{X})-\operatorname{Min}(\mathrm{X})$

where $\mathrm{R}$ is the range, i.e. the difference between the largest and smallest values, and $\mathrm{X}$ is the data set.

$\overline{\mathrm{D}}=\frac{\sum\left|\mathrm{x}_{\mathrm{i}}-\overline{\mathrm{x}}\right|}{\mathrm{N}}$

where $\bar{D}$ is the mean deviation, $x_{i}$ is the individual value, $\bar{x}$ is the sample mean, and $N$ is the sample size. 
The range is the simplest measure of the dispersion. Once the largest and smallest values are picked, the range can be easily computed. Since the range depends only on two extreme values and ignores the in-between values, it may not provide a full picture of the dispersion. There exist many cases where the dispersion will be overestimated because of the presence of an unusually extreme value. As a result, the range has been considered as the least satisfactory of all measures of dispersion. It is indicated that in Eq. 5.8, however, the mean deviation takes into account all values in the sample in contrast with the range. Since the arithmetic mean is the most important measure of central tendency, the mean deviation is the arithmetic mean of the absolute deviations from the mean, $\bar{x}$, and the mean deviation is a better measure of the dispersion than the range. This was confirmed by the travel time and speed measurements made on SR26 in downtown Lafayette, Indiana, as shown in Figure 5.2.

Figure 5.2 gives all measures of dispersion in travel time and travel speed. The bold solid line denotes, the range, R, from Eq. 5.7, the bold dashed line the ITE range, $\mathrm{R}_{\mathrm{ITE}}$, from Eq. 5.1, the thin solid line the sample standard deviation, $\mathrm{s}$, and the thin dashed line the mean deviation, $\bar{D}$, from Eq. 5.8. It is shown that the variations of $R_{I T E}$, $\overline{\mathrm{D}}$ and s vary with a similar trend and become stable as the number of initial test runs increases, except for the range, $\mathrm{R}$. However, the ITE range, $\mathrm{R}_{\mathrm{ITE}}$, is greater than the sample standard deviation, s, and may undergo larger variation. The reason is that in the process of computing $\mathrm{R}_{\mathrm{ITE}}$, as shown in Eq. 5.1, greater weights are given to the intermediate values between the first and last observations, resulting in biased estimate. Discrepancy also exists between the mean deviation, $\overline{\mathrm{D}}$, and the sample standard deviation, $\sigma$. It is also observed that because of the tenth observation, the values of the range, $\mathrm{R}$, for both travel time and travel speed experienced significant rise. Notice that this significant rise is probably independent of the number of available data and depends to great extent on the presence of the extreme values. As a result, the use of any estimate of the standard deviation will generate errors in the process of determining the minimum sample size. 

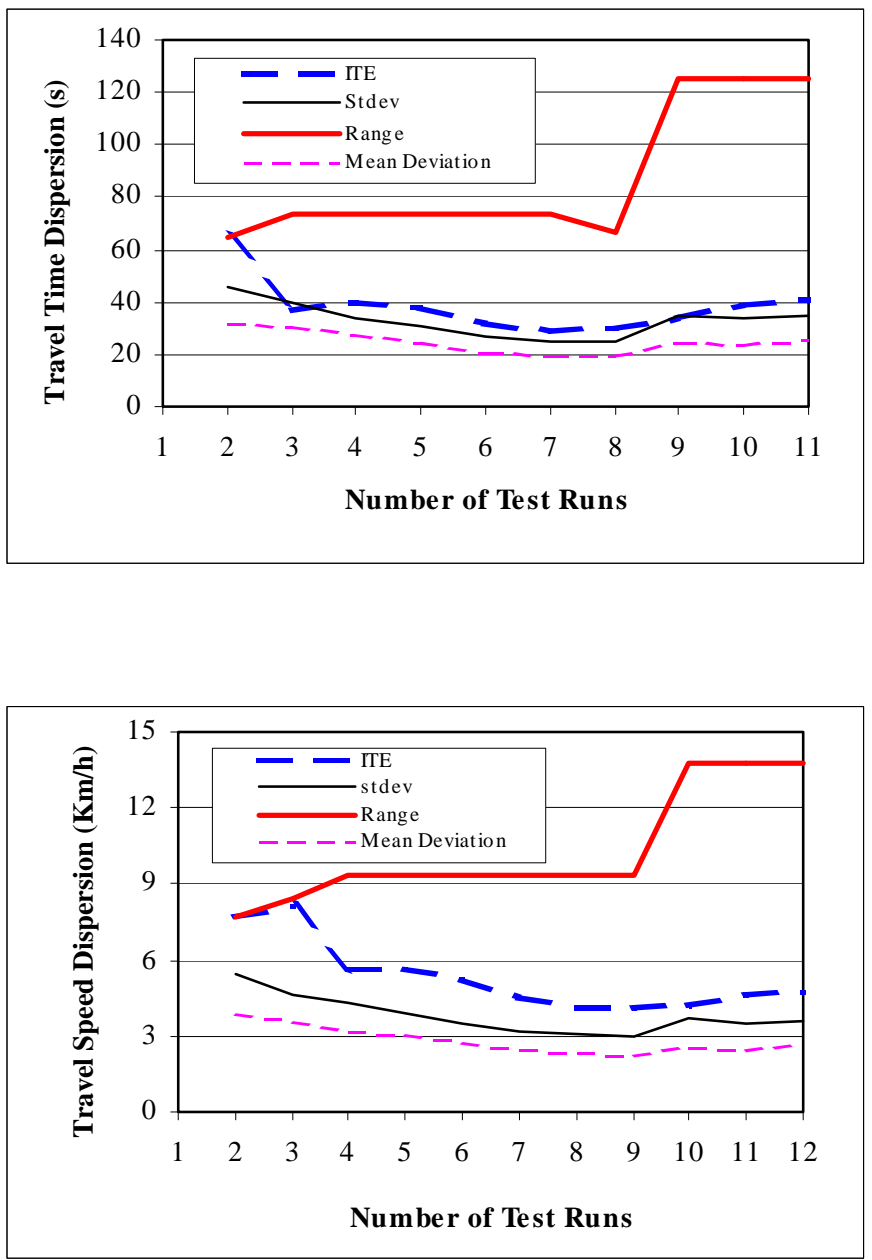

Figure 5.2 Travel Time and Speed Dispersions

Figure 5.3 presents the minimum sample sizes computed using the ITE method, the so-called hybrid method and Eq. 5.6, on the basis of various initial test runs. The values computed from Eq. 5.6 using t-value can be considered as the accurate values. It is demonstrated that the hybrid method produces the largest value and the ITE method the smallest value. The hybrid method employs an iteration algorithm to calculate the sample size requirement with respect to the actual t-value instead of Z-value. However, such an effort is lessened since the hybrid method uses the range to estimate the dispersion, resulting in inflated dispersion. Consequently, the minimum sample size may be overestimated by the hybrid method. The sample size computed using the ITE method or Eq. 5.6 approaches a stable number as the initial test runs grow. This is sound in 
statistics. Nevertheless, the ITE method is based on a biased estimate of the dispersion and usually underestimates the sample size. Therefore, it is advisable to use Eq. 5.6, rather than the ITE method or the hybrid method, to determine the minimum sample size and avoid any biased estimate.

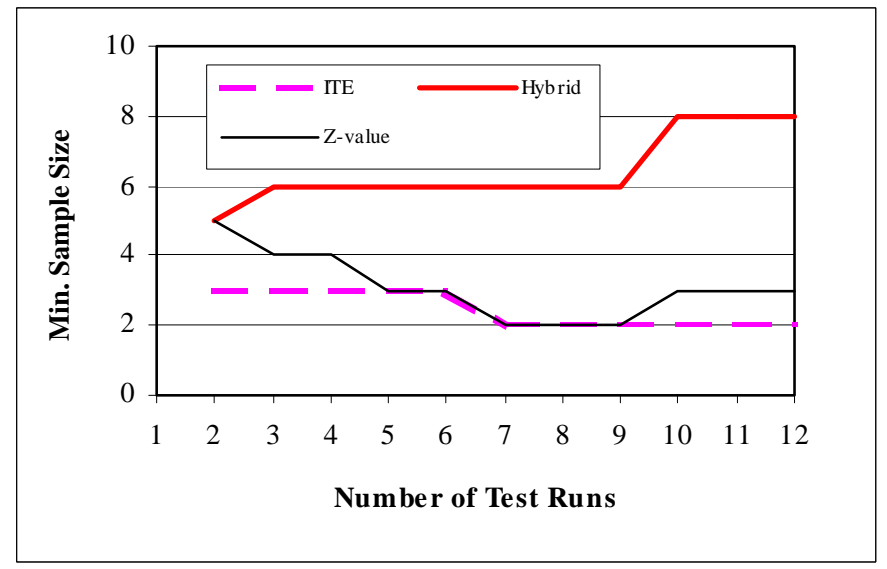

Figure 5.3 Minimum Sample Size Computed Using Various Method

\section{Modified Equation for Determining Minimum Sample Size}

As discussed previously, the use of t-value causes considerable problems mathematically since the t-value depends on the sample size and iteration must be performed to search the possible sample size. As a common practice, the Z-value is employed in place of the t-value. This treatment results in errors and usually generates lower values than the t-value does. However, such an error is predictable as shown in Figure 5.4. In Figure 5.4, the minimum sample sizes are computed using both t-value and Z-value for a selected level of confidence, $90 \%, 95 \%$ and $99 \%$, respectively. It is likely that the errors are independent of the sample size and vary only with the selected confidence level. As the confidence increases, the error grows slightly. Based on the numerical results, it is recommended that the minimum sample size should be determined using the equation below

$\mathrm{N}=\left(\frac{\sigma \mathrm{Z}_{\alpha / 2}}{\varepsilon}\right)^{2}+\varepsilon_{\mathrm{N}}$ 
where $\varepsilon_{N}$ is the sample size adjustment and the other variables are as defined earlier. The $\varepsilon_{\mathrm{N}}$ and $\mathrm{Z}_{\alpha / 2}$ values are given in Table 5.2.

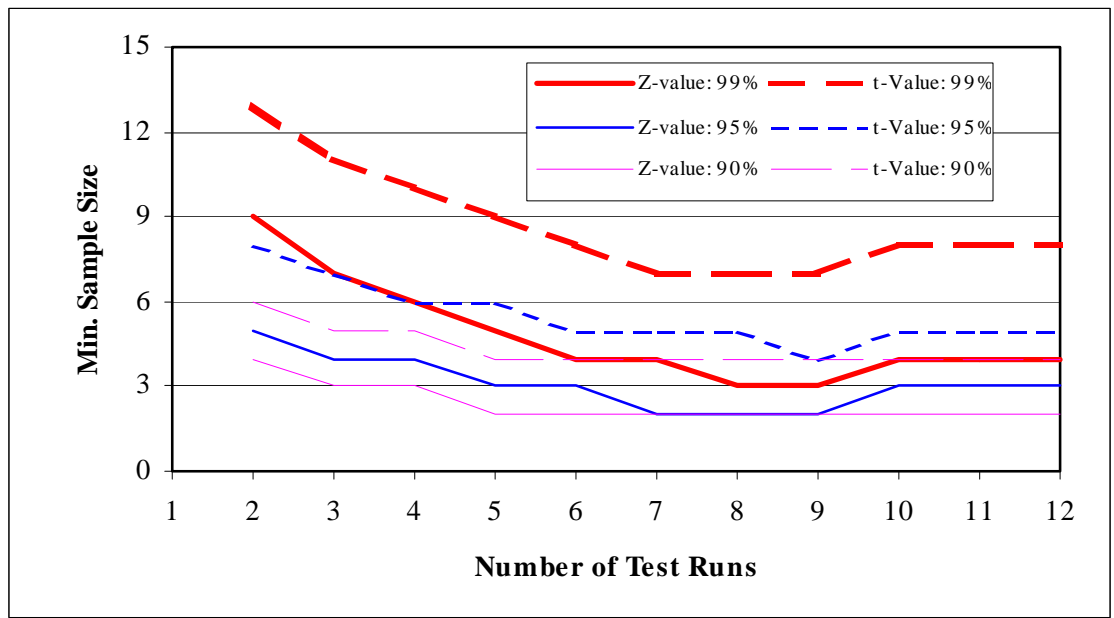

Figure 5.4 Minimum Sample Size Computed Using t-Value and Z-Value

Table 5.2 Values of $\varepsilon_{\mathrm{N}}$ and $\mathrm{Z}_{\alpha / 2}$ for Determining Sample Size

\begin{tabular}{|c|c|c|}
\hline Level of Confidence, $\alpha$ & $\boldsymbol{\varepsilon}_{\mathbf{N}}$ & $\mathbf{Z}_{\boldsymbol{\alpha} / \mathbf{2}}$ \\
\hline $90 \%$ & 2 & 1.64 \\
\hline $95 \%$ & 3 & 1.96 \\
\hline $99 \%$ & 4 & 2.58 \\
\hline
\end{tabular}

\subsection{Field Procedure}

\section{Installation of GPS Data Collection System}

An appropriate field procedure is required for GPS traffic data collection. Following the appropriate procedure, the operator can save time, avoid potential hardware/software problems, and enhance safety. Figure 5.5 shows a test car installed with all devices necessary for GPS traffic data collections, as shown in Figure 5.6. The $\operatorname{AgGPS}^{\mathrm{TM}} 132$, including the antenna and receiver, is used to receive satellite signal and output GPS position data. The external devices include a laptop computer with the GPS- 
Trek and power supply devices. The laptop computer is employed to communicate with the GPS receiver and store the GPS position data.

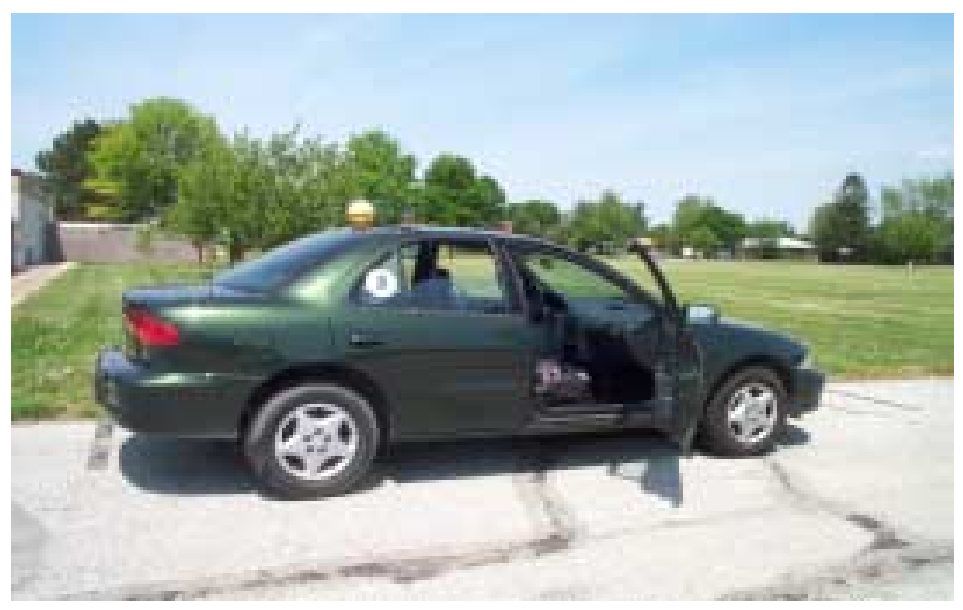

Figure 5.5 Test Car for GPS Traffic Data Collection

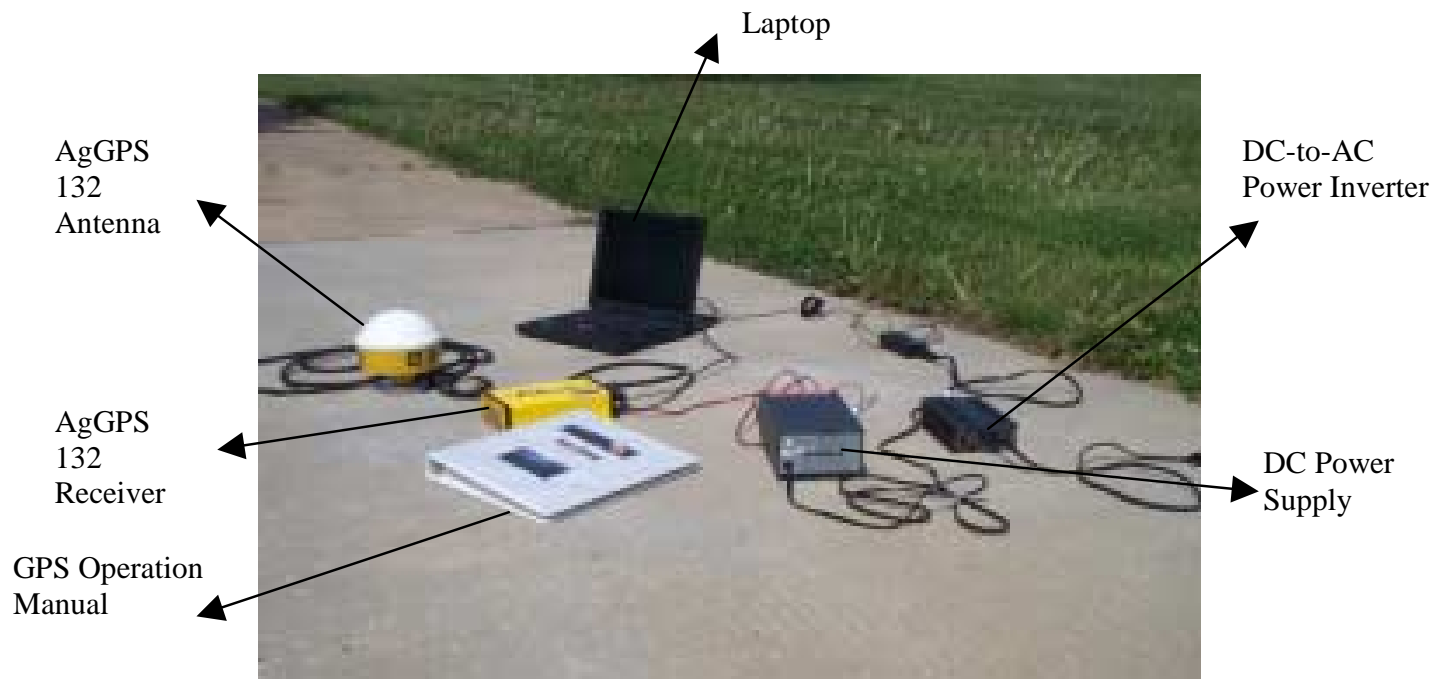

Figure 5.6 GPS and External Devices

The DC-to-AC power inverter is used to convert the direct current (DC) to the alternating current (AC) electricity. The DC power is the standard power you can find from the cigarette lighter socket in a vehicle that provides 13.8 Volts when the vehicle engine is running. The AC power is the $120 \mathrm{~V}$ standard power for household appliances 
and is used here to run the laptop computer. The DC power supply is utilized to convert the AC electricity back to the DC electricity that is required to run the GPS receivers. It should be noted that if the laptop computer is run by other power sources, such as batteries, the DC-to-AC power inverter is not required and the DC power supply may be connected to the vehicle cigarette lighter socket using a vehicle DC power cord.

It is of great importance that before driving to the test road, the operator should install the GPS and external devices properly, rather than on site. Before installation, the operator should always read the safety instructions for the GPS devices, laptop computer, power inverter and DC power supply, so as to reduce the risk of electric shock, fire and damage to the devices. The installation of the devices should follow the steps below:

- Turn off all devices requiring power.

- Set the DC-to-AC power inverter switch to OFF.

- Set the DC power supply switch to OFF and connect the DC power supply to one of the two AC outlets on the DC-to-AC power inverter.

- Connect the laptop computer to the other AC outlet on the DC-to-AC power inverter using the power cord and AC adapter.

- Connect the GPS receiver to the DC power supply. First, turn the sleeves of the two binding output posts (one is red and the other black) counterclockwise to expose the wire wholes. Secondly, insert the positive wire (usually red) of the Data/Power cable provided by the GPS manufacturer into the red binding post's wire hole and the negative wire (usually black) of the Data/Power cable into the black binding post's wire hole. Finally, tighten the sleeves to secure the wire in place. Finally, insert and tighten the sleeve.

- Connect the CONXALL right-angle connector of the Data/Power cable in step 5 to one of the two ports (usually Port A on the GPS receiver.

- Connect the 9-pin DE-9 Female connector to the 9-pin DE-9 Male connector on the laptop computer. 
- Connect the GPS antenna to the GPS receiver using the Ruggedized Antenna Cable. The 90-degree connector leads to the antenna and the straight connector to the receiver.

- Mount the antenna on a flat surface of the test car using the magnetic mount provided by the GPS manufacturer. Make sure the surface is flat and put one piece of paper between the magnetic mount and the selected surface so as to avoid of scratch on the surface.

- Start vehicle engine and connect the vehicle DC power cord on the DC-to$\mathrm{AC}$ power inverter to the cigarette lighter socket on the vehicle.

- Set all devices' switches to ON.

It should be noted that there are many types of power supply devices that are commercially available. Different devices may have different features. Therefore, the installation of the GPS data collection system really depends on the devices you have and may differ from the procedures as described above.

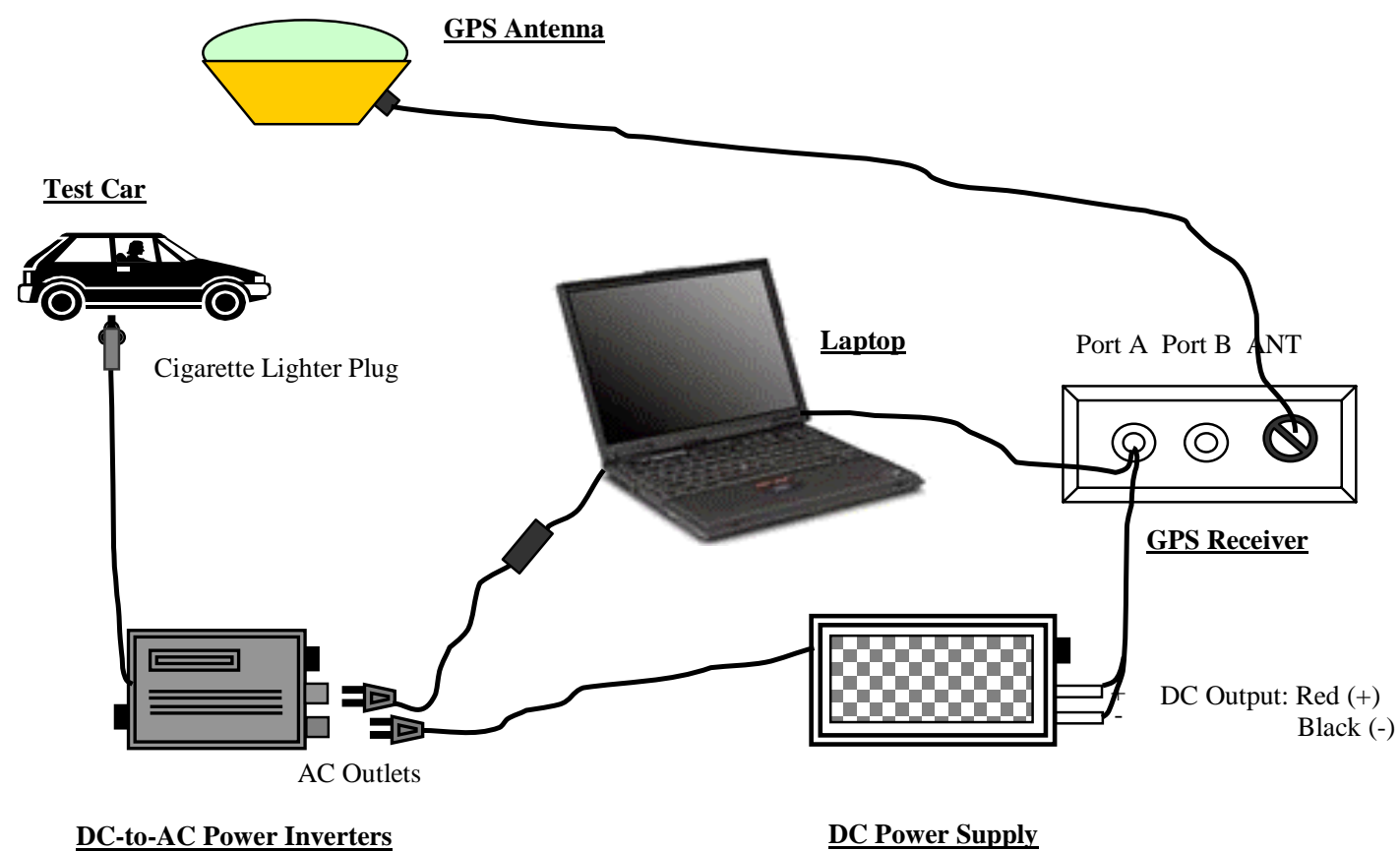

Figure 5.7 Installation of GPS and External Devices 


\section{Field Data Collection}

The operator must take necessary training to conduct field data collection using the above system, especially the GPS receiver and the GPS-Trek program. While the GPS-Trek is developed so that the operator is not required to specify the start point and the end point on the route, it is always recommended that the operator should review the route and the locations of interest before the data collection begins. One operator (driver) can perform field data collection safely in most cases. However, safety concern may arise in some special studies, such as work zone study, in which the operator may be required to mark some special points within a work zone, including the start and end points, offramp, on-ramp and the positions of the taper. In these special situations, the data collection requires two operators.

After the test car is started, the operator can connect the DC-to-AC power inverter to the cigarette lighter receptacle on the car, and then, set the power switches on the power inverter and DC power supply to ON. Once the GPS receiver is powered, its front panel displays the Home screen and the satellite DGPS or GPS information, such as GPS and DGPS status indicators, appears on the screen. The operator should make sure that all indicators satisfy the requirements, i.e. the number of satellites used should be at least 5 (for sub-meter accuracy) and the signal-to-noise ratio greater than 6. Other requirements refer to the manual for a specific GPS device. If the GPS devices function well, the operator may turn on the laptop computer and test the GPS-Trek program. Start the GPS-Trek program and have a trial of data collection. Open the data file and make sure that the program works properly. Table 5.3 shows the format of the raw data file created during the data collection.

The operator can start to collect GPS position data any time before he reaches the test route and complete the required test runs continuously. As a result, all data are saved in one file. If the study requires several test runs, it is advisable to start data collection a reasonable distance upstream of the start point and to stop data collection once the test car passes the end point. For the next test run, the test car returns to the start point and the operator starts new data collection. The operator should also write down the comments to 
record the cause of delay, traffic conditions and road conditions. Therefore, one data file is created for each test run and no confusion will be generated for post-processing. It is also very common to perform data collection during the peak hours. For some special studies, the study time has been discussed in the ITE Manual.

Table 5.3 Format of Raw Data File

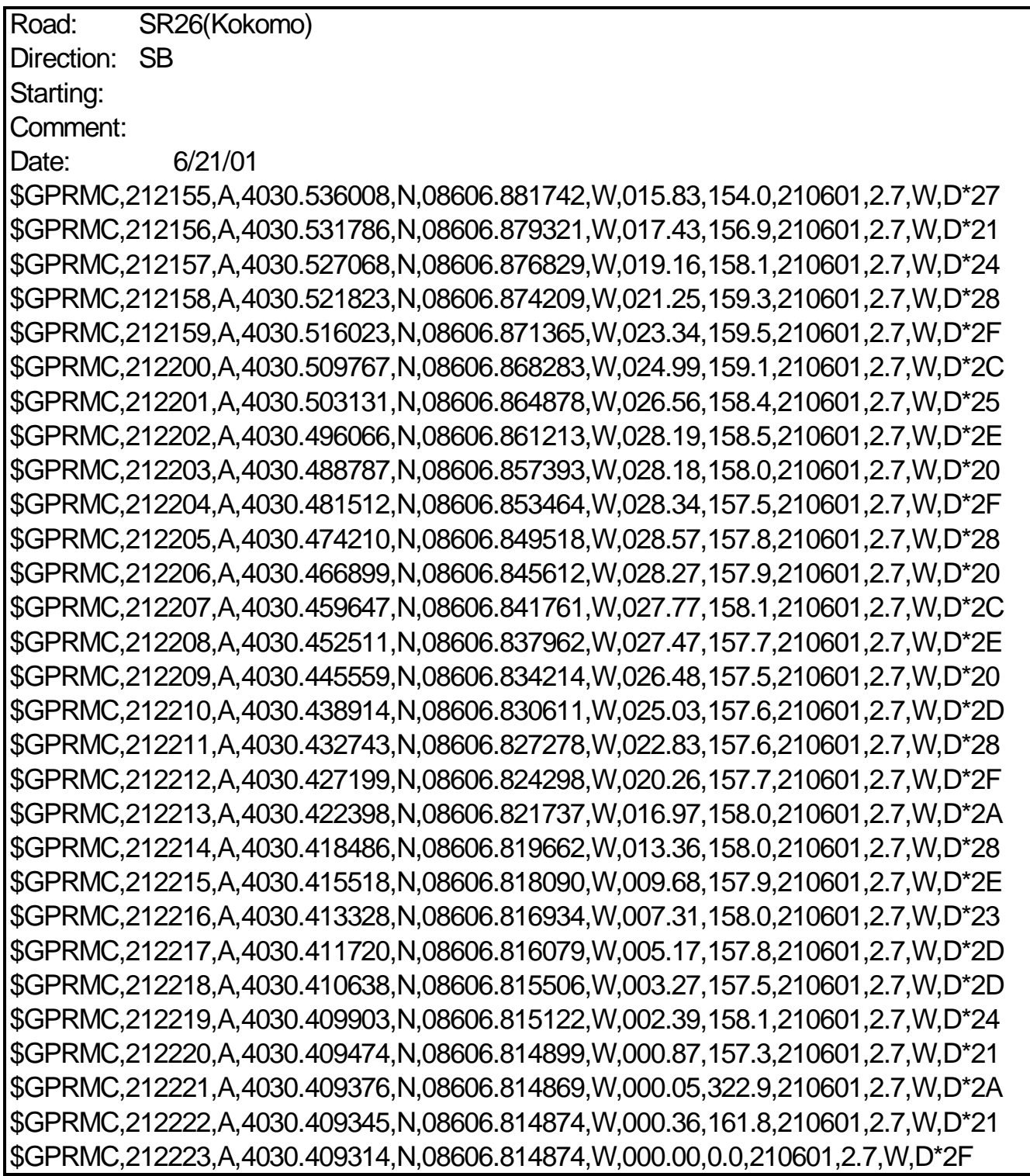


Chapter 6

\section{USE OF GPS-TREK IN TRANSPORTATION ENGINEERING STUDIES}

It is demonstrated that in the preceding chapters, there are many advantages to employ the GPS-Trek, the GPS-based data collection and processing program developed in this study for field transportation studies. Because the GPS-Trek can provide accurate and reliable basic traffic data, such as test car position and travel time, it can be readily utilized for (but not limited to) the most common transportation studies, including traveltime and delay studies, intersection stopped studies and congestion assessment. Also, the application of this system may be easily extended to some special transportation studies, such as work zone studies, car-following verification and roadway geometric design verification, because the GPS-Trek can generate an accurate trajectory or time-space diagram of the test car from the GPS position and time data. This chapter focuses on use of the basic GPS data collected using the GPS-Trek for travel-time and delay studies and intersection studies. Application to congestion assessment issues is also discussed. For other studies, the reader can refer to the related publications $(9,10)$. It is displayed that in the course of performing these field studies, the GPS-Trek can save manpower, improve efficiency of data collection and enhance field operation safety.

\subsection{Format of Fundamental Data File and Creation of Data Worksheet}

\section{Format of Fundamental Data File}

Similar to the current methods that are widely used for transportation engineering studies, the GPS-Trek measures the position of test car and records the corresponding travel time and creates a raw data file as discussed in the fourth chapter (or see Table 5.3). The raw data file can be converted into a new data file with an extension name, .gps. It should be kept in mind that if possible, the raw data should be processed as soon as the operator finishes the data collection. To avoid possible confusion that may be caused when we need to re-examine those data in the future, it is recommended that a specific 
file name be assigned to each data file and all comments made during data collection be kept properly.

As discussed in chapter 4 , the format of the data file after post-process, *.gps, is specially designed so that the data analysis can be carried out efficiently. Table 6.1 is an example of the format of the *.gps file. There are ten fields. The first five fields record the fundamental GPS data and the last five fields the basic traffic data and other information, such as the position of intersections and marks. The test date and test time (Indiana local) are saved in fields 1 and 2. The test car positional data, such as $\mathrm{X}$ and $\mathrm{Y}$ coordinates, are saved in fields 3 and 4. Field 5 contains the local UTM zone number. Travel distance is recorded in field 6. Travel speed is saved in filed 7. The eighth 8 field contains travel time that is computed from the test time saved in field 1 . Field 9 is used to record the positions of the intersections of interest. The last field is used to mark the special locations on the test route during data collection.

\section{Creation of Data Worksheets}

The fundamental data file as illustrated in Table 6.1 is an ASCII file and can be readily used to create a Microsoft ${ }^{\circledR}$ Excel worksheet. There are several advantages to use Microsoft ${ }^{\circledR}$ Excel to perform data analysis over other software. First, most transportation

engineers have sufficient knowledge about how to use Microsoft ${ }^{\circledR}$ Excel and no training is needed. Secondly, transportation engineers, such as district traffic engineers, may have certain special needs. Use of Microsoft ${ }^{\circledR}$ Excel allows transportation engineers to perform simple calculations so as to generate additional information based on their own professional experience. Fox example, transportation engineers can compute the average travel speed between intersections according to the data such as travel time, travel distance and intersection positions, and record the results in a new field. Table 6.2 is an example that shows how to add a new field, Average Speed that can be easily computed on the basis of travel time and travel distance at the intersections marked with the Arabic numeral, 0 . 
Table 6.1 Fundamental Data Worksheet

\begin{tabular}{|c|c|c|c|c|c|c|c|c|c|}
\hline \multirow{5}{*}{\begin{tabular}{|l} 
Road: \\
Direction: \\
Starting: \\
Comment: \\
Date: \\
Date (mmddyy)
\end{tabular}} & \multicolumn{9}{|l|}{ SR26 } \\
\hline & \multirow{2}{*}{\multicolumn{9}{|c|}{ Kokomo }} \\
\hline & & & & & & & & & \\
\hline & \multicolumn{9}{|l|}{ 6/21/01 } \\
\hline & Time & Xutm(m) & Yutm(m) & UTM Zone & Travel Dis (m) & Travel Speed (mph) & Travel Time(s) & Intersection & Mark \\
\hline 6/21/01 & 154733 & 575609.5 & 4479640.0 & 16 & 211.4 & 31.4 & 0 & & \\
\hline 6/21/01 & 154734 & 575608.3 & 4479626.5 & 16 & 224.9 & 29.2 & 1 & & \\
\hline $6 / 21 / 01$ & 154735 & 575607.2 & 4479614.0 & 16 & 237.5 & 27.1 & 2 & & \\
\hline $6 / 21 / 01$ & 154736 & 575606.0 & 4479602.4 & 16 & 249.1 & 25 & 3 & & \\
\hline $6 / 21 / 01$ & 154737 & 575605.0 & 4479591.8 & 16 & 259.8 & 22.9 & 4 & & \\
\hline $6 / 21 / 01$ & 154738 & 575604.0 & 4479581.9 & 16 & 269.8 & 21.7 & 5 & & \\
\hline $6 / 21 / 01$ & 154739 & 575602.9 & 4479572.4 & 16 & 279.3 & 20.9 & 6 & & \\
\hline $6 / 21 / 01$ & 154740 & 575601.9 & 4479563.3 & 16 & 288.5 & 20.3 & 7 & & \\
\hline $6 / 21 / 01$ & 154741 & 575600.8 & 4479554.4 & 16 & 297.4 & 19.7 & 8 & & \\
\hline $6 / 21 / 01$ & 154742 & 575599.7 & 4479545.9 & 16 & 306 & 18.8 & 9 & & \\
\hline $6 / 21 / 01$ & 154743 & 575598.6 & 4479537.9 & 16 & 314.1 & 17.4 & 10 & & \\
\hline $6 / 21 / 01$ & 154744 & 575597.7 & 4479530.7 & 16 & 321.4 & 15 & 11 & & \\
\hline $6 / 21 / 01$ & 154745 & 575596.9 & 4479524.7 & 16 & 327.4 & 11.9 & 12 & & \\
\hline $6 / 21 / 01$ & 154746 & 575596.3 & 4479520.3 & 16 & 331.8 & 8 & 13 & & \\
\hline $6 / 21 / 01$ & 154747 & 575595.9 & 4479517.6 & 16 & 334.6 & 4.5 & 14 & & \\
\hline $6 / 21 / 01$ & 154748 & 575595.7 & 4479516.3 & 16 & 335.9 & 1.3 & 15 & & \\
\hline $6 / 21 / 01$ & 154749 & 575595.7 & 4479516.1 & 16 & 336.1 & 0.1 & 16 & & \\
\hline $6 / 21 / 01$ & 154750 & 575595.7 & 4479516.1 & 16 & 336.2 & 0 & 17 & 0 & \\
\hline $6 / 21 / 01$ & 154751 & 575595.7 & 4479516.1 & 16 & 336.2 & 0 & 18 & & \\
\hline
\end{tabular}


Table 6.2 Example of a New Field with Additional Special Data

\begin{tabular}{|c|c|c|c|c|c|}
\hline Travel Dis. (f) & Travel Speed (mph) & Travel Time (s) & Intersection & Mark & Average Speed (mph) \\
\hline 21117.6 & 43.4 & 315 & & & 46.4 \\
\hline 21181.7 & 44.0 & 316 & & & 46.4 \\
\hline 21246.7 & 44.5 & 317 & 0 & & 46.4 \\
\hline 21311.9 & 44.4 & 318 & & & 43.1 \\
\hline 21376.5 & 43.8 & 319 & & & 43.1 \\
\hline 21440.1 & 43.0 & 320 & & & 43.1 \\
\hline 21502.5 & 42.1 & 321 & & & 43.1 \\
\hline 21563.4 & 41.0 & 322 & & & 43.1 \\
\hline 21623.9 & 41.4 & 323 & & & 43.1 \\
\hline 21685.0 & 42.0 & 324 & & & 43.1 \\
\hline 21747.1 & 42.8 & 325 & & & 43.1 \\
\hline 21810.3 & 43.4 & 326 & & & 43.1 \\
\hline 21874.1 & 43.5 & 327 & & & 43.1 \\
\hline 21937.9 & 43.4 & 328 & & & 43.1 \\
\hline 22001.1 & 42.8 & 329 & & & 43.1 \\
\hline 22063.6 & 42.4 & 330 & & & 43.1 \\
\hline 22125.8 & 42.5 & 331 & & & 43.1 \\
\hline 22188.3 & 42.8 & 332 & & & 43.1 \\
\hline 22251.4 & 43.2 & 333 & & & 43.1 \\
\hline 22315.1 & 43.6 & 334 & & & 43.1 \\
\hline 22379.2 & 43.9 & 335 & & & 43.1 \\
\hline 22443.9 & 44.4 & 336 & & & 43.1 \\
\hline 22509.3 & 44.8 & 337 & & & 43.1 \\
\hline 22574.7 & 44.3 & 338 & & & 43.1 \\
\hline 22639.2 & 43.6 & 339 & & & 43.1 \\
\hline 22702.4 & 42.7 & 340 & & & 43.1 \\
\hline 22764.5 & 41.9 & 341 & & & 43.1 \\
\hline 22825.2 & 40.9 & 342 & & & 43.1 \\
\hline 22885.5 & 41.4 & 343 & & & 43.1 \\
\hline 22946.8 & 42.1 & 344 & & & 43.1 \\
\hline 23009.1 & 42.9 & 345 & & & 43.1 \\
\hline 23072.5 & 43.5 & 346 & & & 43.1 \\
\hline 23136.8 & 44.1 & 347 & & & 43.1 \\
\hline 23202.0 & 44.8 & 348 & & & 43.1 \\
\hline 23267.6 & 44.7 & 349 & 0 & & 43.1 \\
\hline 23333.3 & 44.8 & 350 & & & 39.0 \\
\hline 23399.0 & 44.8 & 351 & & & 39.0 \\
\hline 23464.5 & 44.5 & 352 & & & 39.0 \\
\hline 23529.0 & 43.4 & 353 & & & 39.0 \\
\hline
\end{tabular}

As illustrated in Tables 6.1 and 6.2, the Arabic numeral 0 is selected to mark the intersection position. While any symbol or number can be used to mark the intersection position, it is believed that the Arabic numeral 0 is probably the best choice. This is because for transportation engineering studies, graphical reports may contain two different types of information (or variables), numerical numbers such as travel time, speed and distance and character strings such as intersection names. When Microsoft ${ }^{\circledR}$ 
Excel is employed to create charts, it can not label the axes or the data values in a chart using both numerical number and character string. For example, while creating a trajectory with the travel distance as the $\mathrm{x}$-axis, Microsoft ${ }^{\circledR}$ Excel can not show the intersection name. Although we can use any symbol to mark the intersection positions in the worksheet, use of the Arabic numeral 0 makes it easier to mark the intersection positions on the $\mathrm{x}$-axis in the chart and eliminates the potential problems during generating graphical reports. Obviously, this data file provides the basic data and it is pretty easy to add more fields to record additional information, such as average travel speed between intersections.

\subsection{Selection of Study Routes}

This project selected four study routes, US31 in Kokomo, SR26 in Lafayette, US52 in West Lafayette, and I65 in Indianapolis. Strictly speaking, selection of study route depends only on the purpose of study and the GPS-Trek has no any special requirements if GPS view is not limited while collecting field GPS data. The GPS-Trek is developed for general use in transportation studies and can be used for data collection in any places with good visibility. As discussed in Chapter 3, GPS devices may not provide continuous positioning in places such as urban areas surrounded by tall buildings and rural areas surrounded by forest. Therefore, this project selected study routes where GPS signals would not be blocked. To demonstrate the potential applications of the GPSTrek system, this project also took into account route length, traffic volume and type of signal systems while selecting the study routes.

\section{$\underline{\text { US31 in Kokomo }}$}

The test segment on US31 in Kokomo is about 9.5-miles long $(15 \mathrm{~km})$ and located in Kokomo as shown in Figure 6.1. One of the major purposes to select this route is to investigate use of the GPS-Trek in evaluation of the performance of the coordinated signal systems. This test route is a four-lane highway located in one vehicle manufacturing area and is one of the busiest routes in Indiana. The traffic volume is about 1,300 vph (one direction) and the post speed is $45 \mathrm{mph}$. There are fifteen intersections on 
the test segment and all signals at these fifteen intersections are coordinated. Two field studies were conducted on this test segment. The first field study was performed during off-peak hours on June 20, 2000 and the test car was traveling from SR26 to US31 so as to cover all signals. The second field study was conducted during peak-hours on June 15, 2001 and the test car was traveling from Morgan Street to Center Road, covering only twelve intersections. Both studies were to measure travel time and speed in southbound direction.

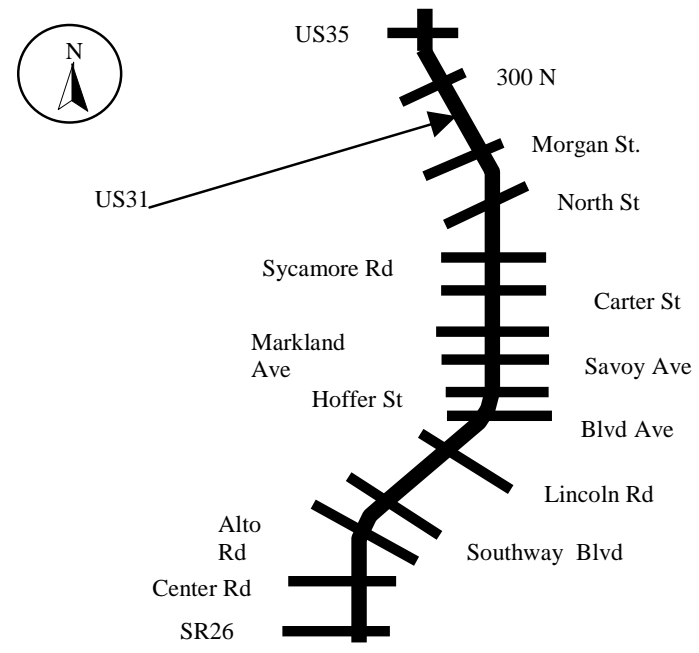

Figure 6.1 Graphical Illustration of Test Segment on US31 in Kokomo

\section{SR26 in Downtown Lafayette}

The second test route is on SR26 in downtown Lafayette and is located between US52 and I65. The test segment is about 1.8 mile $(2.9 \mathrm{~km})$ long, consisting of 10 coordinated signals. All the roads intersecting with SR26 are minor roads except for Crease Lane that is a four-lane street. This test segment has four lanes with a post speed of $40 \mathrm{mph}$ and the traffic volume is 1,200 vph (Southbound). Figure 6.2 shows the test segment and the physical positions of the signals. It should be pointed out that this figure is obtained using GPS data in June 2000 and does not contain the new signal that was recently installed between Farington Avenue and Wellspring Drive. Field data collection was conducted using the GPS-Trek. This test route mainly serves as a site for verifying sample size requirements and 12 test runs were completed within a one-hour period. 


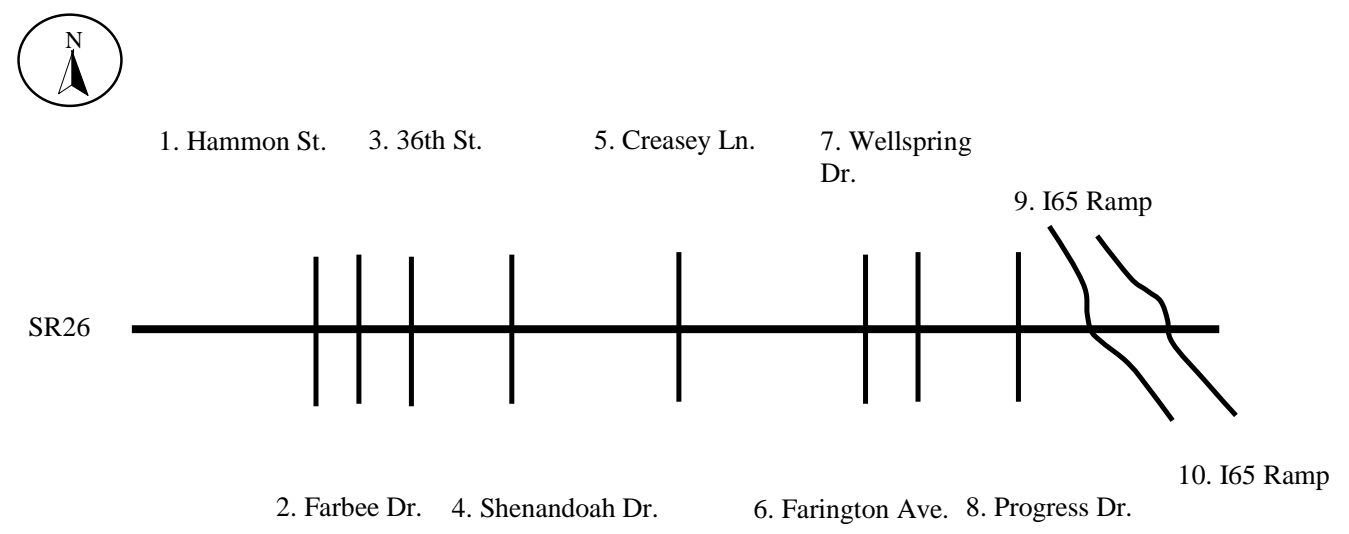

Figure 6.2 Graphical Illustration of Test Segment on SR26 in Lafayette

\section{US52 in West Lafayette}

The test route on US52 in Lafayette is the shortest test route selected by this study as shown in Figure 6.3. It is a four-lane highway with three signals. These three signals are not coordinated. All the three side roads are two-lane local roads. The distance between Yeager Road and Nighthawk Drive is about 0.7 miles $(1.1 \mathrm{~km})$. The traffic volume approaching the intersection with Yeager Road was about $700 \mathrm{vph}$ in eastbound on the test route and grew to $820 \mathrm{vph}$ over the segment between Yeager Road and Salisbury Street.

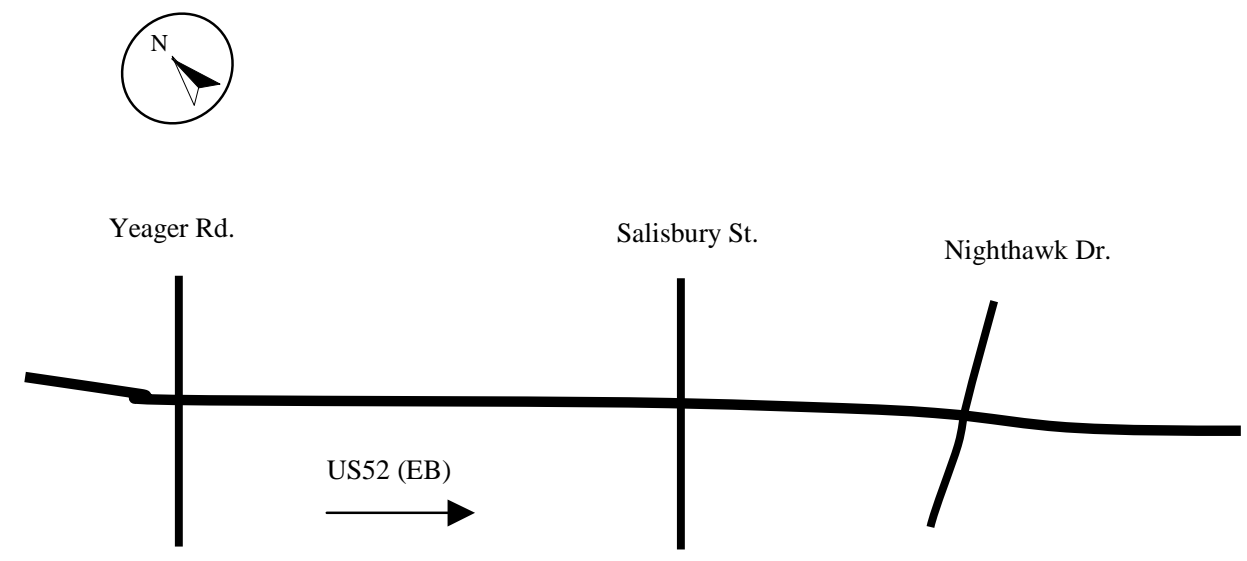

Figure 6.3 Graphical Illustration of Test Segment on SR26 in Lafayette 


\section{$\underline{\text { I65 in Indianapolis }}$}

The test route on I65 is located within a work zone in Indianapolis as shown in Figure 6.4. It is about 5 miles $(8 \mathrm{~km})$ long. This work zone is a mixed work zone with a partial closure layout at some locations and a crossover layout at other locations. There are two major purposes to select a test segment with a work zone on freeway. The first purpose is to evaluate the safety while operating the GPS-Trek at high traveling speed. The second purpose is to further investigate use of the GPS-Trek in work zone studies. Because Figure 6.4 is created on the basis of actual GPS position data using ArcView ${ }^{\circledR}$ GIS (or Microsoft ${ }^{\circledR}$ Excel), it shows the physical position of the test route and provides us with the real geometrical feature of the route within the work zone. As a result, we can better understand the acceleration and deceleration of the vehicles traveling within the work zone.

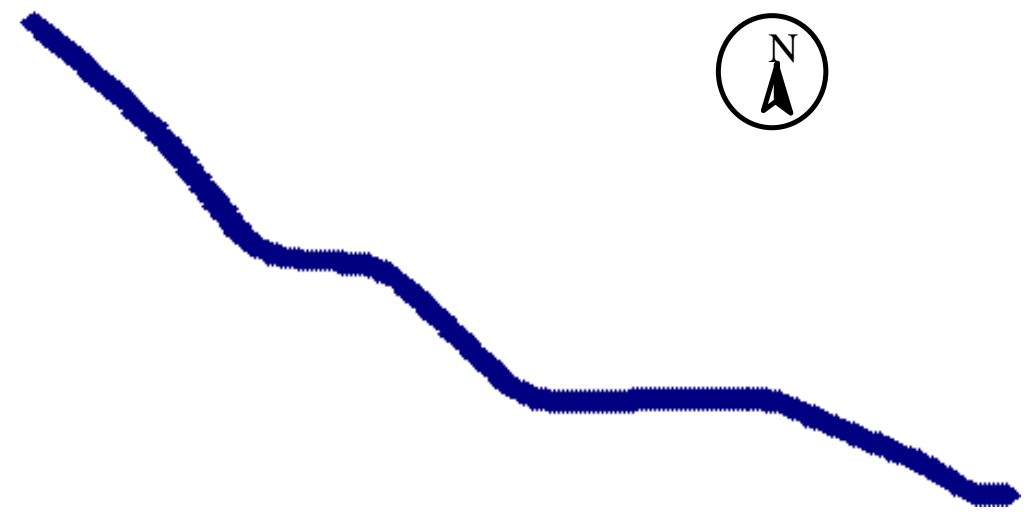

Figure 6.4 Graphical Illustration of Test Segment on I65 in Indianapolis

\subsection{Application to Travel Time Studies}

\section{Measurements of Travel Time and Distance}

To investigate operation of a vehicle, transportation engineers need to track the positions of the vehicle over time. This is usually done on the basis of a time-space diagram or a trajectory of the vehicle. Travel time can be directly obtained from the worksheet as illustrated in Tables 6.1 and 6.2 and expressed as 
$\mathrm{T}=\mathrm{t}_{\mathrm{i}+1}-\mathrm{t}_{\mathrm{i}}$

where $\mathrm{T}$ is the travel time between points $\mathrm{i}$ and $(\mathrm{i}+1)$, and $\mathrm{t}$ is the corresponding time measurement in field 8 in Table 6.1.

Travel distance between any two points is calculated using the following equation

$\mathrm{D}=\mathrm{d}_{\mathrm{i}+1}-\mathrm{d}_{\mathrm{i}}$

where $\mathrm{D}$ is the travel distance between points $\mathrm{i}$ and (i+1), and $\mathrm{d}$ is the corresponding distance measurement in field 6 in Table 6.1.

Once the travel time and distance data are available, we can create a vehicle trajectory. For example, we can use the Microsoft ${ }^{\circledR}$ Excel ChartWizard to visualize such the trajectory by displaying the data points on field 6 and 8 in Table 6.1. Because the GPS-Trek can mark all critical points as instructed by the user, the created vehicle trajectory will present the physical positions of the marked critical points, such as intersections and start point or end point in a work zone, on the x-axis accurately. As a result, it is possible for us to provide graphical reports that are recommended in the Manual of Transportation Engineering Studies and get a full picture about how the test car travels over the test route.

\section{Case Studies}

Figure 6.5 demonstrates two vehicle trajectories created on the basis of GPS data measured on US31 in Kokomo. The first trajectory (Figure 6.5a) is created using the data measured at 10:00 a.m. on June 20, 2000, covering the whole test route and all signals (see Figure 6.1). The second trajectory (Figure 6.5b) is based on the data measured at 16:30 p.m. on June 15, 2001, running from Morgan Street to Center Road. It is shown that the travel distance is about $15 \mathrm{~km}$ (9.4 miles) between US31 and SR26 and $9 \mathrm{~km}(5.6$ miles) between Morgan Street and Center Road. The corresponding travel time is about 810 seconds and 790 seconds, respectively. 


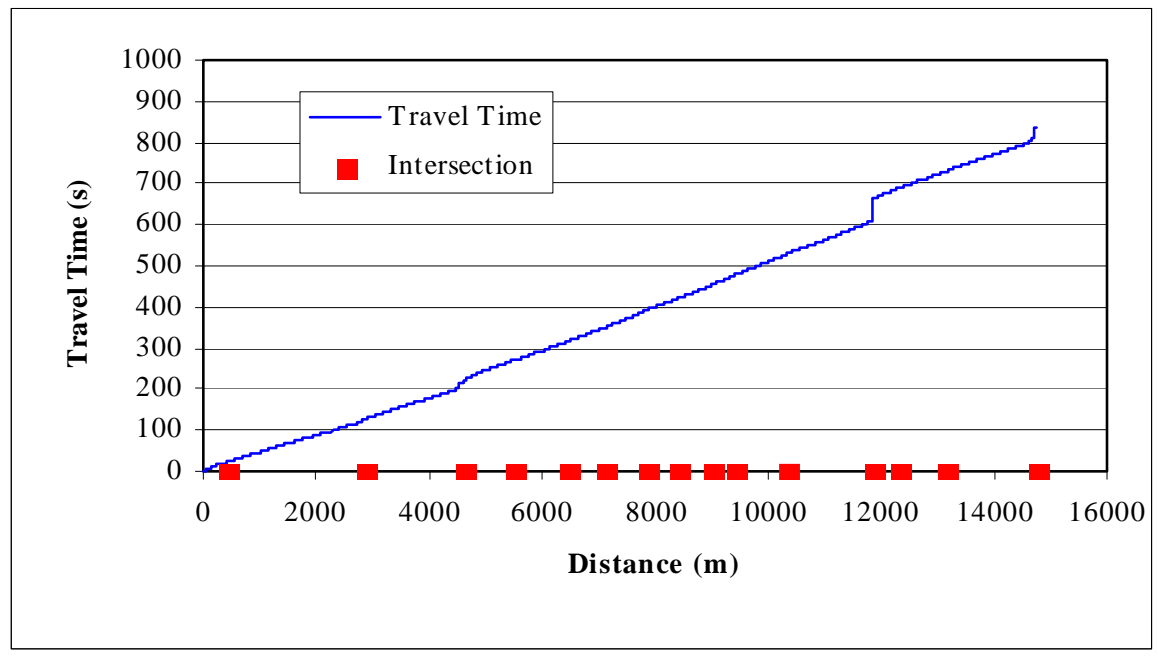

(a) Trajectory Measured on June 20, 2000 (10:00 a.m.)

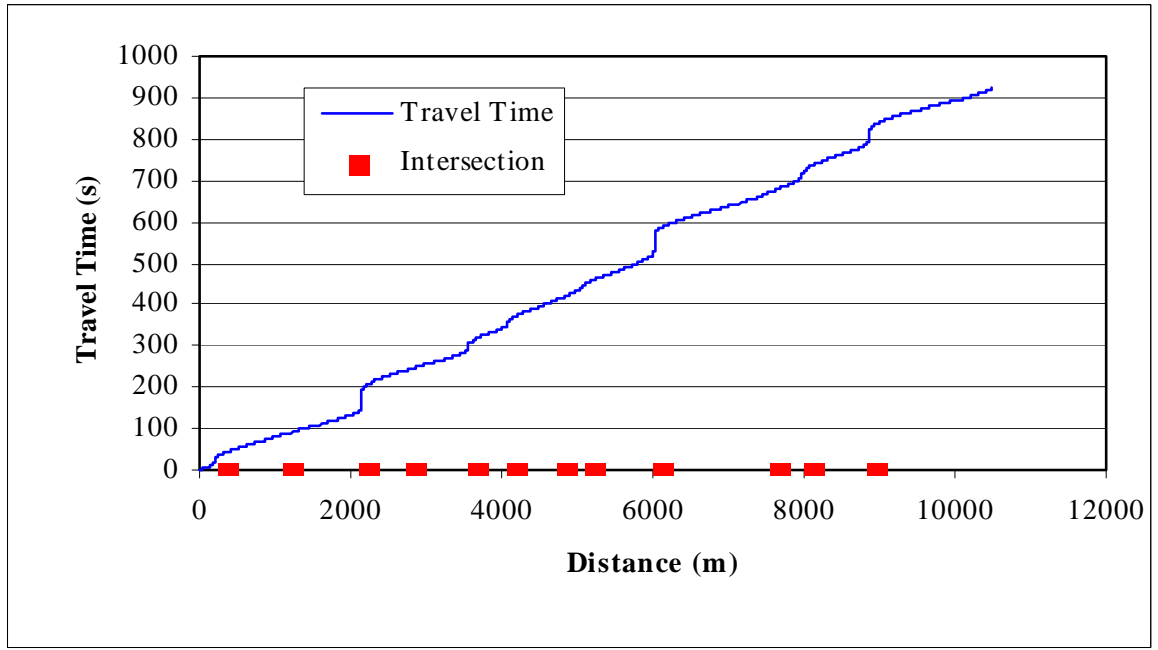

(b) Trajectory Measured on June 15, 2001 (16:30 p.m.)

\section{Figure 6.5 Trajectories Measured Using GPS-Trek on US31 in Kokomo}

Careful inspection of the trajectories in Figure 6.5 indicates that in general, the test car traveled very steadily over the test segments. During the first test as shown in Figure 6.5 (a), the test car traveled almost at a constant speed and was stopped only at the intersection with Southway Boulevard. The second test was performed during the peakhours and the test car was stopped at three intersections with Sycamore Road, Lincoln Road and Center Road, respectively, resulting in increase in the travel time. The 
trajectory fluctuates because of the deceleration and acceleration of the test car due to the signals and increasing inaction with other vehicles.

Vehicle trajectories are created for the test routes on SR26 in Lafayette and on US52 in West Lafayette as shown in Figures 6.6 and 6.7, respectively. It is illustrated that once the test car was stopped at intersections and started to move again, it accelerated to a specific speed quickly and then traveled very steadily between intersections. It takes about 280 seconds for the test car to travel 3,000 meters over the test route on SR26, and 100 seconds to travel 1,100 meters between Yeager Road and Nighthawk Drive on US52. The test car was stopped for about 50 seconds at the intersection with Creasey Lane on SR26 and 20 seconds at the intersection with Salisbury Street on US52, which may be the major bottlenecks in the systems. Obviously, the GPS-Trek can be used to perform travel time studies. It can provide an accurate trajectory that presents us with an overview of traffic situation along the test route. Also, it should be highlighted that the vehicle trajectory created using the GPS-Trek may have many other applications. The accuracy and the reliability depend mainly on the data rate of the GPS receiver used in the study.

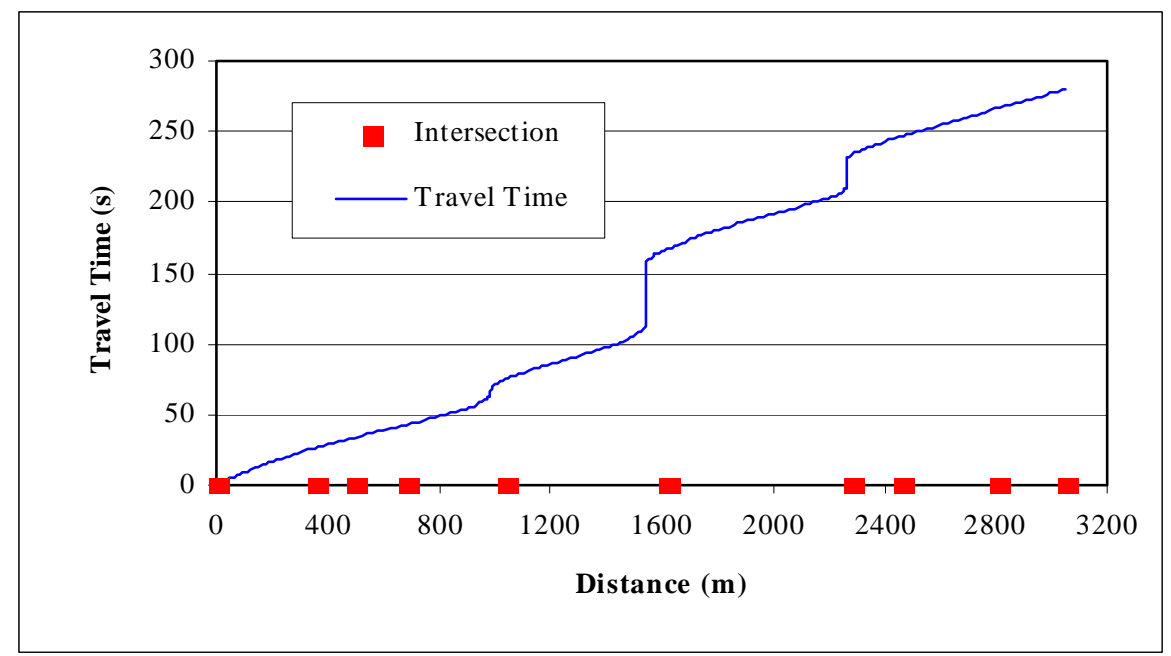

Figure 6.6 Trajectory Measured on SR26 in Lafayette (9:00 a.m.) 


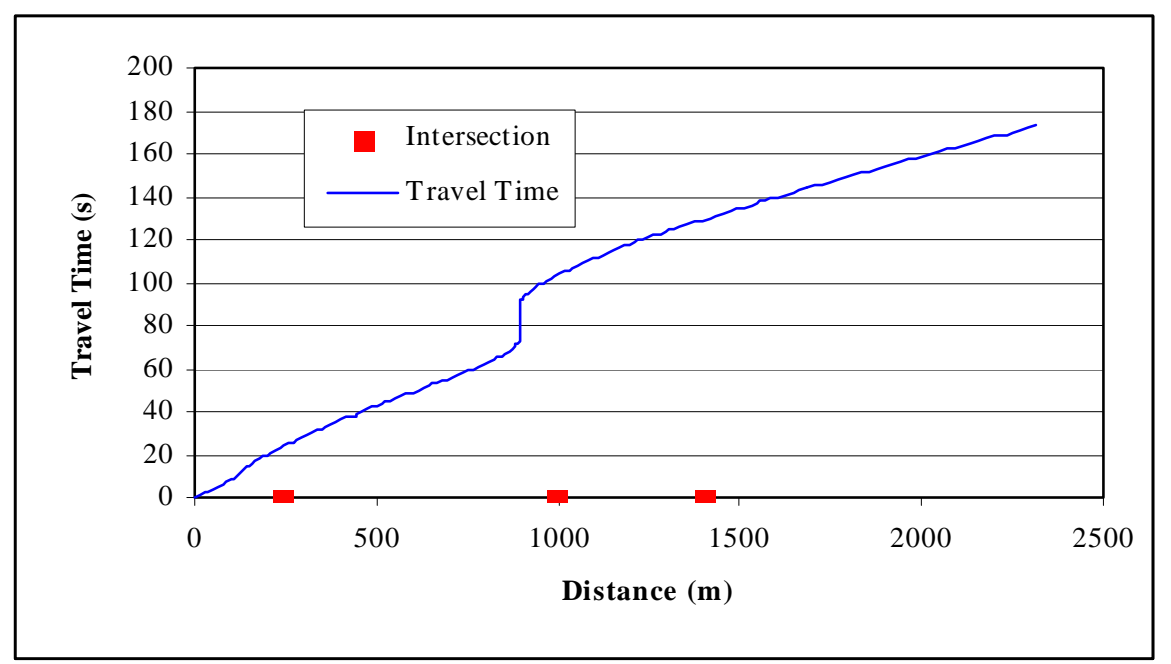

Figure 6.7 Trajectory Measured on US52 in West Lafayette (9:30 a.m.)

\subsection{Application to Travel Speed Studies}

\section{Measurement of Travel Speed}

Travel speed is the reciprocal of the travel time and is an important measure used in HCM (11) to evaluate levels of service for rural two-lane highways, arterial streets and freeway weaving sections. Because travel speed is a simple field measurement and easy for the public to understand, it has been widely used in economic analysis, safety study and convenience analysis. There are two types of average speed, time-mean speed and space-mean speed, which are measured using different devices and methods. With GPS devices, we may measure both time-mean and space-mean speeds at the same time.

To use a GPS device to measure spot speed, it is necessary for us to examine the fundamentals of speed computation employed in the GPS device. As discussed in the previous chapters, a GPS receiver outputs real time position data in terms of latitude and longitude. For most GPS devices, however, they can also output the so-called velocity. This velocity is not a direct speed measurement by the GPS device and is actually a calculated speed using the following equation 
$\mathrm{V}=\frac{\sqrt{\left(\mathrm{x}_{2}-\mathrm{x}_{1}\right)^{2}+\left(\mathrm{y}_{1}-\mathrm{y}_{2}\right)^{2}}}{\Delta \mathrm{t}}$

where $\mathrm{V}$ is the velocity between two consecutive points 1 and 2 , $\mathrm{x}$ and $\mathrm{y}$ are the position coordinates of points 1 and 2 , and $\Delta \mathrm{t}$ is the time interval between these two points. For a GPS device, $\Delta \mathrm{t}$ is referred to as the data rate.

It is shown that the velocity obtained using Eq. 6.3 is equivalent to the indirect measurement of speed measured using vehicle detectors such as inductive loops. When two inductive loops are used to measure speed, they are spaced a specific distance apart and the time for the vehicle to travel from the first loop to the next loop is measured. The distance between the two loops is called the speed trap. In reality, the measured speed using this technique is space speed. If the speed trap is very short, however, the measured speed can be considered as spot speed. It is noted that there exist differences between the speeds measured using vehicle detectors and GPS devices. First, the speed trap is not constant. It is actually the distance between the positions of the GPS device at two different times, i.e. $\sqrt{\left(\mathrm{x}_{2}-\mathrm{x}_{1}\right)^{2}+\left(\mathrm{y}_{1}-\mathrm{y}_{2}\right)^{2}}$, and changes from location to location and time to time. Secondly, the speed trap associated with the use of the GPS devices is much larger than the speed trap for vehicle detectors. As velocity grows, the speed trap increases. With a GPS data rate of $1 \mathrm{~Hz}$ (one output per second), the speed trap will vary from about 10 meters on a local road to 30 meters on a freeway. As a result, there exist discrepancies between the GPS velocity and the desired spot speed. For a test route of one mile or longer, a speed trap of 10 to 30 meters is still very short. If the road condition remains unchanged, the traffic flow will be steady and the GPS velocity will be close to the spot speed over such a short trap.

With GPS devices, however, we can measure space-mean speed that may be accurate enough for any transportation studies. As demonstrated in Tables 6.1 and 6.2, the GPS-Trek not only records the real time position data, but produces travel time and travel distance data as well. Based on these data, we can divide the whole test route into 
many sections and calculate the corresponding space-mean speeds using the equation below

$S=\frac{d_{i+1}-d_{i}}{t_{i+1}-t_{i}}$

where $\mathrm{S}$ is the space-mean speed, $\mathrm{d}$ is the travel distance and $\mathrm{t}$ is the travel time as shown in Table 6.2.

\section{Case Studies}

Travel speed data were also measured over the four test routes as described earlier. Based on the data worksheet created using the GPS-Trek, we can readily create two types of graphical reports on travel speed, i.e. the speed profile, and the average speed between those critical points along the test route. The speed profile allows us to examine the acceleration and deceleration characteristics of the vehicle in detail. It can be also used to address many other issues such as stop distance. The average speed provides us with a general view of the travel speed between intersections and is useful for timing traffic signals, especially coordinated signal systems. Figures 6.8, 6.9, 6.10 and 6.11 show the graphical summary of speed over the four test routes, respectively.

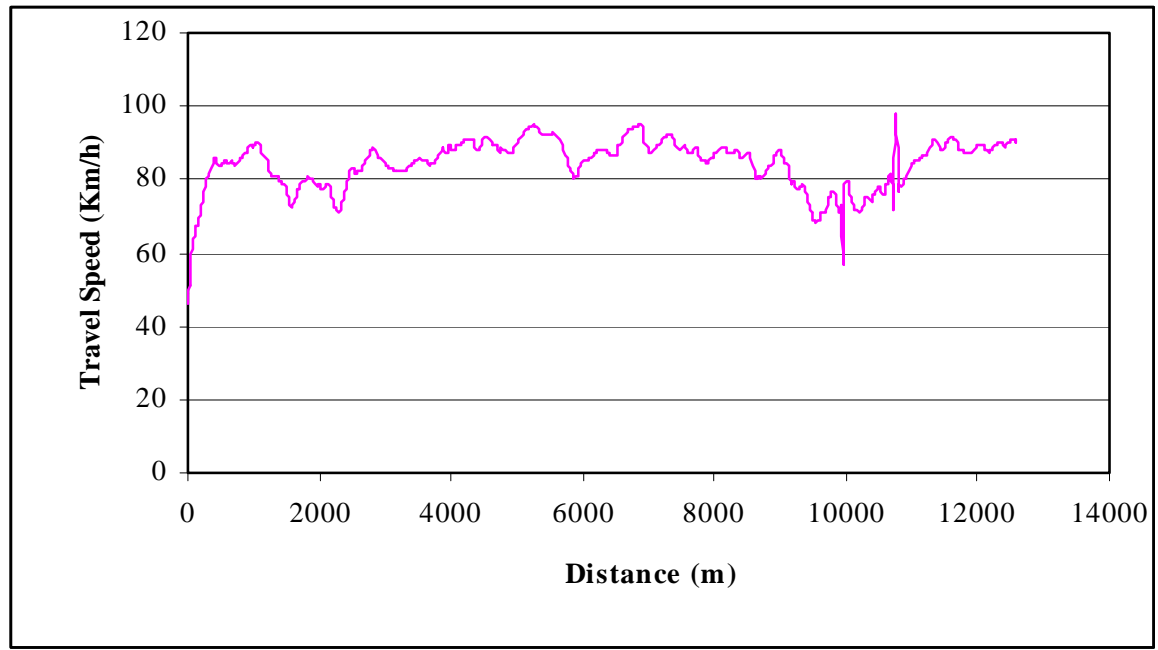

Figure 6.8 Speed Profile on I65 in Indianapolis (11:30 a.m.) 


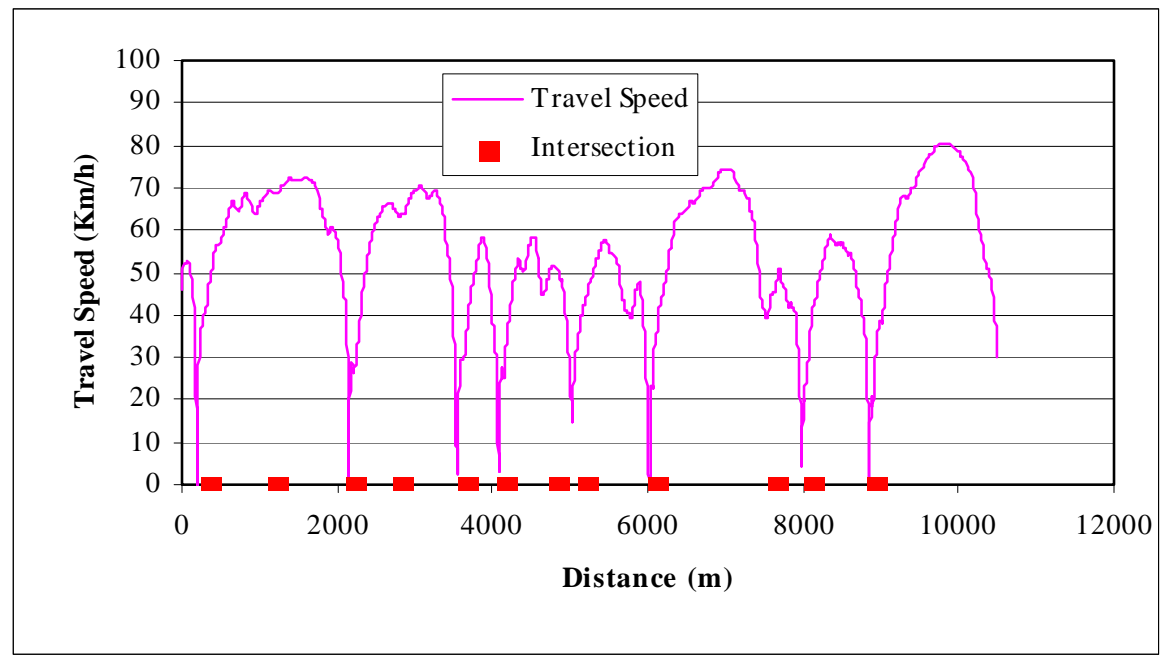

(a) Speed Profile

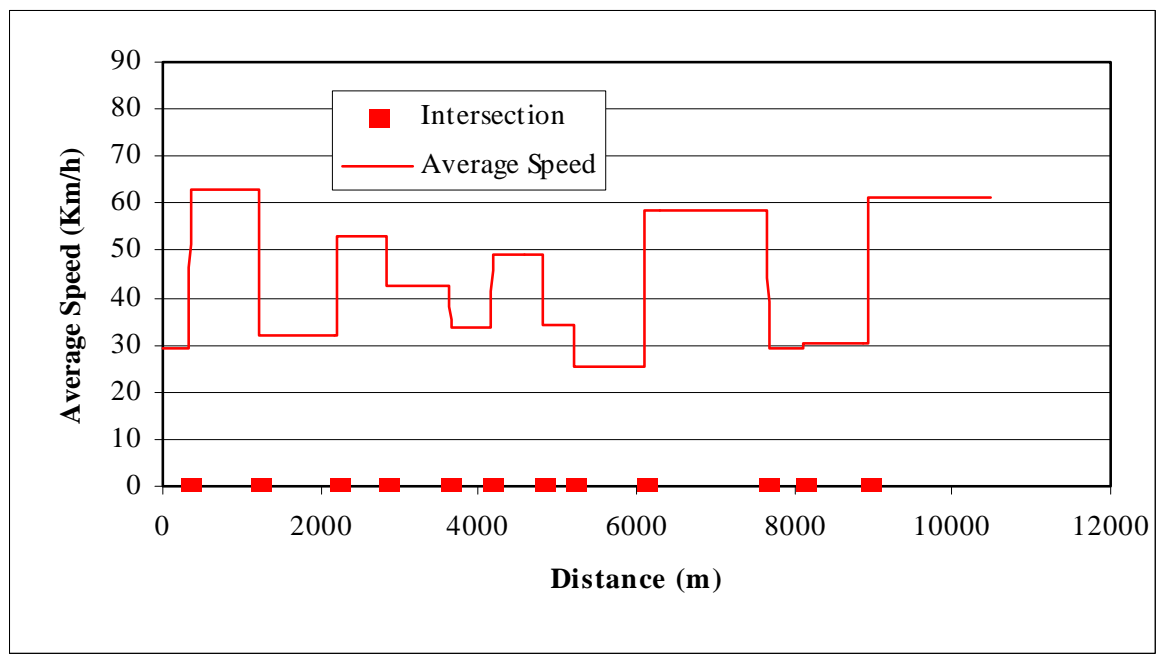

(b) Average Speed

Figure 6.9 Summary of Travel Speed on US31 (16:30 p.m.) 


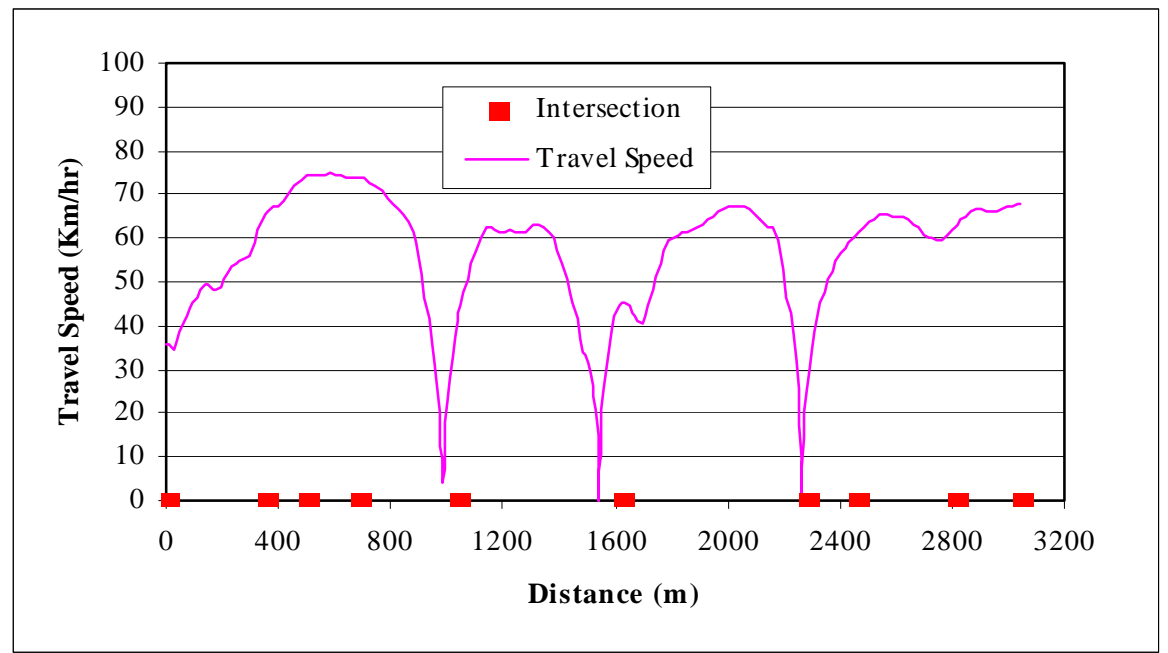

(a) Speed Profile

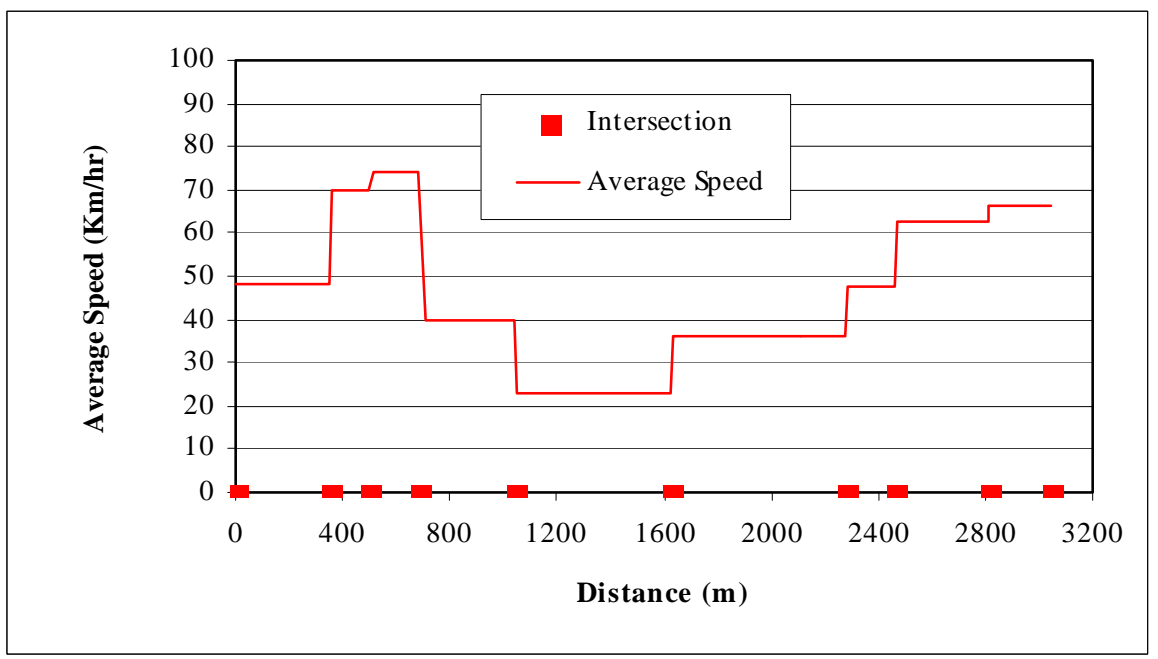

(b) Average Speed

Figure 6.10 Summary of Travel Speed on SR26 (9:00 a.m.) 


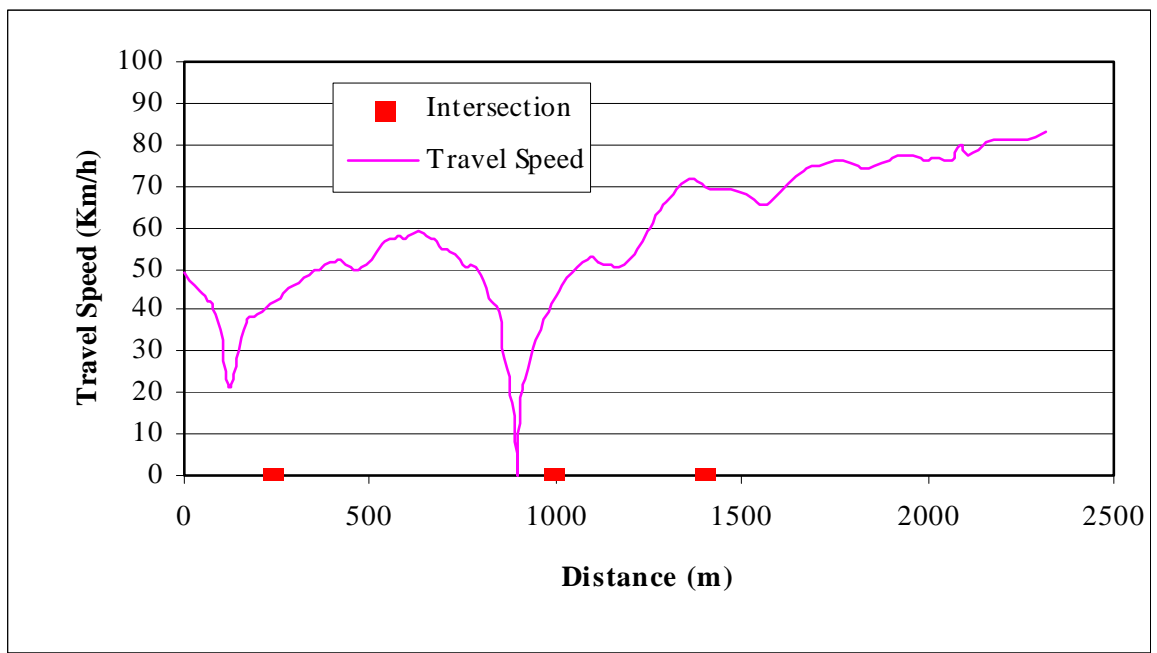

(a) Speed Profile

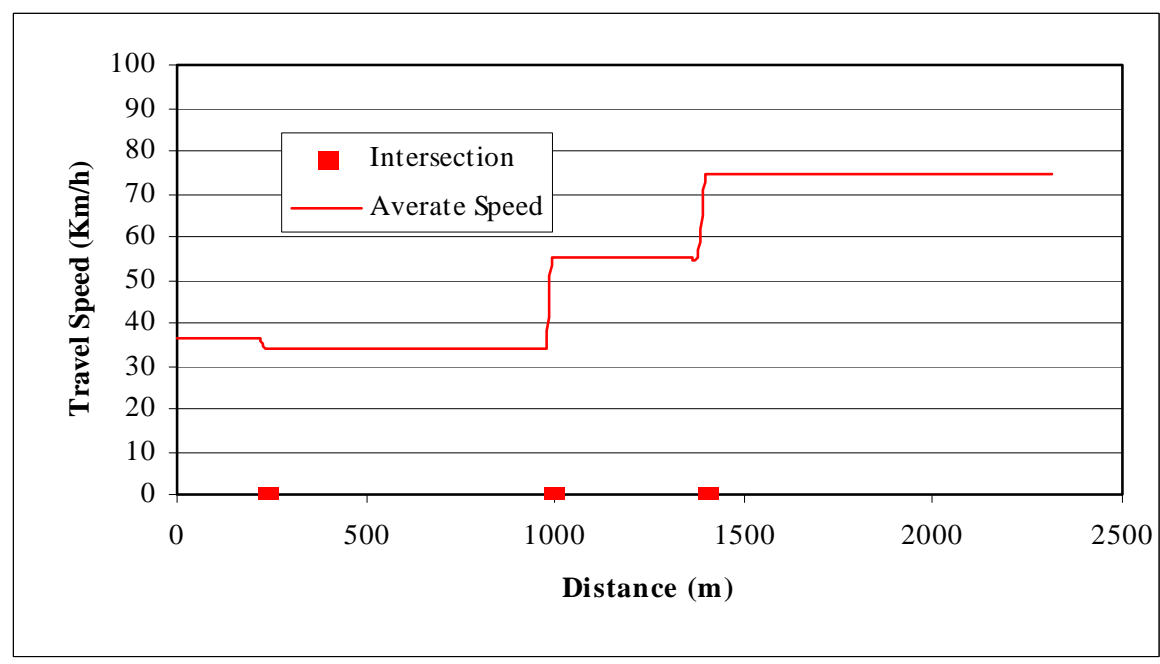

(b) Average Speed

Figure 6.11 Summary of Travel Speed on US52 (9:30 a.m.)

Figure 6.8 gives the speed profile on I65 within the work zone in Indianapolis. The test car accelerated to merge to I65 from the on-ramp. Then, the test car reduced its speed and traveled into the work zone. After the test car entered the work zone, it accelerated to a certain speed and maintained this speed within the work zone. Because of the effect arising in the weaving areas, the travel speed fluctuated as illustrated from 
10,000 to 11,000 meters. In Figures 6.9, 6.10 and 6.11, the speed profile shows clearly the driving maneuver between intersections and the positions where the test car was stopped. It is demonstrated that the test car usually increased its speed to pass one intersection. After the test car accelerated to a specific speed, it slowed down when approaching the next intersection. Because of the effect of signals, the test car experienced frequent acceleration and deceleration. The average speed varied considerably from intersection to intersection, depending on the performance of the signal system, the length between intersections and traffic volume. Based on the average speed, we can roughly know which parts in the system do perform well.

\subsection{Application to Travel Delay and Congestion Studies}

\section{Measurement of Travel Delay}

Travel delay has been widely used in conjunction with travel time by transportation engineers to measure performance of transportation facilities, such as freeways, highway corridors and signalized urban street systems. It is also used as an estimate of impact of improvements on highway facilities. There are two measurements of travel delay, stopped delay and total delay. The former is usually used to measure delay at individual intersection and the latter the delay over the whole system. For a highway system, such as a highway corridor, the stopped delay usually constitutes a major portion of the total delay and is often used to estimate the total delay.

\section{Stopped Delay}

Based on the definition of the stopped delay, we can estimate it from the vehicle trajectory (see Figures 6.5, 6.6 and 6.7) or quantify it from the data worksheet (see Table 6.2) as follows:

$$
\mathrm{T}_{\mathrm{SD}}=\sum_{\text {Speed }=0} \Delta \mathrm{t}
$$


where $\mathrm{T}_{\mathrm{SD}}$ is the stopped delay, $\Delta \mathrm{t}$ is the amount of time while the vehicle is idling and can be determined by examining the seventh field of Travel Speed in Table 6.1. If travel speed equals zero, the vehicle is stopped. If the test route consists of many signals, we can get a view of stopped delay over the whole system by inspecting the speed profile as illustrated in Figures 6.8, 6.9, 6.10 and 6.11.

\section{Total Delay}

While total delay may be estimated using some empirical equations with respect to the stopped delay, caution must be exercised when using those equations. With the GPS-Trek, however, we can measure both stopped delay and total delay simultaneously, resulting in a great saving in terms of manpower and time. The total delay can be computed as follows

$\mathrm{T}_{\mathrm{TD}}=\mathrm{T}_{\mathrm{t}}-\mathrm{T}_{0}$

where $\mathrm{T}_{\mathrm{TD}}$ is the total delay, $\mathrm{T}_{\mathrm{t}}$ is the actual travel time, and $\mathrm{T}_{0}$ is the estimated travel time without delay.

The actual travel time can be determined from the data worksheet according to the positions of the start and end points of the test route. The estimated travel time without delay can be evaluated as follows

$\mathrm{T}_{0}=\frac{\mathrm{D}}{\mathrm{V}_{\mathrm{a}}}$

where $\mathrm{D}$ is the distance of the test route and $\mathrm{V}_{\mathrm{a}}$ is the approach speed which can be determined from the speed profile. In some cases, such as urban streets in downtown, it is very difficult to find the approach speed from the speed profile because of the surroundings, and $\mathrm{V}_{\mathrm{a}}$ can be assumed to be the ideal speed or simply the post speed. 
To illustrate the procedures to compute the total delay based on the data worksheet created using the GPS-Trek, we can refer to the speed profile as shown in Figure 6.12, which was obtained from the data measured in the work zone on I65. The test car merged onto I65 from the on-ramp and then accelerated to a specific speed while approaching the work zone. After entering the work zone, the test car reduced its speed. The test car accelerated again after passing the work zone. The total travel time $\left(\mathrm{T}_{\mathrm{t}}\right)$ is 470 seconds, and the length of the work zone is 11,387.2 meters. It is indicated that the approach speed $\left(\mathrm{V}_{\mathrm{a}}\right)$ is about $100 \mathrm{~km} / \mathrm{h}$ or $27.71 \mathrm{~m} / \mathrm{s}$. Therefore, the estimated travel time without delay is

$$
\mathrm{T}_{0}=\frac{\mathrm{D}}{\mathrm{V}_{\mathrm{a}}}=\frac{11387.2 \mathrm{~m}}{27.71 \mathrm{~m} / \mathrm{s}}=411 \text { seconds }
$$

The total delay is

$$
\mathrm{T}_{\mathrm{TD}}=\mathrm{T}_{\mathrm{t}}-\mathrm{T}_{0}=470-411=59 \text { seconds }
$$

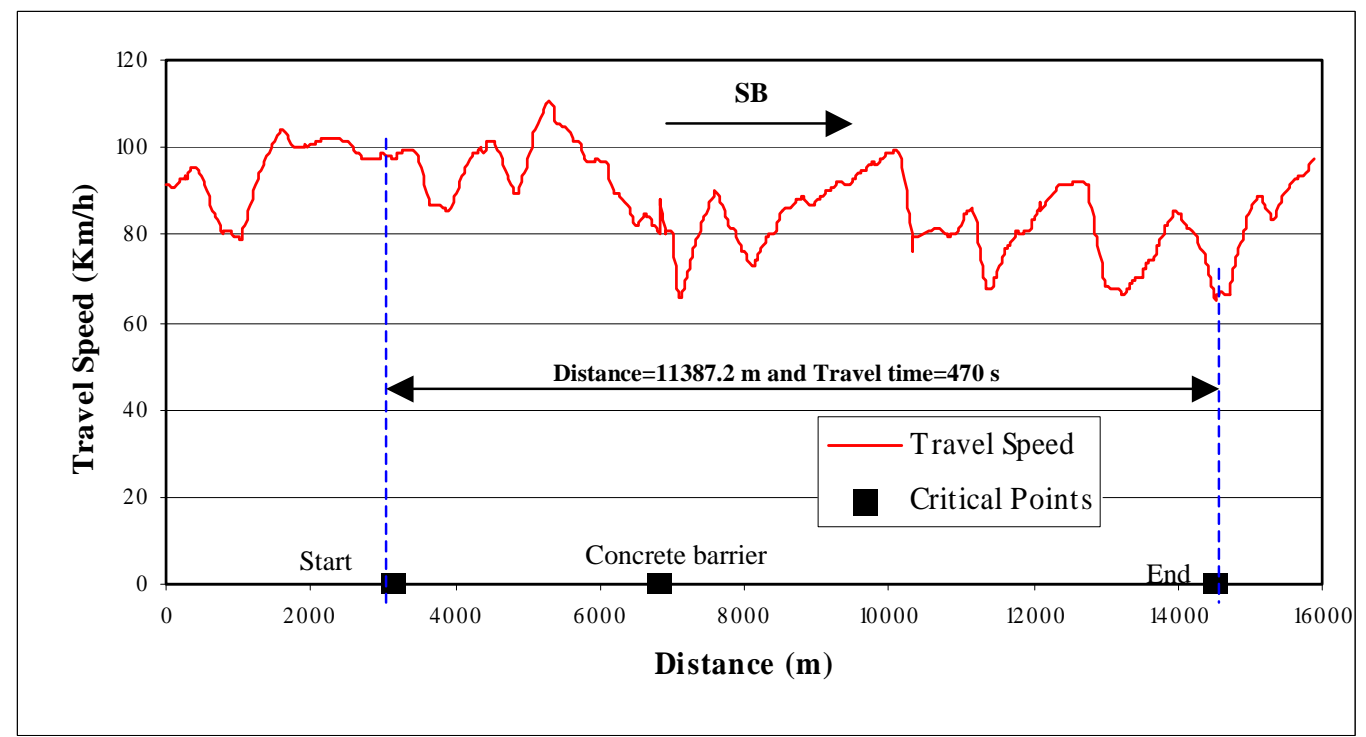

Figure 6.12 Speed Profile in the Work Zone on I65 
The above procedure can be also used evaluate delay for signal systems. Figure 6.13 shows a speed profile measured on US31 in Kokomo. The test section started at the intersection with Lincoln Road and ended at Sycamore Road. The measured travel distance is about 3,888.2 meters, travel time is bout 310 seconds and stopped delay is 88 seconds. It is estimated from the speed profile that the approach speed is about $80 \mathrm{~km} / \mathrm{h}$ $(22.2 \mathrm{~m} / \mathrm{s})$. The travel time without delay should be

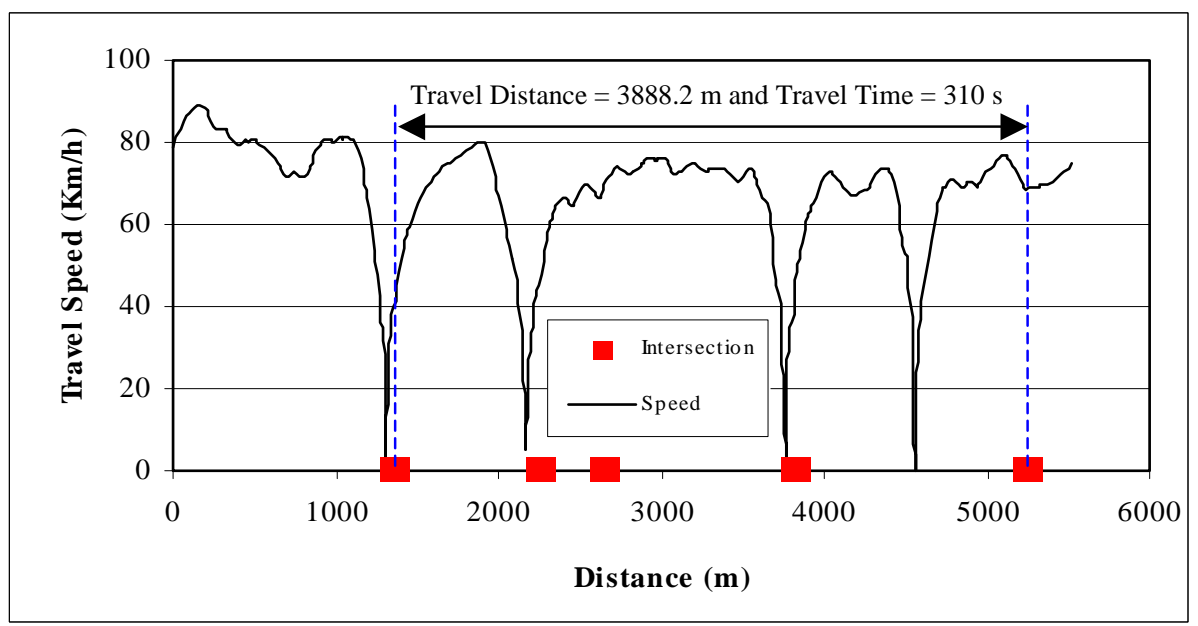

Figure 6.13 Speed Profile on US31

$$
\mathrm{T}_{0}=\frac{\mathrm{D}}{\mathrm{V}_{\mathrm{a}}}=\frac{3888.2 \mathrm{~m}}{22.2 \mathrm{~m} / \mathrm{s}}=175 \text { seconds }
$$

The total delay is

$$
\mathrm{T}_{\mathrm{TD}}=\mathrm{T}_{\mathrm{t}}-\mathrm{T}_{0}=310-175=135 \text { seconds }
$$

It is illustrated that the stopped delay is 88 seconds and the estimated total delay is 135 seconds. The difference is 47 seconds. Therefore, the stopped delay accounts for about $65 \%$ of the total delay and the remainder are the time losses due to the acceleration and deceleration of the test car and the effect of other vehicles. Obviously, these results are essential for evaluating signal performance. 


\section{Measurement of Congestion}

Congestion is an additional travel time beyond that usually experienced by a motorist under light or free flow travel conditions. While travel time and delay are the foundation for measuring congestion, the concept of congestion has been widely used to evaluate the existing traffic conditions in cities, metropolitan areas, freeways and work zones. Tim et al. examined the state of the practice in congestion measurement and identified the basic congestion measures (12). It is demonstrated that in the preceding sections that the GPS-Trek can provide reliable travel time and delay data for evaluating coordinated signal systems, identifying bottlenecks along a roadway and estimating travel situation with work zones. Moreover, the data worksheet established using the GPS-Trek contains detailed travel information and can be used to develop travel time, travel delay and travel speed database. Thus, the GPS-Trek can be readily utilized to address congestion issues on the basis of travel time, speed and delay.

Travel rate is calculated with respect to the average speed. This is a basic congestion measure to evaluate the rate of motion over a specific route and has many uses, especially for individual roadways. The definition of travel rate is given below (12)

Travel Rate $=\frac{\text { Travel Time }}{\text { Segment Length }}=\frac{60}{\text { Average Speed }}$

where the travel time is measured in minutes, segment length in miles, average speed in $\mathrm{mph}$, and the travel rate is measured in minutes per mile ( 1 mile $=1.609$ kilometers).

Delay rate is another basic congestion measure for assessing the rate of time loss while traveling under congested conditions and has been employed to ranking congested facilities and prioritize alternative improvements. It is defined as the time difference between the actual travel time and the acceptable travel time per mile and can be computed using the following equations (12) 
Delay Rate $=\frac{\text { Actual Travel Time }- \text { Acceptable Travel Time }}{\text { Segment Length }}$

where the travel time is measured in minutes, segment length in miles and delay rate in minutes per mile.

It is shown that in Table 6.2 that the worksheet created using the GPS-Trek contains all information essential for computing both the travel rate and the delay rate. Table 6.3 is an example that shows the calculation of the travel rate and delay rate. The results were obtained on the basis of the worksheet for US31 in Kokomo. The acceptable travel time is solely determined using the travel speed without delay, i.e. the approach speed $(80 \mathrm{~km} / \mathrm{h})$. It is presented that the segment from Boulevard Avenue to Lincoln has the greatest travel rate and delay rate. The delay rate is obtained by reducing the travel rate and both measures provide similar results. Figure 6.14 gives a graphical illustration of the travel and delay rates. It is apparent that both the travel rate and the delay rate follow the similar trend. This implies that while prioritizing alternatives, the travel rate and the delay rate can produce the same results.

Table 6.3 Travel Rate and Delay Rate for US31 in Kokomo

\begin{tabular}{|l|c|c|c|c|c|c|}
\hline \multirow{2}{*}{ Intersection } & $\begin{array}{c}\text { Segment } \\
\text { Length }\end{array}$ & $\begin{array}{c}\text { Average } \\
\text { Speed }\end{array}$ & Travel Time & Travel Rate & $\begin{array}{c}\text { Acceptable } \\
\text { Travel Time }\end{array}$ & Delay Rate \\
\cline { 2 - 7 } & $(\mathbf{m i l e s})$ & $(\mathbf{m p h})$ & (minutes) & (min/mile) & (minutes) & (min/mile) \\
\hline Morgan St & & & & & & \\
\hline North St & 0.54 & 39.1 & 0.82 & 1.5 & 0.64 & 0.3 \\
\hline Sycamore Rd & 0.62 & 19.8 & 1.88 & 3.0 & 0.75 & 1.8 \\
\hline Carter St & 0.38 & 32.8 & 0.70 & 1.8 & 0.46 & 0.6 \\
\hline Markland Ave & 0.50 & 26.4 & 1.13 & 2.3 & 0.60 & 1.1 \\
\hline Savoy Ave & 0.33 & 21.0 & 0.93 & 2.8 & 0.40 & 1.6 \\
\hline Hoffer St & 0.40 & 30.4 & 0.78 & 2.0 & 0.48 & 0.8 \\
\hline Blvd. Ave & 0.24 & 21.3 & 0.68 & 2.8 & 0.29 & 1.6 \\
\hline Lincoln Rd & 0.56 & 15.8 & 2.10 & 3.8 & 0.67 & 2.6 \\
\hline Southway Blvd & 0.97 & 36.4 & 1.58 & 1.6 & 1.16 & 0.4 \\
\hline Alto Rd & 0.28 & 18.1 & 0.93 & 3.3 & 0.34 & 2.1 \\
\hline Center Rd & 0.50 & 19.0 & 1.58 & 3.1 & 0.60 & 1.9 \\
\hline
\end{tabular}

$(1 \mathrm{mile}=1.609 \mathrm{~km})$ 


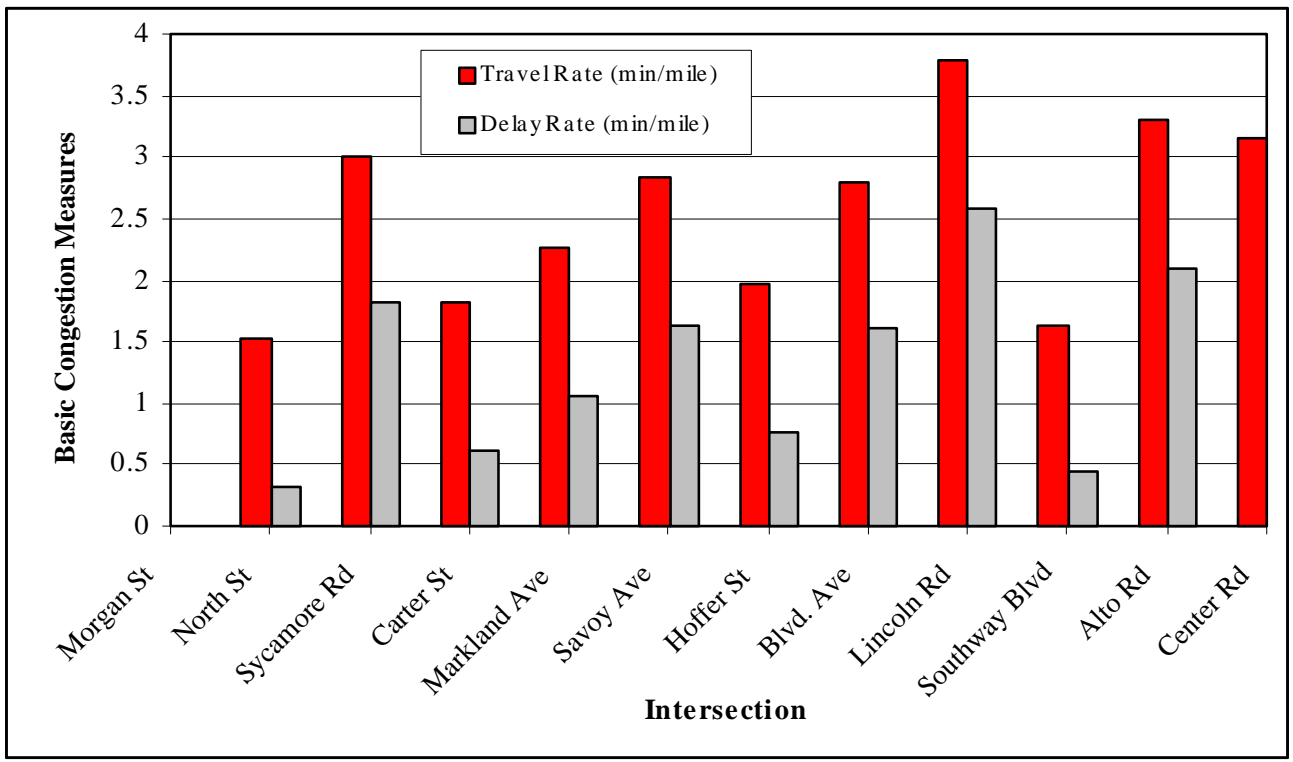

Figure 6.14 Illustrations of Travel and Delay Rates on US31 in Kokomo

\section{$\underline{\text { Assessing Congestion at Management Level }}$}

For traffic engineers at management level, they may need to assess congestion in a highway or street system monthly, quarterly or yearly. Because a highway system is multi-dimensional and geographically broad, it is necessary to develop a way to equip traffic engineers to carry out this work more efficiently. As discussed earlier, we can use travel rate or delay rate to measure congestion over an individual road. However, it may not be convenient to use the two rates to measure congestion in a highway system. This is because the travel rate or the delay rate requires measuring travel time and route length between two locations and there is no a unique reference point in a regional highway system. A possible way is to utilize a speed diagram to roughly assess congestion situation in the system because of the following reasons:

- Speed is the reciprocal of the travel time and is easy for the public to understand

- Speed can be directly measured using GPS devices and does not require additional information and reference point, and 
- Traffic engineers can create a speed diagram without tedious calculation and visualize the speed variations in the whole system based only on the data file obtained using the GPS-Trek

The speed diagram may consist mainly of a street system map and the measured speed information. The speed measurements can be classified into various groups and displayed in the speed diagram using different colors. Based on such a diagram, traffic engineers can get a brief view of the traffic condition over a roadway system. Traffic engineers may create various speed diagrams at different times so as to examine the variations of traffic condition speed with time at specific locations or over specific routes. Traffic engineers may also create speed diagrams at the same time on various days or in different seasons and then compare them to the before condition. As a result of these efforts, traffic engineers at the management level may identify the bottlenecks and the aspect of the congestion in the system.

Figure 6.15 shows two speed diagrams that were created for a selected street network in West Lafayette using the GPS-Trek. The street system consists of a state road (dark lines), i.e. US52 that runs through the central area, and local streets (blue lines). Also, there are three uncoordinated signals over US52 in the system. The first speed diagram was created during the off-peak hour period (around 10:30 am) and the second within the peak-hour period (around 12:30 pm). The test car started at the INDOT Research Division building (white dot), traveled over local streets and then over US52. As an illustration, the speed measurements were divided into two groups using a valve value of $25 \mathrm{mph}(40 \mathrm{~km} / \mathrm{h})$. The red line indicates those speed values lower than $25 \mathrm{mph}$, and the green line the speed values equal to or greater than $25 \mathrm{mph}$. It is shown that the traffic conditions over local streets are similar at the two different test times and the test car only slowed down when making a turn. Over US52, the test car traveled at a speed greater than $25 \mathrm{mph}$ and only slowed down when approaching one traffic signal around 10:30 am. At 12:30 pm, however, the test car slowed down when approaching two signals and traveled at a speed lower than $25 \mathrm{mph}$ even in two segments between the intersections. 


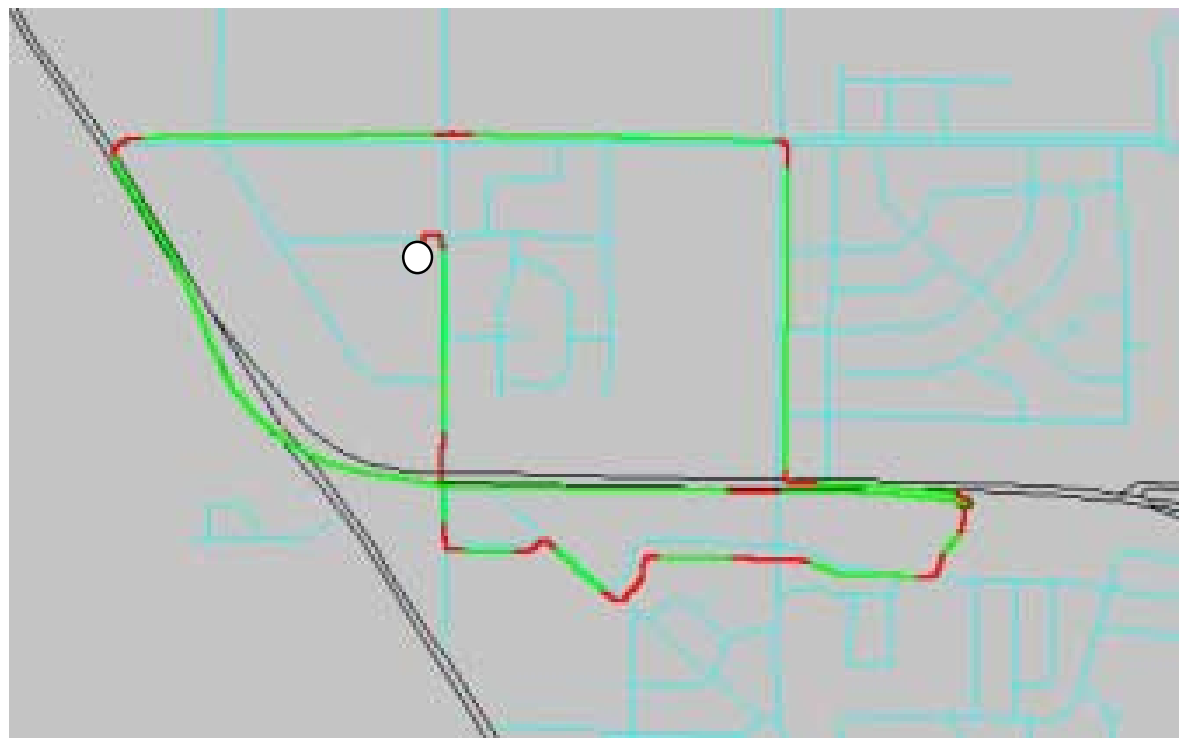

(a) Speed Diagram Measured at 10:30 am

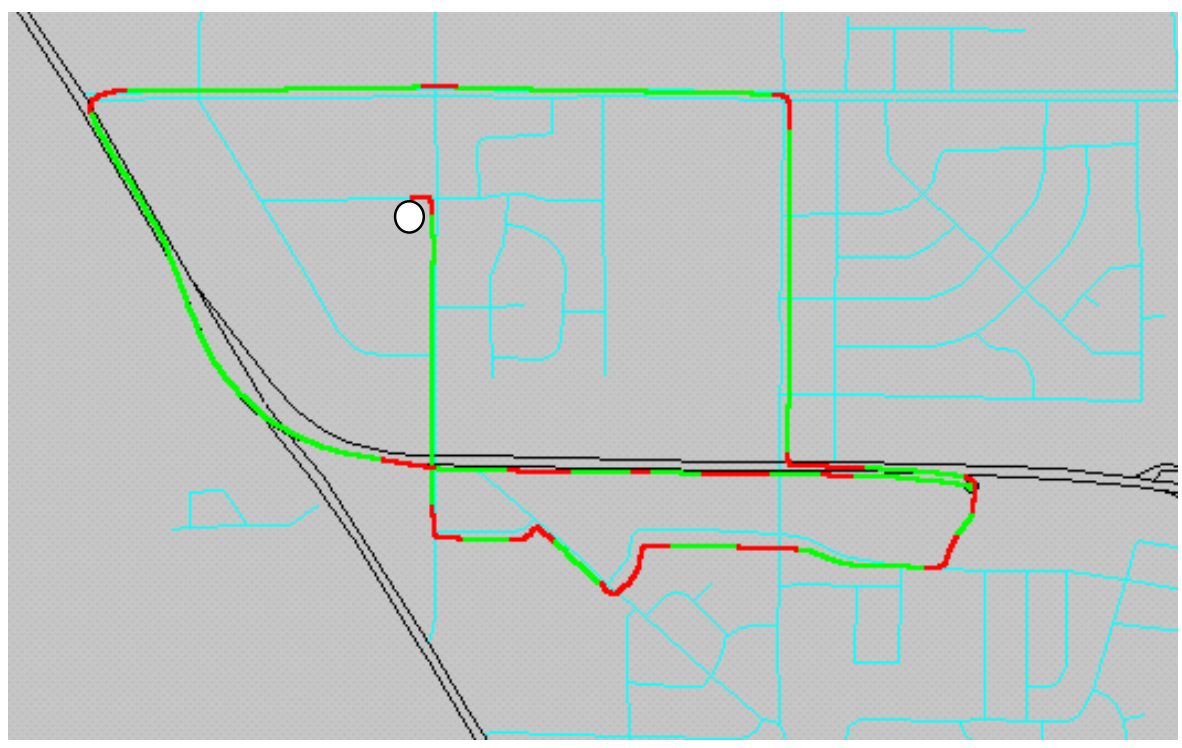

(b) Speed Diagram Measured at 12:30 pm

Figure 6.15 Speed Diagrams Created in West Lafayette 


\section{Chapter 7}

\section{CONCLUSIONS, RECOMMENDATIONS AND IMPLEMENTATION}

In this study, the GPS technique was examined, and one of the latest GPS devices was tested and validated using the precisely known geographic points. A procedure was derived to collect, store, process and manage GPS data and create new data files or worksheets for transportation studies. A GPS data collection system, consisting mainly of $\operatorname{AgGPS}^{\mathrm{TM}}$ 132, the GPS-Trek program and a laptop computer was established for traffic data collection and processing. A huge amount of data was collected at selected sites so as to verify the GPS data collection system, to evaluate the variations of the data collected using the GPS devices and to estimate sample size requirements for transportation studies. This study also investigated use of the GPS-Trek program to create worksheets for data analysis and investigated their applications to transportation studies, such as travel time and delay studies and congestion measurements.

\subsection{Conclusions}

Based on the work accomplished in this feasibility study, a brief summary of main conclusions is presented below:

The proposed system for traffic data collection using devices based on the positioning with the help of satellites of the Global Positioning System (GPS) is an inexpensive and cost-effective new alternative for classical analog methods of data gathering. An $\operatorname{AgGPS}^{\mathrm{TM}} 132$ receiver/antenna capable of operating in real-time differential mode costs about $\$ 5,000$. The annual fee for a DGPS service is $\$ 800$ per year. The data collection and processing program, GPS-Trek, enables traffic engineers to acquire and process field traffic data more efficiently and safely. In most cases, this system requires only one operator for data collection. The system was designed to 
minimize operator intervention during data collection, so as to reduce distraction and enhance operation safety.

The proposed system provides traffic data of high accuracy and reliability. At a data rate of $1 \mathrm{~Hz}$, the $\operatorname{AgGPS}^{\mathrm{TM}} 132$ can provide positional data with high and consistent quality for transportation studies. The field positional data may exhibit gaps, especially in "deep" urban canyons. However, these disadvantages may be successfully limited through interpolation and proper data screening. The removal of Selective Availability (SA) from the GPS signals by US Government (as of May 1, 2000) during the course of this investigation further improved the relative positional accuracy. It was shown that in a recent test, the AgGPS ${ }^{\mathrm{TM}} 132$ with DGPS function provided a positional accuracy well below the 0.5 meter. The absolute accuracy seemed to have become very competitive: the root mean square error (r.m.s.) of the stand alone mode (SA off) and the differential mode (SA off, DGPS on) seem to be of the same 1 meter level.

The GIS portion of the GPS-Trek program utilizes the existing INDOT highway system base map. This eliminates the need for creating new base maps and results in a great saving in terms of money, time and manpower. The GPS-Trek program consists of two components, one for data collection and the other for data processing, which can be run independently. This program can be utilized for data collection, storage, conversion and management. The data files created using the GPS-Trek program are reusable and their application is versatile. They can be readily used for transportation studies, development of database for congestion analysis and model calibrations, and road mapping. Information is saved in a data format that takes minimal storage space. Data file can be easily converted and exported to Micrsoft ${ }^{\circledR}$ Excel. This allows traffic engineers to perform data analysis and transportation studies based on the realistic field data and their own experience and judgment. Functions are provided for the operator to mark critical positions and make comments while collecting data or during data processing. The graphical user interface of the program is intuitive and user friendly so that minimal training is required to run the program. Data collection is free of personal interpretation. 
It provides a consistent system of analysis, especially attractive in decentralized operations, e.g. in the INDOT districts.

Determination of sample size depends on the variations of data and is also a tradeoff between the required accuracy and the cost. It requires knowledge of data dispersion, i.e. the standard deviation. Use of any estimate of the standard deviation, such as range or average range will generate errors in the process of determining the minimum sample size. The range only considers the two extreme values instead of all values and is the least satisfactory of all measures of dispersion. As a result, those methods based on the range will overestimate the minimum sample size. While the current ITE method uses the average range, it gives greater weights to the intermediate values in the process of computing the average range. This usually results in underestimating the minimum sample size. A modified equation for a confidence level of 90\%, 95\% and $99 \%$ was presented to estimate the minimum sample size without tedious calculation. It is shown that in the filed tests, the modified equation can produce a realistic estimate of the minimum sample size. To determine the sample sizes for most transportation studies, a minimum of three initial test runs should be considered. If possible, five initial test runs for $90 \%$ confidence or six for $95 \%$ confidence are recommended so as to reduce the potential significant errors.

An appropriate field procedure is required for GPS traffic data collection. It is of great importance that before driving to the test road, the operator should install the GPS and external devices properly, rather than on site. Before installation, the operator should always follow the safety instructions for the GPS devices, laptop, power inverter and DC power supply, so as to reduce damage to the devices. In most cases, one operator can conduct field data collection safely and efficiently. However, two operators are recommended for some special studies that require marking positions or events in the process of data collection.

The system developed in this study can be readily used for various transportation studies. The GPS-Trek program allows us to mark any critical points so that the created 
vehicle trajectory can present the physical positions of the marked points, such as intersections and start point or end point in a work zone. As a result, it is possible for us to provide graphical reports that are recommended in the Manual of Transportation Engineering Studies and get a full picture about how the test car travels over the test route. Based on the data files created using the GPS-Trek program, we can perform travel time studies and establish an accurate trajectory with marked points. We can conduct travel speed studies and measure speed profile and average travel speed. We can determine both stopped delay and total delay in a precise manner. Moreover, we can measure congestion, identify bottlenecks in a highway system on a statewide basis or a district basis and prioritize alternatives of improvement.

\subsection{Implementation Recommendations}

Based on aforementioned conclusions, the SPR-2392 research group proposes that the GPS data collection developed in this study should be considered by INDOT as a standard method for traffic data collection and analysis. Trials conducted during this phase of the study reveal that the analysis efficiency at the management level will be greatly enhanced if a state-wide (electronic) traffic signals data base is being integrated as a layer under the existing base map. Trials performed during this feasibility study also show that the GPS positional data collected in this study can be utilized to improve the INDOT's digital based map and state route database. It is recommended that a future study reveal how to imbed the traffic positional data into the state route data base on a regular basis.

Workshops will be conducted to provide instruction to initialize and operate the system. Trainings will be provided for

- Installing GPS data collection system

- Operating AgGPS ${ }^{\mathrm{TM}} 132$ devices

- Running the GPS-Trek program

- Exporting traffic data files created using the GPS-Trek program for traffic studies, and 
- Brainstorming sessions to enhance existing traffic data analysis techniques based on the highly accurate and dense data sets

It is proposed that this feasibility study lead to possible implementation and new test trials to be conducted in one or more INDOT districts so as to address issues at the management level. Experience gathered during these trials will lead to improved and more detailed manuals of operation of the GPS data collection system. 


\section{REFERENCES}

(1) Gilbert Strang and Kai Borre. Linear Algebra, Geodesy, and GPS. WellesleyCambridge Press, Wellesley, 1997.

(2) Trimble Navigation Limited. AgGPS ${ }^{T M}$ 124/132 Operation Manual. Sunnyvale, 1999.

(3) Worldwide Digital Global Positioning Service: How It Works Technical Description. OmniSTAR USA, Inc. http://www.omnistar.com/how_techdesc.html. Accessed January 22, 2000.

(4) The White House Office of the Press Secretary. Statement by the President Regarding the United States’ Decision to Stop Degrading Global Positioning System Accuracy. May 1, 2000. http://www.ngs.noaa.gov/FGCS/info/sans_SA/docs/statement.html. Accessed May 17, 2000.

(5) Handbook for Transformation of Datums, Projections, Grids and Common Coordinate Systems. TEC-SR-7. US Army Corps of Engineers, Topographic Engineering Center, 1996.

(6) Manual of Transportation Engineering Studies. Institute of Transportation Engineers, Washington DC, 2000.

(7) Quiroga, C. A., and Darcy Bullock. Development of CMS Monitoring Procedures. Louisiana Transportation Research Center, Louisiana State University, 1998.

(8) Shuo Li. Reliability Theories and Algorithms in Road Engineering. Shaanxi Science and Technology Publishing, Xian, 1990.

(9) Yi Jiang and Shuo Li. Measuring and Analyzing Vehicle Position and Speed Data at Work Zones Using Global Positioning System. ITE Journal, March 2002, pp. 48-53.

(10) Wolshon, B., and Y. Hatipkarasulu. Results of Car Following Analyses Using Global Positioning System. Journal of Transportation Engineering, ASCE, Vol. 126, No. 4, 2000, pp. 324-331.

(11) Highway Capacity Manual (HCM). Special Report 209, $3^{\text {rd }}$ Edition, Transportation Research Board, National Research Council, Washington, D.C., 1998.

(12) Tim Lomax, Shawn Turner, Gordon Shunk, Herbert S. L., Richard H., Paul N. B., and Douglas, G. B. Quantifying Congestion, NCHRP Report 398, Volume 1, Final 
Report, Transportation Research Board, National Research Council, Washington, D.C., 1997. 